\title{
Biomimetic Total Synthesis of the Rubiginosin Meroterpenoids
}

Laura Burchill, Aaron J. Day, Oussama Yahiaoui, and Jonathan H. George

Department of Chemistry, University of Adelaide, Adelaide, SA 5005, Australia.

\section{Supporting Information}

\section{Table of Contents}

1. General Methods 2

2. Experimental Procedures 3

3. NMR Spectra 19

4. Comparison of Natural and Synthetic NMR Data 58 


\section{General Methods}

All chemicals were purchased from commercial suppliers and used as received. All reactions were performed under an inert atmosphere of $\mathrm{N}_{2}$. All organic extracts were dried over anhydrous magnesium sulfate. Thin layer chromatography was performed using aluminium sheets coated with silica gel $F_{254}$. Visualization was aided by viewing under a UV lamp and staining with ceric ammonium molybdate or $\mathrm{KMnO}_{4}$ stain followed by heating. All $\mathrm{R}_{\mathrm{f}}$ values were measured to the nearest 0.05 . Flash column chromatography was performed using 40-63 micron grade silica gel or using a Biotage ${ }^{\circledR}$ Isolera $^{\mathrm{TM}}$ One Flash Chromatography System with LOKTM Empty Solid Load Cartridges, a Carl Roth silica gel 60 (230 - 400) mesh grade) where specified. Melting points were recorded on a digital melting point apparatus and are uncorrected. Infrared spectra were recorded using an FT-IR spectrometer as the neat compounds. High field NMR spectra were recorded using either a $500 \mathrm{MHz}$ spectrometer $\left({ }^{1} \mathrm{H}\right.$ at 500 $\mathrm{MHz},{ }^{13} \mathrm{C}$ at $125 \mathrm{MHz}$ ) or $600 \mathrm{MHz}$ spectrometer $\left({ }^{1} \mathrm{H}\right.$ at $600 \mathrm{MHz},{ }^{13} \mathrm{C}$ at $\left.150 \mathrm{MHz}\right)$. The solvent used for NMR spectra was $\mathrm{CDCl}_{3}$ unless otherwise specified. ${ }^{1} \mathrm{H}$ chemical shifts are reported in ppm on the $\delta$-scale relative to $\mathrm{CDCl}_{3}(\delta 7.26)$ or $\mathrm{CD}_{3} \mathrm{OD}(3.31)$ and ${ }^{13} \mathrm{C}$ NMR chemical shifts are reported in ppm relative to $\mathrm{CDCl}_{3}(\delta 77.16)$ and $\mathrm{CD}_{3} \mathrm{OD}(49.00)$. Multiplicities are reported as (br) broad, (s) singlet, (d) doublet, (t) triplet, (q) quartet, (quin) quintet, (sext) sextet, (hept) heptet and (m) multiplet. All $J$ values were rounded to the nearest $0.1 \mathrm{~Hz}$. ESI high resolution mass spectra were recorded on an ESITOF mass spectrometer. 


\section{Experimental Procedures}

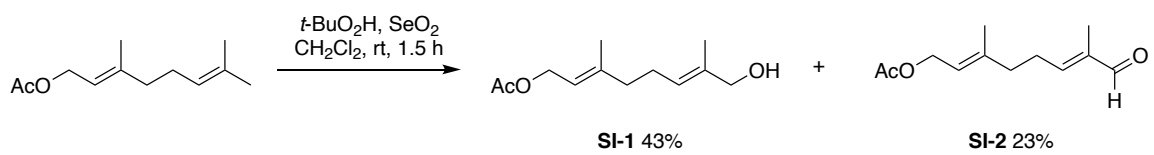

To a solution of geranyl acetate $\left(20.0 \mathrm{~g}, 102 \mathrm{mmol}, 1.0\right.$ equiv.) in $\mathrm{CH}_{2} \mathrm{Cl}_{2}(500 \mathrm{~mL})$ at room temperature was added $\mathrm{SeO}_{2}$ (4.53 g, $40.8 \mathrm{mmol}, 0.4$ equiv.), and $t-\mathrm{BuO}_{2} \mathrm{H}(55.6 \mathrm{~mL}, 306 \mathrm{mmol}, 5.50 \mathrm{M}$ in decane, 0.3 equiv.). The reaction was stirred at room temperature for $1.5 \mathrm{~h}$, then quenched with sat. $\mathrm{Na}_{2} \mathrm{~S}_{2} \mathrm{O}_{3 \text { (aq) }}$ $(500 \mathrm{~mL})$. The organic layer was separated, and further extracted with $\mathrm{CH}_{2} \mathrm{Cl}_{2}(2 \times 100 \mathrm{~mL})$. The combined organic layers were filtered, then washed with brine $(400 \mathrm{~mL})$ and the organic layer dried over $\mathrm{MgSO}_{4}$, filtered, and concentrated in vacuo. The residue was purified by flash column chromatography on $\mathrm{SiO}_{2}(5: 1 \rightarrow 1: 1$ hexanes/Et $2 \mathrm{O}$, gradient elution) to afford aldehyde $\mathbf{S I - 2}$ (4.94 g, 23\%) and the desired allylic alcohol SI-1 (9.05 g, 43\%) as yellow oils. Data for SI-1 and SI-2 matched that previously reported in the literature. ${ }^{1}$

\section{Data for SI-1:}

$\mathbf{R}_{\mathbf{f}}: 0.40$ (2:1 hexanes/EtOAc).

FTIR (neat): 3428, 2921, 1738, 1444, 1366, 1233, 1022, $954 \mathrm{~cm}^{-1}$.

${ }^{1}$ H NMR (500 MHz, CDCl $): \delta 5.36-5.33(\mathrm{~m}, 2 \mathrm{H}), 4.58(\mathrm{~d}, J=7.1 \mathrm{~Hz}, 2 \mathrm{H}), 3.99(\mathrm{~s}, 2 \mathrm{H}), 2.18-2.16$ (m, 2H), $2.10-2.08(\mathrm{~m}, 2 \mathrm{H}), 2.05$ (s 3H), 1.70 (s, 3H), 1.66 (s, 3H) ppm.

${ }^{13}$ C NMR (125 MHz, $\left.\mathbf{C D C l}_{3}\right): \delta 171.3,141.8,135.4,125.5,118.9,69.1,61.6,39.2,25.8,21.2,16.5$, $13.8 \mathrm{ppm}$.

HRMS (ESI): m/z: [M-H] Calcd for $\mathrm{C}_{12} \mathrm{H}_{19} \mathrm{O}_{3}$ 211.1340; found 211.1344.

\section{Data for S1-2:}

$\mathbf{R}_{\mathbf{f}}: 0.75(2: 1$ hexanes/EtOAc).

FTIR (neat): 2417, 2977, 1736, 1075, 1445, 1381, 1364, 1231, 1024, $845 \mathrm{~cm}^{-1}$.

${ }^{1}$ H NMR (500 MHz, CDCl $)$ ): $\delta 9.34(\mathrm{~s}, 1 \mathrm{H}), 6.40(\mathrm{t}, J=6.9 \mathrm{~Hz}, 1 \mathrm{H}), 5.34(\mathrm{t}, J=7.5 \mathrm{~Hz}, 1 \mathrm{H}), 4.54$ (d, $J=7.0 \mathrm{~Hz}, 2 \mathrm{H}), 2.45$ (q, $J=7.5 \mathrm{~Hz}, 2 \mathrm{H}), 2.19$ (t, $J=7.6 \mathrm{~Hz}, 2 \mathrm{H}), 2.00$ (s, 3H), 1.70 (s, 3H), 1.70 (s, 6H) ppm.

${ }^{13}$ C NMR (125 MHz, $\left.\mathbf{C D C l}_{3}\right): \delta$ 195.1, 171.0, 153.4, 140.4, 139.7, 119.7, 61.1, 37.8, 27.0, 21.0, 16.4, $9.2 \mathrm{ppm}$.

HRMS (ESI): m/z: $[\mathrm{M}+\mathrm{H}]^{+}$Calcd for $\mathrm{C}_{12} \mathrm{H}_{19} \mathrm{O}_{3} 211.1329$; found 211.1331 .

\footnotetext{
${ }^{1}$ Ippoliti, F. M.; Barber, J. S.; Tang, Y.; Garg, N. K. J. Org. Chem. 2018, 83, 11323.
} 


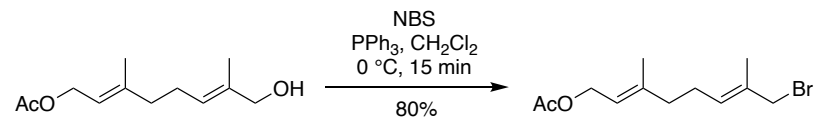

SI-1

15

To a solution of allylic alcohol SI-1 (24.0 g, $113 \mathrm{mmol}, 1.0$ equiv.) in $\mathrm{CH}_{2} \mathrm{Cl}_{2}(400 \mathrm{~mL})$ was added $\mathrm{PPh}_{3}$ (38.5 g, $147 \mathrm{mmol}, 1.3$ equiv.). The mixture was cooled to $0{ }^{\circ} \mathrm{C}$, then NBS (26.2 g, $147 \mathrm{mmol}, 1.3$ equiv.) was added portion-wise. The reaction was stirred at $0{ }^{\circ} \mathrm{C}$ for $5 \mathrm{~min}$, then warmed to room temperature and stirred for a further $10 \mathrm{~min}$. The reaction was quenched by addition of sat. $\mathrm{Na}_{2} \mathrm{~S}_{2} \mathrm{O}_{3 \text { (aq) }}$ $(300 \mathrm{~mL})$, and the organic layer separated and concentrated in vacuo. The residue was dissolved in dichloromethane $(30 \mathrm{~mL})$, then $\mathrm{Et}_{2} \mathrm{O}(200 \mathrm{~mL})$ and hexanes $(400 \mathrm{~mL})$ were added. The resultant suspension was filtered through celite, removing excess triphenylphosphine oxide, and succinamide. The filtrate was then concentrated, and residue purified by flash column chromatography on $\mathrm{SiO}_{2}(10: 1$ $\rightarrow 6: 1$ hexanes/Et $t_{2} \mathrm{O}$, gradient elution) to afford the allylic bromide $\mathbf{1 5}(25.0 \mathrm{~g}, 80 \%)$ as a clear oil. Data for $\mathbf{1 5}$ matched that previously reported in literature. ${ }^{2}$

\section{Data for 15:}

$\mathbf{R}_{\mathbf{f}}: 0.40$ (4:1 hexanes/EtOAc).

FTIR (neat): 3424, 2964, 1738, 1444, 1366, 1220, 1024, $756 \mathrm{~cm}^{-1}$.

${ }^{1} \mathrm{H}$ NMR (500 MHz, CDCl $): \delta 5.56(\mathrm{t}, J=7.0 \mathrm{~Hz}, 1 \mathrm{H}), 5.35-5.33(\mathrm{~m}, 1 \mathrm{H}), 4.58(\mathrm{dd}, J=7.1,2.1 \mathrm{~Hz}$, 2H), 3.96 (s, 2H), $2.19-2.15$ (m, 2H), $2.10-2.09$ (m, 2H), $2.06(\mathrm{~s}, 3 \mathrm{H}), 1.76(\mathrm{~s}, 3 \mathrm{H}), 1.70(\mathrm{~s}, 3 \mathrm{H}) \mathrm{ppm}$. ${ }^{13}$ C NMR (125 MHz, CDCl $\left._{3}\right): \delta 171.2,141.5,132.6,130.6,119.0,61.4,41.7,38.7,26.6,21.2,16.6$, $14.8 \mathrm{ppm}$.

\footnotetext{
${ }^{2}$ Oehlschlager, A. C.; Wong, J. W.; Verigin, V. G.; Pierce, H. D., Jr. J. Org. Chem. 1983, 48, 5009.
} 


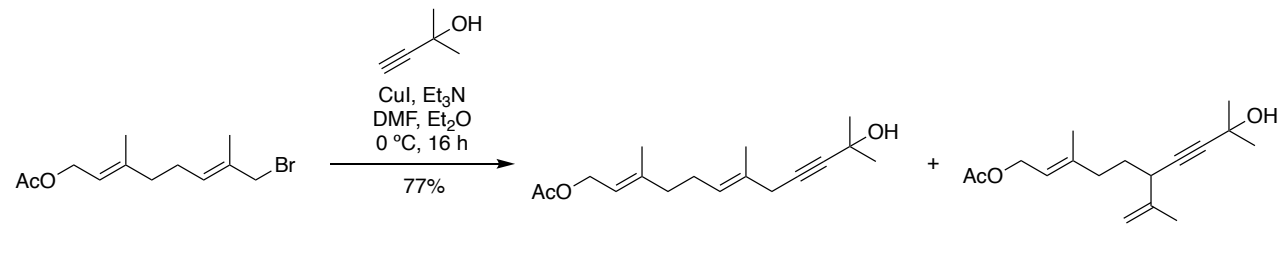

15

16

SI-3

Using modified conditions from Hodgson et al., ${ }^{3} \mathrm{CuI}$ (13.0 g, $68.2 \mathrm{mmol}, 1.2$ equiv.) was added to a solution of 2-methylbut-3-yn-2-ol (6.60 mL, $68.2 \mathrm{mmol}, 1.2$ equiv.) and $\mathrm{Et}_{3} \mathrm{~N}$ (9.50 mL, $68.2 \mathrm{mmol}$, 1.2 equiv.) in $\mathrm{DMF}(80 \mathrm{~mL})$ and $\mathrm{Et}_{2} \mathrm{O}(80 \mathrm{~mL})$ and the reaction mixture was stirred for $1 \mathrm{~h}$ at room temperature. A solution of allylic bromide 15 (14.8 g, $56.7 \mathrm{mmol}, 1.0$ equiv.) in $\mathrm{Et}_{2} \mathrm{O}(80 \mathrm{~mL})$ was added to the reaction mixture and stirred for a further $16 \mathrm{~h}$ at room temperature. The reaction mixture was quenched with sat. $\mathrm{NH}_{4} \mathrm{Cl}_{(\mathrm{aq})}(80 \mathrm{~mL})$. The aqueous layer was extracted with $\mathrm{Et}_{2} \mathrm{O}(3 \times 80 \mathrm{~mL})$ and the combined organic extracts were dried with $\mathrm{MgSO}_{4}$, concentrated under reduced pressure and purified by flash column chromatography on $\mathrm{SiO}_{2}(4: 1$ hexanes/EtOAc) to give a yellow oil of the propargylic alcohols $\mathbf{1 6}$ and SI-3 (9.97 g, 77\%) as a 1.2:1 mixture. These products were then taken on as a crude mixture and used without further purification.

\section{Data for 16:}

$\mathbf{R}_{\mathbf{f}}: 0.20$ (20:1, hexanes:EtOAc).

FTIR (neat): 3428, 2981, 1736, 1443, 1365, 1232, 1167, 1023, 949, 754, $667 \mathrm{~cm}^{-1}$.

${ }^{1}$ H NMR (500 MHz, CDCl$\left.)_{3}\right): \delta 5.35-5.34(\mathrm{~m}, 2 \mathrm{H}), 4.59(\mathrm{~d}, J=7.0 \mathrm{~Hz}, 2 \mathrm{H}), 2.87(\mathrm{~s}, 1 \mathrm{H}), 2.14(\mathrm{t}, J=$ $7.2 \mathrm{~Hz}, 2 \mathrm{H}), 2.07-2.05(\mathrm{~m}, 2 \mathrm{H}), 2.05$ (s, 3H), 1.71 (s 3H), 1.65 (s, 3H), 1.51 (s, 6H) ppm.

${ }^{13}$ C NMR (125 MHz, $\left.\mathbf{C D C l}_{3}\right): \delta 171.3,141.7,130.5,125.1,118.7,112.2,87.6,80.0,65.4,61.5,39.3$, $31.9,28.7,26.2,21.1,19.8,16.2 \mathrm{ppm}$.

HRMS (ESI): $\mathrm{m} / \mathrm{z}$ : [M+Na] $]^{+}$Calcd for $\mathrm{C}_{17} \mathrm{H}_{26} \mathrm{O}_{3} \mathrm{Na}$ 301.1774; found 301.1777.

\footnotetext{
${ }^{3}$ Hodgson, D. M.; Talbot, E. P. A.; Clark, B. P. Org. Lett. 2011, 13, 5751.
} 


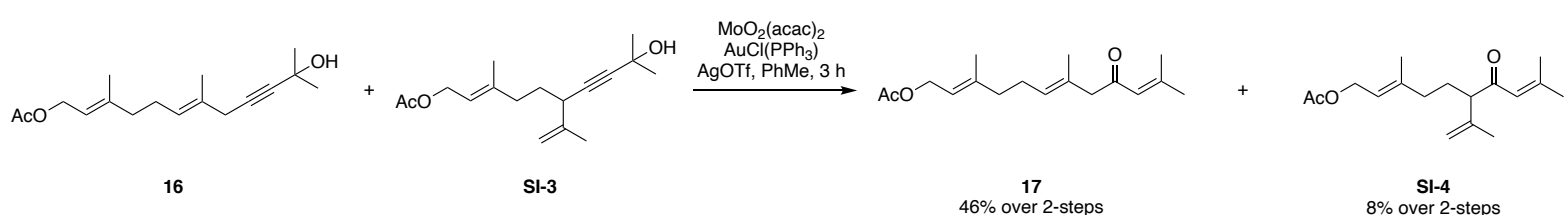

Using modified conditions from Hodgson et al., ${ }^{3} \mathrm{MoO}_{2}(\mathrm{acac})_{2}$ (712 mg, $1.66 \mathrm{mmol}, 0.05$ equiv.), $\mathrm{AuCl}\left(\mathrm{PPh}_{3}\right)(1.07 \mathrm{mg}, 2.18 \mathrm{mmol}, 0.05$ equiv.) and AgOTf (560 mg, $2.18 \mathrm{mmol}, 0.05$ equiv.) were added successively to a 1.2:1 mixture of alkyne $\mathbf{1 6}$ and SI-3 (9.97 g, $43.7 \mathrm{mmol}, 1.0$ equiv.) in dry toluene $(350 \mathrm{~mL})$ at $0{ }^{\circ} \mathrm{C}$. The reaction mixture was stirred at room temperature for $3 \mathrm{~h}$ then filtered through a pad of Celite, washed through with $\mathrm{Et}_{2} \mathrm{O}(500 \mathrm{~mL})$. The filtrate was concentrated under reduced pressure and purified by flash column chromatography on $\mathrm{SiO}_{2}(9: 1$ hexanes/EtOAc) to afford SI-4 (904 mg, 8\% over 2 steps) and $\mathbf{1 7}$ (5.94 g, 46\% over 2 steps) as a yellow oil.

\section{Data for 17:}

$\mathbf{R}_{\mathbf{f}}: 0.20$ (9:1 hexanes/EtOAc).

FTIR (neat): 2975, 1738, 1685, 1618, 1446, 1380, 1333, 1233, 1024, 915, $732 \mathrm{~cm}^{-1}$.

${ }^{1}$ H NMR (500 MHz, CDCl $): \delta 6.11-6.10(\mathrm{~m}, 1 \mathrm{H}), 5.36-5.33(\mathrm{~m}, 1 \mathrm{H}), 5.26-5.22(\mathrm{~m}, 1 \mathrm{H}), 4.58$ (d, $J=6.9 \mathrm{~Hz}, 2 \mathrm{H}), 3.04(\mathrm{~s}, 2 \mathrm{H}), 2.21-2.15(\mathrm{~m}, 2 \mathrm{H}), 2.14$ (d, $J=1.3 \mathrm{~Hz}, 2 \mathrm{H}), 2.10-2.07$ (m, 2H), 2.05 (s, 3H), $1.88(\mathrm{~d}, J=1.3 \mathrm{~Hz}, 3 \mathrm{H}), 1.71$ (s, 3H), 1.62 (s, 3H) ppm.

${ }^{13}$ C NMR (125 MHz, CDCl $): \delta$ 199.4, 171.3, 155.8, 142.1, 130.3, 128.6, 123.0, 118.7, 61.5, 55.5, $39.3,27.8,26.5,21.2,20.8,16.6,16.6 \mathrm{ppm}$.

HRMS (ESI): $\mathrm{m} / \mathrm{z}$ : [M+Na $]^{+}$Calcd for $\mathrm{C}_{17} \mathrm{H}_{26} \mathrm{O}_{3} \mathrm{Na} 301.1774$; found 301.1777.

\section{Data for SI-4:}

$\mathbf{R}_{\mathbf{f}}: 0.30$ (9:1 hexanes/EtOAc).

FTIR (neat): 2936, 1737, 1685, 1621, 1440, 1367, 1230, 1022, 967, $912 \mathrm{~cm}^{-1}$.

${ }^{1}$ H NMR (500 MHz, CDCl $): \delta 6.11-6.10(\mathrm{~m}, 1 \mathrm{H}), 5.30(\mathrm{t}, J=7.3 \mathrm{~Hz}, 1 \mathrm{H}), 4.90(\mathrm{t}, J=1.6 \mathrm{~Hz}, 1 \mathrm{H})$, $4.86-4.87$ (m, 1H), 4.54 (d, $J=7.0 \mathrm{~Hz}, 2 \mathrm{H}), 3.03-3.00$ (m, 1H), 2.10 (s, 3H), 2.01 (s, 3H), $1.92-$ $1.90(\mathrm{~m}, 3 \mathrm{H}), 1.84$ (s, 3H), 1.65 (s, 3H), 1.60 (s, 3H), $1.61-1.55$ (m, 1H) ppm.

${ }^{13}$ C NMR (125 MHz, $\mathbf{C D C l}$ ): $\delta$ 200.1, 171.1, 155.9, 143.2, 141.9, 122.9, 118.8, 114.7, 61.4, 60.9, $37.2,27.8,26.5,21.1,20.8,19.9,16.4 \mathrm{ppm}$.

HRMS (ESI): $\mathrm{m} / \mathrm{z}$ : $[\mathrm{M}+\mathrm{H}]^{+}$Calcd for $\mathrm{C}_{17} \mathrm{H}_{27} \mathrm{O}_{3} 279.1955$; found 279.1956 . 


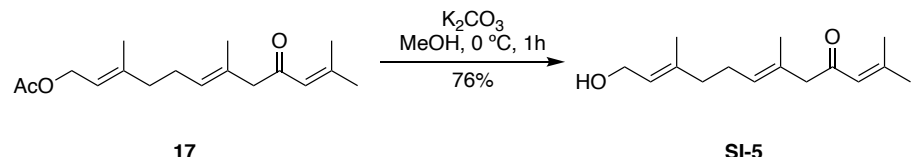

To a solution of ketone $17\left(1.01 \mathrm{~g}, 3.63 \mathrm{mmol}, 1.0\right.$ equiv.) in $\mathrm{MeOH}(20 \mathrm{~mL})$ at $0{ }^{\circ} \mathrm{C}$ was added $\mathrm{K}_{2} \mathrm{CO}_{3}$ (501 mg, $3.63 \mathrm{mmol}, 1.0$ equiv.) portion-wise. The reaction was stirred at $0{ }^{\circ} \mathrm{C}$ for $1 \mathrm{~h}$, brine $(50 \mathrm{~mL})$ was added and the mixture was extracted with $\mathrm{Et}_{2} \mathrm{O}(30 \mathrm{~mL} \times 3)$. The combined organic extracts were washed with brine $(30 \mathrm{~mL} \times 3)$, dried over $\mathrm{MgSO}_{4}$, filtered, and concentrated in vacuo. The residue was purified by flash column chromatography on $\mathrm{SiO}_{2}(4: 1 \rightarrow 2: 1$ hexanes/EtOAc) to afford alcohol SI-5 as a clear colourless oil (654 mg, 76\%).

\section{Data for SI-5:}

$\mathbf{R}_{\mathbf{f}}: 0.20$ (4:1 hexanes/EtOAc).

FTIR (neat): $3500,2915,1685,1618,1445,1007 \mathrm{~cm}^{-1}$.

${ }^{1}$ H NMR (500 MHz, CDCl $): \delta 6.08-6.07(\mathrm{~m}, 1 \mathrm{H}), 5.40-5.37(\mathrm{~m}, 1 \mathrm{H}), 5.20-5.17(\mathrm{~m}, 1 \mathrm{H}), 4.12$ (d, $J=6.9 \mathrm{~Hz}, 2 \mathrm{H}), 3.02(\mathrm{~s}, 2 \mathrm{H}), 2.18-2.14(\mathrm{~m}, 2 \mathrm{H}), 2.11(\mathrm{~s}, 3 \mathrm{H}), 2.05(\mathrm{t}, J=7.6 \mathrm{~Hz}, 2 \mathrm{H}), 1.86(\mathrm{~s}$, $3 \mathrm{H}), 1.65$ (s, 3H), $1.59(\mathrm{~s}, 3 \mathrm{H}) \mathrm{ppm}$.

${ }^{13}$ C NMR (125 MHz, CDCl $): \delta 199.5,155.9,138.9,130.0,128.8,124.1,123.1,59.4,55.3,39.2,27.8$, 26.4, 20.8, 16.6, $16.3 \mathrm{ppm}$.

HRMS (ESI): $\mathrm{m} / \mathrm{z}:$ [M+Na] ${ }^{+}$Calcd for $\mathrm{C}_{15} \mathrm{H}_{24} \mathrm{O}_{2} \mathrm{Na} 259.1669$; found 259.1670. 


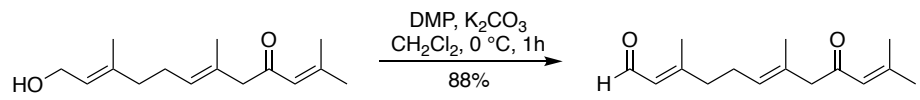

SI-5

14

To a solution of alcohol SI-5 (654 mg, $2.77 \mathrm{mmol}, 1.0$ equiv.) in $\mathrm{CH}_{2} \mathrm{Cl}_{2}(20 \mathrm{~mL})$ at $0{ }^{\circ} \mathrm{C}$ was added $\mathrm{NaHCO}_{3}$ (465 mg, $5.53 \mathrm{mmol}, 2.0$ equiv.), followed by Dess-Martin periodinane (1.76 g, $4.15 \mathrm{mmol}$, 1.5 equiv.). The reaction was stirred at $0{ }^{\circ} \mathrm{C}$ for $1 \mathrm{~h}$, then hexanes $(80 \mathrm{~mL})$ was added. The resultant suspension was filtered, and the filtrate was concentrated in vacuo. The residue was purified by flash column chromatography on $\mathrm{SiO}_{2}(4: 1$ hexanes/EtOAc) to afford aldehyde $\mathbf{1 4}$ as a pale, light yellow oil (573 mg, 88\%).

\section{Data for 14:}

Rf: 0.40 ( $7: 3$ hexanes/EtOAc).

FTIR (neat): 2915, 1673, 1619, 1445, 1383, 1194, $1121 \mathrm{~cm}^{-1}$.

${ }^{1}$ H NMR (500 MHz, CDCl $\left.\mathbf{~}_{3}\right): \delta 9.99(\mathrm{~d}, J=8.0 \mathrm{~Hz}, 1 \mathrm{H}), 6.08(\mathrm{~s}, 1 \mathrm{H}), 5.88(\mathrm{~d}, J=8.0 \mathrm{~Hz}, 1 \mathrm{H}), 5.22$ $5.20(\mathrm{~m}, 1 \mathrm{H}), 3.04(\mathrm{~s}, 2 \mathrm{H}), 2.29-2.26(\mathrm{~m}, 4 \mathrm{H}), 2.17(\mathrm{~s}, 3 \mathrm{H}), 2.14(\mathrm{~s}, 3 \mathrm{H}), 1.88(\mathrm{~s}, 3 \mathrm{H}), 1.63(\mathrm{~s}, 3 \mathrm{H})$ ppm.

${ }^{13}$ C NMR (125 MHz, CDCl $): \delta 199.1,191.4,163.5,156.2,131.4,127.7,127.3,123.0,55.2,40.4$, 27.9, 25.9, 20.9, 17.7, $16.7 \mathrm{ppm}$.

HRMS (ESI): $\mathrm{m} / \mathrm{z}:[\mathrm{M}+\mathrm{Na}]^{+}$Calcd for $\mathrm{C}_{15} \mathrm{H}_{22} \mathrm{O}_{2} \mathrm{Na}$ 257.1512; found 257.1515. 


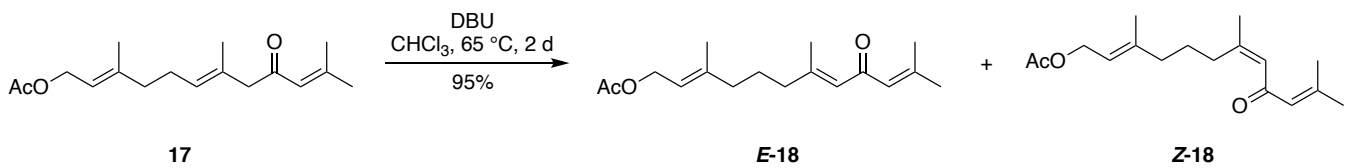

To a solution of 17 (1.21 g, $4.35 \mathrm{mmol}, 1.0$ equiv.) in $\mathrm{CHCl}_{3}(20 \mathrm{~mL})$ was added $\mathrm{DBU}(0.80 \mathrm{~mL}, 5.21$ mmol, 1.2 equiv.) and the reaction was left to stir at reflux for $2 \mathrm{~d}$ using an aluminium heating block. The resulting solution was then concentrated and purified by flash column chromatography on $\mathrm{SiO}_{2}$ (4:1 hexanes/Et $\mathrm{t}_{2} \mathrm{O}$ ) to afford a yellow oil of $\boldsymbol{E}-\mathbf{1 8}$ and $\boldsymbol{Z}-\mathbf{1 8}$ as a 2.2:1 mixture of diastereomers (1.15 g, $95 \%$ ). Although these products were then taken on as a crude mixture and used without further purification, it was found that a small sample of pure material could be isolated for analytical characterisation purposes.

\section{Data for $E$-18:}

$\mathbf{R}_{\mathbf{f}}: 0.25$ (4:1 hexanes/EtOAc).

FTIR (neat): 2936, 1737, 1672, 1443, 1380, 1229, 1108, 1022, 955, 877, $756 \mathrm{~cm}^{-1}$.

${ }^{1}$ H NMR (500 MHz, CDCl $)$ ): $\delta 6.05(\mathrm{~m}, 1 \mathrm{H}), 6.01(\mathrm{~m}, 1 \mathrm{H}), 5.36-5.30(\mathrm{~m}, 1 \mathrm{H}), 4.57(\mathrm{dd}, J=7.2,2.4$ $\mathrm{Hz}, 2 \mathrm{H}), 2.15$ (s, 3H), 2.14 (s, 3H), $2.10-2.06$ (m, 2H), 2.04 (s, 3H), $2.04-2.00$ (m, 2H), 1.88 (s, 3H), $1.63-1.58(\mathrm{~m}, 2 \mathrm{H}) \mathrm{ppm}$.

${ }^{13}$ C NMR (125 MHz, CDCl $): \delta$ 191.7, 171.2, 157.5, 154.5, 141.8, 126.4, 125.9, 119.0, 61.4, 40.8, $39.1,27.9,25.5,21.1,20.7,19.2,16.4 \mathrm{ppm}$.

HRMS (ESI): m/z: [M+Na] ${ }^{+}$Calcd for $\mathrm{C}_{17} \mathrm{H}_{26} \mathrm{O}_{3} \mathrm{Na}$ 301.1774; found 301.1773 .

\section{Data for $Z$-18:}

$\mathbf{R}_{\mathbf{f}}: 0.30$ (4:1 hexanes/EtOAc).

FTIR (neat): 2936, 1736, 1672, 1623, 1444, 1378, 1228, 1112, 1023, 1023, 955, 870, $772 \mathrm{~cm}^{-1}$.

${ }^{1}$ H NMR (500 MHz, CDCl $\left.\mathbf{H}_{3}\right): \delta 6.02(\mathrm{~m}, 2 \mathrm{H}), 5.36-5.30(\mathrm{~m}, 1 \mathrm{H}), 4.56(\mathrm{~d}, J=6.8 \mathrm{~Hz}, 2 \mathrm{H}), 2.61-$ $2.54(\mathrm{~m}, 2 \mathrm{H}), 2.14(\mathrm{~s}, 3 \mathrm{H}), 2.11-2.05(\mathrm{~m}, 2 \mathrm{H}), 2.03(\mathrm{~s}, 3 \mathrm{H}), 1.86(\mathrm{dd}, J=5.6,1.3 \mathrm{~Hz}, 6 \mathrm{H}), 1.69$ (s, $3 \mathrm{H}), 1.59(\mathrm{~m}, 2 \mathrm{H}) \mathrm{ppm}$.

${ }^{13}$ C NMR (125 MHz, $\mathbf{C D C l}$ ): $\delta$ 191.7, 171.2, 157.5, 154.5, 142.0, 126.6, 126.4, 118.7, 61.5, 39.3, $34.4,27.8,26.5,25.5,21.1,20.7,16.5 \mathrm{ppm}$.

HRMS (ESI): m/z: [M+Na] ${ }^{+}$Calcd for $\mathrm{C}_{17} \mathrm{H}_{26} \mathrm{O}_{3} \mathrm{Na}$ 301.1774; found 301.1774. 


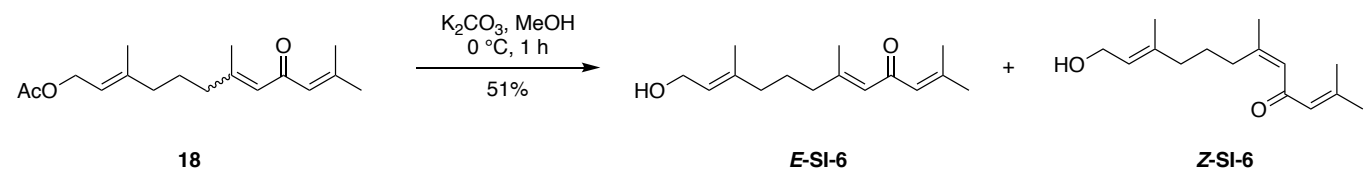

To a solution of $\boldsymbol{E}-\mathbf{1 8}$ and $\boldsymbol{Z}-\mathbf{1 8}$ as a 2.2:1 mixture of diastereomers (1.15 g, $4.13 \mathrm{mmol}, 1.0$ equiv.) in $\mathrm{MeOH}(20 \mathrm{~mL})$ was added portion-wise $\mathrm{K}_{2} \mathrm{CO}_{3}(0.57 \mathrm{~g}$, $4.13 \mathrm{mmol}, 1.0$ equiv. $)$ at $0{ }^{\circ} \mathrm{C}$. The reaction was left to stir for $1 \mathrm{~h}$, then quenched upon addition of distilled water $(30 \mathrm{~mL})$. Extraction with $\mathrm{CHCl}_{3}$ ( $3 \times 30 \mathrm{~mL}$ ) was performed, and the solution was dried with $\mathrm{MgSO}_{4}$, concentrated and purified by flash column chromatography on $\mathrm{SiO}_{2}(3: 1$ hexanes/EtOAc) to afford a yellow oil of $\boldsymbol{E}$-SI-6 and $\boldsymbol{Z}$-SI-6 as a 2.2:1 mixture of diastereoisomers $(502 \mathrm{mg}, 51 \%)$. Although these products were then taken on as a crude mixture and used without further purification, it was found that a small sample of pure material could be isolated for analytical characterisation purposes.

\section{Data for $E$-SI-6:}

$\mathbf{R}_{\mathbf{f}}: 0.20$ (3:1 hexane/EtOAc).

FTIR (neat): 3410, 2935, 1671, 1443, 1381, 1218, 1112, 1005, $875 \mathrm{~cm}^{-1}$.

${ }^{1}$ H NMR (500 MHz, CDCl$): \delta 6.04-6.04(\mathrm{~m}, 2 \mathrm{H}), 5.41-5.38(\mathrm{~m}, 1 \mathrm{H}), 4.14(\mathrm{~d}, J=6.7 \mathrm{~Hz}, 1 \mathrm{H})$, 2.175 (s, 6H), 2.11 (t, $J=7.7 \mathrm{~Hz}, 2 \mathrm{H}), 2.02$ (t, $J=7.6 \mathrm{~Hz}, 2 \mathrm{H}), 1.89$ (s, 3H), 1.67 (s, 3H), $1.63-1.58$ $(\mathrm{m}, 2 \mathrm{H}) \mathrm{ppm}$.

${ }^{13}$ C NMR (125 MHz, CDCl $)$ : $\delta 191.8,157.7,154.5,139.1,126.4,125.9,124.1,59.4,40.9,39.1,27.9$, 26., 25.6, 19.2, $16.3 \mathrm{ppm}$.

HRMS (ESI): $\mathrm{m} / \mathrm{z}:[\mathrm{M}+\mathrm{Na}]^{+}$Calcd for $\mathrm{C}_{15} \mathrm{H}_{24} \mathrm{O}_{2} \mathrm{Na} 259.1669$; found 259.1668.

\section{Data for Z-SI-6:}

$\mathbf{R}_{\mathbf{f}}: 0.20$ (3:1 hexanes/EtOAc).

FTIR (neat): 3411, 2933, 1670, 1620, 1443, 1379, 1221, 1112, 1000, 870, $772 \mathrm{~cm}^{-1}$.

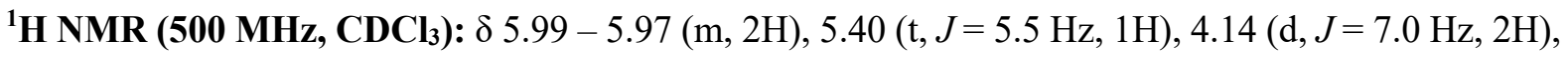
$2.61-2.57$ (m, 2H), $2.21-2.14(\mathrm{br} \mathrm{s}, 1 \mathrm{H}), 2.10(\mathrm{~s}, 3 \mathrm{H}), 2.02(\mathrm{t}, J=7.5 \mathrm{~Hz}, 2 \mathrm{H}), 1.84-1.81(\mathrm{~m}, 6 \mathrm{H})$, $1.63(\mathrm{~s}, 3 \mathrm{H}), 1.58-1.51(\mathrm{~m}, 2 \mathrm{H}) \mathrm{ppm}$.

${ }^{13}$ C NMR (125 MHz, $\left.\mathbf{C D C l}_{3}\right): \delta 191.8,157.7,154.5,139.1,126.4,123.9,59.4,59.4,39.2,32.8,27.8$, 26.2, 25.5, 20.7, $16.3 \mathrm{ppm}$.

HRMS (ESI): m/z: [M+H] $]^{+}$Calcd for $\mathrm{C}_{15} \mathrm{H}_{24} \mathrm{O}_{2} 235.1693$; found 235.1697. 


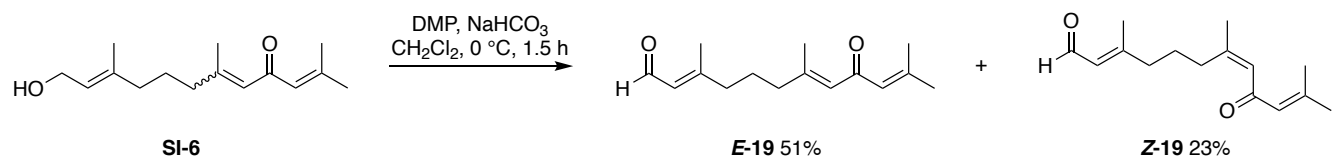

To a solution of $\boldsymbol{E}$-SI-6 and $\boldsymbol{Z}$-SI-6 as a 2.2:1 mixture of diastereomers (502 mg, $2.12 \mathrm{mmol}, 1.0$ equiv.) in $\mathrm{CH}_{2} \mathrm{Cl}_{2}(30 \mathrm{~mL})$ at $0{ }^{\circ} \mathrm{C}$ was added $\mathrm{NaHCO}_{3}(360 \mathrm{mg}, 4.25 \mathrm{mmol}, 2.0$ equiv.) and Dess-Martin periodinane ( $1.35 \mathrm{~g}, 3.18 \mathrm{mmol}, 1.5$ equiv.). The solution was left to stir for $1.5 \mathrm{~h}$ then quenched upon addition of sat. $\mathrm{NaHCO}_{3(\mathrm{aq})}(30 \mathrm{~mL})$. The organic layer was extracted with $\mathrm{CH}_{2} \mathrm{Cl}_{2}(3 \times 30 \mathrm{~mL})$, dried with $\mathrm{MgSO}_{4}$ and concentrated in vacuo. Purification by flash column chromatography on $\mathrm{SiO}_{2}(4: 1$ hexanes/ $\mathrm{Et}_{2} \mathrm{O}$ ) then afforded $\boldsymbol{Z}-\mathbf{1 9}$ (113 mg, 12\% over 2 steps) and $\boldsymbol{E}-\mathbf{1 9}$ (252 mg, 26\% over 2 steps) as yellow oils. If needed further recrystallisation of the trans isomer $\boldsymbol{E}-\mathbf{1 9}$ with hexanes at $0{ }^{\circ} \mathrm{C}$ could also be performed (oil at room temperature).

\section{Data for $E-19$ :}

$\mathbf{R}_{\mathbf{f}}: 0.15$ (4:1 hexanes/Et $\left.{ }_{2} \mathrm{O}\right)$.

FTIR (neat): 2941, 1671, 1625, 1443, 1382, 1217, 1195, 1109, 1044, $873 \mathrm{~cm}^{-1}$.

${ }^{1}$ H NMR (500 MHz, CDCl $)$ : $\delta 10.00(\mathrm{~d}, J=7.9 \mathrm{~Hz}, 1 \mathrm{H}), 6.07-6.06(\mathrm{~m}, 1 \mathrm{H}), 6.03-6.02(\mathrm{~m}, 1 \mathrm{H})$, $5.89(\mathrm{dq}, J=7.9,1.2 \mathrm{~Hz}, 1 \mathrm{H}), 2.23-2.19(\mathrm{~m}, 2 \mathrm{H}), 2.19-2.13(\mathrm{~m}, 8 \mathrm{H}), 1.90(\mathrm{~s}, 3 \mathrm{H}), 1.73-1.70$ (m, 3H) $\mathrm{ppm}$.

${ }^{13}$ C NMR (125 MHz, $\mathbf{C D C l}$ ): $\delta$ 191.6, 191.3, 163.2, 156.4, 155.0, 127.7, 126.3, 126.3, 40.7, 40.0, 27.9, 25.0, 20.7, 19.1, $17.7 \mathrm{ppm}$.

HRMS (ESI): m/z: [M+H] $]^{+}$Calcd for $\mathrm{C}_{15} \mathrm{H}_{23} \mathrm{O}_{2}$ 235.1693; found 235.1696.

\section{Data for $Z$-19:}

$\mathbf{R}_{\mathbf{f}}: 0.20$ (4:1 hexanes/ $\left.\mathrm{Et}_{2} \mathrm{O}\right)$.

FTIR (neat): 2935, 1670, 1444, 1380, 1194, 1113, 1045, 872, $773 \mathrm{~cm}^{-1}$.

${ }^{1} \mathbf{H}$ NMR (500 MHz, $\left.\mathbf{C D C l}_{3}\right): \delta 9.99(\mathrm{~d}, J=8.0 \mathrm{~Hz}, 1 \mathrm{H}), 6.06-6.04(\mathrm{~m}, 2 \mathrm{H}), 5.89(\mathrm{~d}, J=8.1 \mathrm{~Hz}, 1 \mathrm{H})$, $2.62(\mathrm{t}, J=7.8,2 \mathrm{H}), 2.28(\mathrm{t}, J=7.8 \mathrm{~Hz}, 2 \mathrm{H}), 2.19$ (s, 3H), 2.15 (s, 3H), 1.88 (s, 6H), 1.69 (pent, $J=7.8$ $\mathrm{Hz}, 2 \mathrm{H}) \mathrm{ppm}$.

${ }^{13}$ C NMR (125 MHz, $\left.\mathbf{C D C l}_{3}\right): \delta$ 191.5, 191.1, 164.2, 157.2, 154.8, 127.5, 126.9, 126.3, 40.7, 33.2, 27.9, 25.8, 25.5, 20.7, $17.7 \mathrm{ppm}$.

HRMS (ESI): m/z: [M+H] $]^{+}$Calcd for $\mathrm{C}_{15} \mathrm{H}_{23} \mathrm{O}_{2}$ 235.1693; found 235.1697. 


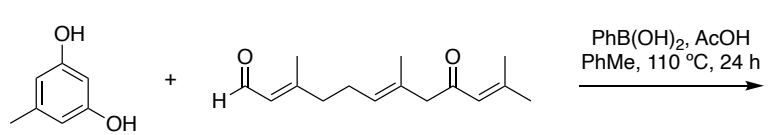

13: orcinol

14

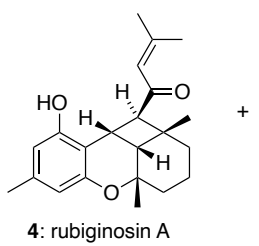

$10 \%$

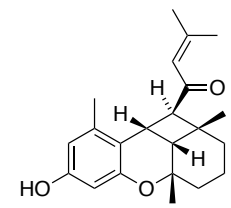

26: "iso-rubiginosin $\mathrm{A}$ " $31 \%$

To a solution of aldehyde 14 (146 mg, $0.623 \mathrm{mmol}, 1.0$ equiv.) in PhMe (10 mL) was added orcinol monohydrate 13 (116 mg, $0.935 \mathrm{mmol}, 1.5$ equiv.), phenylboronic acid (114 mg, $0.935 \mathrm{mmol}, 1.5$ equiv.) and acetic acid ( $0.04 \mathrm{~mL}, 0.623 \mathrm{mmol}, 1.0$ equiv.). The solution was heated to reflux using an aluminium heating block and left to stir at $110^{\circ} \mathrm{C}$ for $24 \mathrm{~h}$, then concentrated in vacuo. The residue was purified by flash column chromatography on $\mathrm{SiO}_{2}(20: 1 \rightarrow$ 9:1 hexanes/EtOAc, gradient elution) to afford rubiginosin A (4) (22 mg, 10\%) and "iso-rubiginosin A" (26) (65 mg, 31\%) as white solids. Data for rubiginosin A (4) matched that previously reported in the literature. ${ }^{4}$

\section{Data for rubiginosin A (4):}

MP: $137.5-138.9^{\circ} \mathrm{C}\left(\right.$ lit. $\left.142-144^{\circ} \mathrm{C}\right) .^{4}$

$\mathbf{R}_{\mathbf{f}}: 0.40$ (9:1 hexanes/EtOAc).

FTIR (neat): 2248, 2929, 1728, 1660, 1608, 1584, 1447, 1368, 1323, 1187, 1132, 1062, $987 \mathrm{~cm}^{-1}$.

${ }^{1} \mathbf{H}$ NMR (600 MHz, CDCl$)$ ): $\delta 7.48(\mathrm{br} \mathrm{s}, 1 \mathrm{H}), 6.40(\mathrm{~s}, 1 \mathrm{H}), 6.30(\mathrm{~s}, 1 \mathrm{H}), 5.87(\mathrm{~m}, 1 \mathrm{H}), 3.82(\mathrm{t}, J=$ $9.3 \mathrm{~Hz}, 1 \mathrm{H}), 2.96(\mathrm{~d}, J=9.3 \mathrm{~Hz}, 1 \mathrm{H}), 2.23$ (s, 3H), 2.16 (s, 3H), $2.01-1.93$ (m, 1H), 2.09 (dt, $J=13.9$, $3.7 \mathrm{~Hz}) 1.90$ (s, 3H), $1.89-1.84(\mathrm{~m}, 1 \mathrm{H}), 1.82(\mathrm{~d}, J=9.8 \mathrm{~Hz}, 1 \mathrm{H}), 1.70-1.62(\mathrm{~m}, 1 \mathrm{H}), 1.37-1.24$ (m, $2 \mathrm{H}), 1.15$ (s, 3H), 1.14 (s, 3H) ppm.

${ }^{13}$ C NMR (150 MHz, $\left.\mathbf{C D C l}_{3}\right): \delta 203.2,158.1,154.4,153.4,138.0,122.4,112.1,111.5,110.6,75.3$, 58.0, 45.1, 39.5, 36.4, 34.4, 29.7, 28.2, 25.9, 24.0, 21.6, 21.5, 17.4 ppm.

HRMS (ESI): m/z: [M+H] $]^{+}$Calcd for $\mathrm{C}_{22} \mathrm{H}_{29} \mathrm{O}_{3} 341.2111$; found 341.2113.

\section{Data for "iso-rubiginosin A" (26):}

$\mathbf{R}_{\mathbf{f}}: 0.30$ ( $3: 1$ hexanes/EtOAc).

MP: $184.2-186.4^{\circ} \mathrm{C}$.

FTIR (neat): 3407, 2930, 1667, 1615, 1445, 1379, 1328, 1130, $985 \mathrm{~cm}^{-1}$.

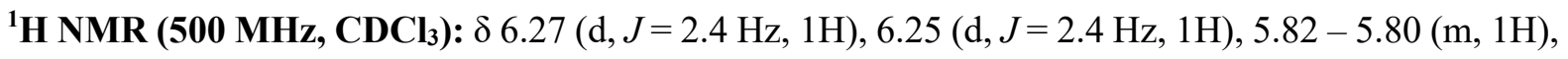
4.65 (br s, 1H), 4.02 (t, $J=8.9 \mathrm{~Hz}, 1 \mathrm{H}), 3.19$ (d, $J=9.1 \mathrm{~Hz}, 1 \mathrm{H}), 2.38$ (s, 3H), 2.11 (s, 3H), $2.09-2.02$ (m, 2H), $1.84(\mathrm{~s}, 3 \mathrm{H}), 1.84-1.81(\mathrm{~m}, 1 \mathrm{H}), 1.78(\mathrm{~d}, J=8.9 \mathrm{~Hz}, 1 \mathrm{H}), 1.67-1.61(\mathrm{~m}, 1 \mathrm{H}), 1.37-1.31$ (m, 1H), $1.25-1.17(\mathrm{~m}, 1 \mathrm{H}), 1.08(\mathrm{~s}, 3 \mathrm{H}), 0.99$ (s, 3H) ppm.

${ }^{13}$ C NMR (120 MHz, $\left.\mathbf{C D C l}_{3}\right): \delta 200.0,156.1,154.5,154.4,137.2,124.2,120.4,110.4,104.0,75.8$, 57.7, 47.7, 38.9, 37.0, 34.4, 28.7, 27.8, 26.6, 25.6, 20.9, 19.3, 17.3 ppm.

HRMS (ESI): m/z: [M+H] $]^{+}$Calcd for $\mathrm{C}_{22} \mathrm{H}_{29} \mathrm{O}_{3} 341.2111$; found 341.2114.

\footnotetext{
${ }^{4}$ Yang, Y.-X.; Wang, J.-X.; Wang, Q.; Li, H.-L.; Tao, M.; Luo, Q.; Liu, H. Fitoterapia 2018, 127, 396.
} 


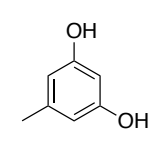

13: orcinol

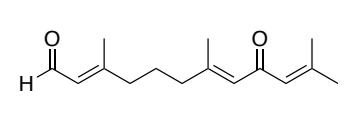

$E-19$
$\mathrm{PhB}(\mathrm{OH})_{2}, \mathrm{AcOH}$
$\mathrm{PhMe}, 110^{\circ} \mathrm{C}, 24 \mathrm{~h}$ $\stackrel{\mathrm{PhMe}, 110^{\circ} \mathrm{C}, 24 \mathrm{~h}}{\longrightarrow}$

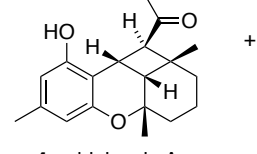

4: rubiginosin A

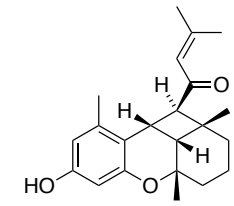

26: "iso-rubiginosin A"

To a solution of aldehyde $\boldsymbol{E} \mathbf{- 1 9}$ ( $82 \mathrm{mg}, 0.350 \mathrm{mmol}, 1.0$ equiv.) in PhMe (4 mL) was added orcinol monohydrate 13 ( $65 \mathrm{mg}, 0.525 \mathrm{mmol}, 1.5$ equiv.), phenylboronic acid (64 mg, $0.525 \mathrm{mmol}, 1.5$ equiv.) and acetic acid $(0.02 \mathrm{~mL}, 0.350 \mathrm{mmol}, 1.0$ equiv.). The solution was heated to reflux using an aluminium heating block and left to stir at $110^{\circ} \mathrm{C}$ for $24 \mathrm{~h}$, then concentrated in vacuo. The residue was purified by flash column chromatography on $\mathrm{SiO}_{2}(20: 1 \rightarrow 9: 1$ hexanes/EtOAc, gradient elution) to afford rubiginosin A (4) (16 mg, 13\%) and "iso-rubiginosin A" (26) (41 mg, 34\%) as white solids. Data for rubiginosin A (4) matched that previously reported in the literature. ${ }^{4}$ Data for 26 matched that previously obtained. 


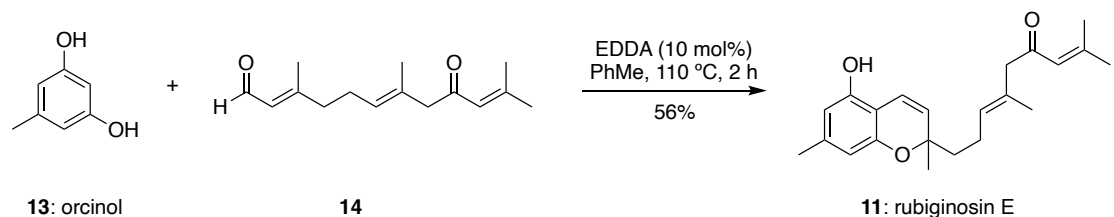

To a solution of aldehyde 14 (287 mg, $1.22 \mathrm{mmol}, 1.0$ equiv.) in PhMe (10 mL) was added orcinol monohydrate 13 (228 mg, $1.84 \mathrm{mmol}, 1.5$ equiv.), and EDDA (22 mg, $0.122 \mathrm{mmol}, 0.10$ equiv.). The mixture was heated to reflux using an aluminium heating block for $2 \mathrm{~h}$, and the solvent was removed in vacuo. The residue was purified by flash column chromatography on $\mathrm{SiO}_{2}(4: 1 \rightarrow 2: 1$ hexanes/Et $2 \mathrm{O}$, gradient elution) to afford rubiginosin E (11) as a light brown oil (234 mg, 56\%). Data for rubiginosin E (11) matched that previously reported. ${ }^{4}$

\section{Data for rubiginosin E (11):}

$\mathbf{R}_{\mathbf{f}}: 0.20(2: 1$ hexanes/Et $2 \mathrm{O})$.

FTIR (neat): 3365, 2972, 2920, 1676, 1621, 1579, 1445, 1328, 1280, 1204, 1140, 1097, $1073 \mathrm{~cm}^{-1}$.

${ }^{1}$ H NMR (500 MHz, CDCl $)$ ): $\delta 6.63(\mathrm{~d}, J=10.0 \mathrm{~Hz}, 1 \mathrm{H}), 6.18$ (br s, 1H), 6.14 (s, 1H), 6.08 (s, 1H), $5.46(\mathrm{~d}, J=10.0 \mathrm{~Hz}, 1 \mathrm{H}), 5.29$ (t, $J=7.2 \mathrm{~Hz}, 1 \mathrm{H}), 3.02(\mathrm{~s}, 2 \mathrm{H}), 2.19-2.14(\mathrm{~m}, 2 \mathrm{H}), 2.16(\mathrm{~s}, 3 \mathrm{H}), 2.11$ $(\mathrm{s}, 3 \mathrm{H}), 1.88(\mathrm{~s}, 3 \mathrm{H}), 1.73-1.64(\mathrm{~m}, 2 \mathrm{H}), 1.56(\mathrm{~s}, 3 \mathrm{H}), 1.33(\mathrm{~s}, 3 \mathrm{H}) \mathrm{ppm}$.

${ }^{13}$ C NMR (150 MHz, CD ${ }_{3}$ OD): $\delta$ 201.9, 157.8, 155.2, 154.2, 140.4, 130.7, 130.6, 127.1, 123.9, 118.8, $109.3,109.2,108.1,78.9,56.0,41.7,27.7,26.6,23.9,21.7,20.8,16.3$ ppm.

HRMS (ESI): m/z: $[\mathrm{M}+\mathrm{H}]^{+}$Calcd for $\mathrm{C}_{22} \mathrm{H}_{29} \mathrm{O}_{3} 341.2111$; found 341.2110. 


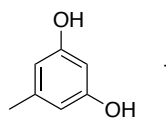

13: orcinol

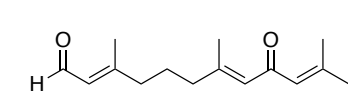

$E-19$

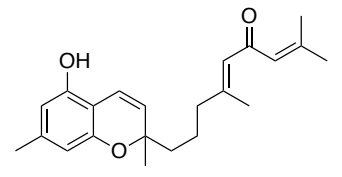

12: rubiginosin $D$

To a solution of aldehyde $\boldsymbol{E}-19$ (50 mg, $0.213 \mathrm{mmol}, 1.0$ equiv.) in PhMe (8 mL) was added orcinol monohydrate 13 ( $40 \mathrm{mg}, 0.320 \mathrm{mmol}, 1.5$ equiv.), and EDDA ( $4 \mathrm{mg}, 0.021 \mathrm{mmol}, 0.10$ equiv.). The mixture was heated to reflux using an aluminium heating block for $2 \mathrm{~h}$, and the solvent was removed in vacuo. The residue was purified by flash column chromatography on a Biotage ${ }^{\circledR}$ Isolera $^{\mathrm{TM}}$ 1.2.1 $(4: 1 \rightarrow$ 2:1 hexanes/ $\mathrm{Et}_{2} \mathrm{O}$, gradient elution) to afford rubiginosin $\mathrm{D}(\mathbf{1 2})$ as a light brown oil (48 $\mathrm{mg}, 66 \%$ ). Data for rubiginosin D (12) matched that previously reported. ${ }^{4}$

\section{Data for rubiginosin D (12):}

$\mathbf{R}_{\mathbf{f}}: 0.20(2: 1$ hexanes/Et $2 \mathrm{O})$.

FTIR (neat): 3360, 2923, 1620, 1444, 1330, 1218, 1097, 992, 824, $775 \mathrm{~cm}^{-1}$.

${ }^{1}$ H NMR (600 MHz, CD $\left.30 D\right): \delta 6.64(\mathrm{dd}, J=10.0,0.7 \mathrm{~Hz}, 1 \mathrm{H}), 6.14(\mathrm{~s}, 1 \mathrm{H}), 6.10-6.09(\mathrm{~m}, 1 \mathrm{H})$, $6.07(\mathrm{~s}, 1 \mathrm{H}), 6.06-6.05(\mathrm{~m}, 1 \mathrm{H}), 5.45(\mathrm{~d}, J=10.0 \mathrm{~Hz}, 1 \mathrm{H}), 2.16(\mathrm{~s}, 3 \mathrm{H}), 2.16-2.13(\mathrm{~m}, 2 \mathrm{H}), 2.12$ (s, $3 \mathrm{H}), 2.08(\mathrm{~s}, 3 \mathrm{H}), 1.90(\mathrm{~s}, 3 \mathrm{H}), 1.63-1.62(\mathrm{~m}, 4 \mathrm{H}), 1.32(\mathrm{~s}, 3 \mathrm{H}) \mathrm{ppm}$.

${ }^{13}$ C NMR (150 MHz, CD 3 OD): $\delta$ 193.8, 159.5, 156.2, 155.2, 154.2, 140.4, 127.3, 127.1, 126.8, 118.9, $109.3,109.2,108.1,78.9,42.2,41.4,27.8,26.6,22.9,21.7,20.8,19.3$ ppm.

HRMS (ESI): $\mathrm{m} / \mathrm{z}:[\mathrm{M}+\mathrm{H}]^{+}$Calcd for $\mathrm{C}_{22} \mathrm{H}_{29} \mathrm{O}_{3} 341.2111$; found 341.2116. 


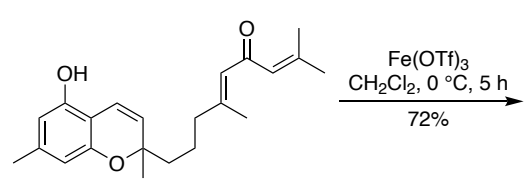

12: rubiginosin $D$

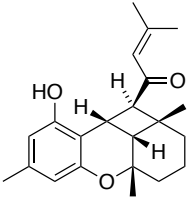

4: rubiginosin $\mathrm{A}$

To a solution of rubiginosin $\mathrm{D}$ (12) (46 mg, $0.135 \mathrm{mmol}, 1.0$ equiv.) in $\mathrm{CH}_{2} \mathrm{Cl}_{2}(5 \mathrm{~mL})$ at $0{ }^{\circ} \mathrm{C}$ was added $\mathrm{Fe}(\mathrm{OTf})_{3}(20 \mathrm{mg}, 0.040 \mathrm{mmol}, 0.3$ equiv.) and the reaction was left to slowly warm to room temperature over $5 \mathrm{~h}$. Then quenched upon addition of brine $(10 \mathrm{~mL})$ and the products extracted with $\mathrm{CH}_{2} \mathrm{Cl}_{2}(3 \times 10 \mathrm{~mL})$. The combined organic layers were then dried with $\mathrm{MgSO}_{4}$, filtered and concentrated in vacuo. Purification by flash column chromatography on $\mathrm{SiO}_{2}$ (19:1 hexanes/EtOAc) then afforded rubiginosin A (4) (33 mg, 72\%). Data for rubiginosin A (4) matched that previously reported in literature. ${ }^{4}$ 


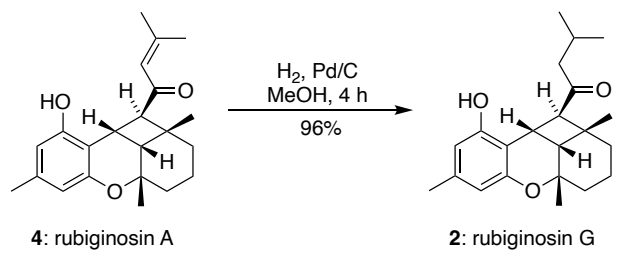

To a solution of rubiginosin A (4) (30 mg, $0.059 \mathrm{mmol}, 1.0$ equiv.) in dry $\mathrm{MeOH}(15 \mathrm{~mL})$ was added portion-wise $\mathrm{Pd} / \mathrm{C}(55 \mathrm{mg}, 0.005 \mathrm{mmol}, 10 \% \mathrm{w} / \mathrm{w}, 0.10$ equiv.). The solution was then put under vacuum/ flushed with $\mathrm{H}_{2}$ three times and the reaction was left to stir at room temperature for $4 \mathrm{~h}$. The reaction was then filtered through celite with EtOAc and concentrated to afford rubiginosin G (2) (29 mg, 96\%) as a clear oil. Data for rubiginosin G (2) matched that previously reported. ${ }^{4}$

\section{Data for rubiginosin G (2):}

$\mathbf{R}_{\mathbf{f}}: 0.20$ (19:1 hexanes/EtOAc).

FTIR (neat): 3395, 2956, 2929, 1682, 1631, 1585, 1456, 1370, 1323, 1260, 1127, 1059, $908 \mathrm{~cm}^{-1}$.

${ }^{1}$ H NMR (600 MHz, CDCl $): \delta 6.83(\mathrm{~s}, 1 \mathrm{H}), 6.40(\mathrm{~s}, 1 \mathrm{H}), 6.31(\mathrm{~s}, 1 \mathrm{H}), 3.81(\mathrm{t}, J=9.4 \mathrm{~Hz}, 1 \mathrm{H}), 2.91$ (d, $J=9.4 \mathrm{~Hz}, 1 \mathrm{H}), 2.23$ (s, 3H), $2.18(\mathrm{dd}, J=13.5,6.6 \mathrm{~Hz}, 1 \mathrm{H}), 2.15-2.10$ (m, 1H), $2.09-2.05$ (m, 1H), $2.00-1.91(\mathrm{~m}, 1 \mathrm{H}), 1.87-1.81(\mathrm{~m}, 1 \mathrm{H}), 1.80(\mathrm{~d}, J=9.3 \mathrm{~Hz}, 1 \mathrm{H}), 1.70-1.63(\mathrm{~m}), 1.37-1.33$ (m, 1H), $1.33-1.28(\mathrm{~m}, 1 \mathrm{H}), 1.17(\mathrm{~s}, 3 \mathrm{H}), 1.11(\mathrm{~s}, 3 \mathrm{H}), 0.89$ (d, $J=6.4 \mathrm{~Hz}, 3 \mathrm{H}), 0.86(\mathrm{~d}, J=6.4 \mathrm{~Hz}$, 3H) $\mathrm{ppm}$.

${ }^{13}$ C NMR (150 MHz, $\left.\mathbf{C D C l}_{3}\right): \delta 213.9,154.5,153.1,138.1,111.8,111.7,110.6,75.3,57.4,50.9$, $45.2,39.4,36.6,34.5,29.6,25.8,24.2,23.9,23.0,22.8,21.6,17.3 \mathrm{ppm}$.

HRMS (ESI): m/z: [M+Na] $]^{+}$Calcd for $\mathrm{C}_{22} \mathrm{H}_{30} \mathrm{O}_{3} \mathrm{Na}$ 365.2087; found 365.2082. 


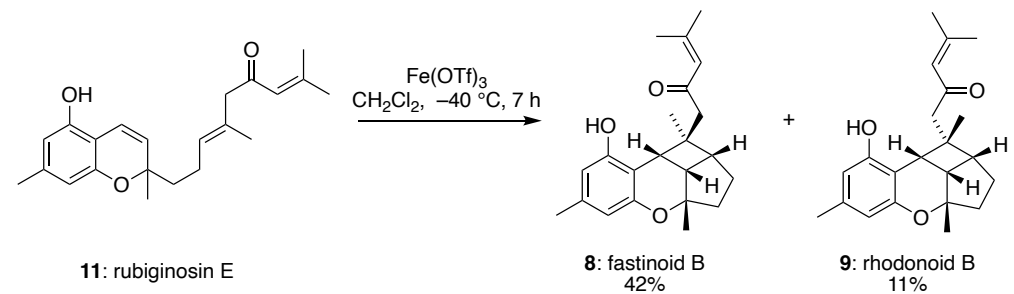

To a solution of rubiginosin $\mathrm{E}$ (11) $\left(53 \mathrm{mg}, 0.156 \mathrm{mmol}, 1.0\right.$ equiv.) in $\mathrm{CH}_{2} \mathrm{Cl}_{2}(10 \mathrm{~mL})$ at $-40{ }^{\circ} \mathrm{C}$ was added $\mathrm{Fe}(\mathrm{OTf})_{3}(23 \mathrm{mg}, 0.047 \mathrm{mmol}, 0.3$ equiv.) and the reaction was left to slowly warm to room temperature over $7 \mathrm{~h}$. Then quenched upon addition of brine $(10 \mathrm{~mL})$ and the products extracted with $\mathrm{CH}_{2} \mathrm{Cl}_{2}$ (3 x $10 \mathrm{~mL}$ ). The combined organic layers were then dried with $\mathrm{MgSO}_{4}$, filtered and concentrated in vacuo. Purification by flash column chromatography on $\mathrm{SiO}_{2}(20: 1$ hexanes/EtOAc) then afforded fastinoid B (8) as a white solid (22 mg, 42\%) and rhodonoid B (9) (6 mg, 11\%) as a white solid. Data for fastinoid B (8) and rhodonoid B (9) matched that previously reported in literature. ${ }^{5,6}$

\section{Data for fastinoid B (8):}

$\mathbf{R}_{\mathbf{f}}: 0.25$ (20:1 hexanes/EtOAc).

MP: $110.4-112.0^{\circ} \mathrm{C}$.

FTIR (neat): 3250, 2947, 1678, 1613, 1574, 1490, 1444, 1376, 1177, 1057, 910, 827, $732 \mathrm{~cm}^{-1}$.

${ }^{1}$ H NMR (500 MHz, CDCl $)$ ): $\delta 9.25(\mathrm{~s}, 1 \mathrm{H}), 6.39(\mathrm{~s}, 1 \mathrm{H}), 6.27(\mathrm{~s}, 1 \mathrm{H}), 6.11(\mathrm{~s}, 1 \mathrm{H}), 3.38(\mathrm{~d}, J=9.6$ $\mathrm{Hz}, 1 \mathrm{H}), 2.97(\mathrm{~d}, J=17.7 \mathrm{~Hz}, 1 \mathrm{H}), 2.79(\mathrm{~d}, J=17.7 \mathrm{~Hz}, 1 \mathrm{H}), 2.68(\mathrm{t}, J=8.8 \mathrm{~Hz}, 1 \mathrm{H}), 2.31(\mathrm{td}, J=8.4$, $2.9 \mathrm{~Hz}, 1 \mathrm{H}), 2.23(\mathrm{~s}, 3 \mathrm{H}), 2.20(\mathrm{~s}, 3 \mathrm{H}), 2.08-2.02(\mathrm{~m}, 1 \mathrm{H}), 1.92(\mathrm{~s}, 3 \mathrm{H}), 1.80-1.73(\mathrm{~m}, 1 \mathrm{H}), 1.66-$ $1.59(\mathrm{~m}, 1 \mathrm{H}), 1.56-1.51(\mathrm{~m}, 1 \mathrm{H}), 1.31(\mathrm{~s}, 3 \mathrm{H}), 0.84(\mathrm{~s}, 3 \mathrm{H}) \mathrm{ppm}$.

${ }^{13}$ C NMR (150 MHz, CDCl $): \delta 203.1,159.6,156.2,154.6,138.2,124.1,110.8,110.4,108.0,83.0$, $58.7,46.9,41.7,40.6,38.5,34.2,28.2,26.3,24.8,21.5,21.3,15.5$ ppm.

HRMS (ESI): m/z: [M+H] $]^{+}$Calcd for $\mathrm{C}_{22} \mathrm{H}_{29} \mathrm{O}_{3} 341.2111$; found 341.2115.

\section{Data for rhodonoid B (9):}

$\mathbf{R}_{\mathbf{f}}: 0.20$ (20:1 hexanes/EtOAc).

MP: $145.3-149.8^{\circ} \mathrm{C}\left(\right.$ lit. $\left.148-149^{\circ} \mathrm{C}\right) .^{6}$

FTIR (neat): 3395, 2949, 1677, 1619, 1585, 1444, 1418, 1328, 1139, 1054, 907, 825, $732 \mathrm{~cm}^{-1}$.

${ }^{1}$ H NMR (500 MHz, CDCl $)$ ): $\delta 6.33(\mathrm{~s}, 1 \mathrm{H}), 6.25(\mathrm{~s}, 1 \mathrm{H}), 5.89-5.88(\mathrm{~m}, 1 \mathrm{H}), 5.35(\mathrm{~s}, 1 \mathrm{H}), 3.18(\mathrm{~d}, J$ $=9.3 \mathrm{~Hz}, 1 \mathrm{H}), 2.62(\mathrm{td}, J=8.0,2.6 \mathrm{~Hz}), 2.60(\mathrm{dd}, J=9.0,8.0 \mathrm{~Hz}, 1 \mathrm{H}), 2.47(\mathrm{~d}, J=18.3 \mathrm{~Hz}, 1 \mathrm{H}), 2.42$ (d, $J=18.3 \mathrm{~Hz}, 1 \mathrm{H}), 2.22(\mathrm{~s}, 3 \mathrm{H}), 2.04(\mathrm{~s}, 3 \mathrm{H}), 1.98-1.90(\mathrm{~m}, 1 \mathrm{H}), 1.78(\mathrm{~s}, 3 \mathrm{H}), 1.75-1.68(\mathrm{~m}, 1 \mathrm{H})$, $1.66-1.61(\mathrm{~m}, 1 \mathrm{H}), 1.60-1.56(\mathrm{~m}, 1 \mathrm{H}), 1.54(\mathrm{~s}, 3 \mathrm{H}), 1.32(\mathrm{~s}, 3 \mathrm{H}) \mathrm{ppm}$.

${ }^{13} \mathbf{C}$ NMR (150 MHz, $\left.\mathbf{C D C l}_{3}\right): \delta 201.6,154.5,154.4,154.2,137.8,125.0,111.9,109.6,109.6,83.9$, 47.0, 45.6, 41.7, 39.5, 39.1, 36.5, 30.5, 27.7, 26.9, 26.4, 21.4, $20.7 \mathrm{ppm}$.

HRMS (ESI): $\mathrm{m} / \mathrm{z}:[\mathrm{M}+\mathrm{H}]^{+}$Calcd for $\mathrm{C}_{22} \mathrm{H}_{29} \mathrm{O}_{3} 341.2111$; found 341.2114.

\footnotetext{
${ }^{5}$ Huang, G.-H.; Lei, C.; Zhu, K.-X.; Li, J.-Y.; Li, J.; Hou, A.-J. Chin. J. Nat. Med. 2019, 17, 963.

${ }^{6}$ Liao, H.-B.; Lei, C.; Gao, L.-X.; Li, J.-Y.; Li, J.; Hou, A.-J. Org. Lett. 2015, 17, 5040.
} 


\section{NMR Spectra}

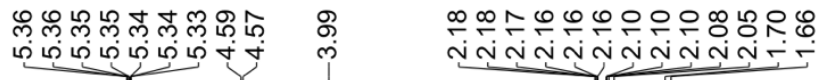

$\underset{500 \mathrm{MHz}}{{ }^{1} \mathrm{NDCl}_{3}}$

SI-1
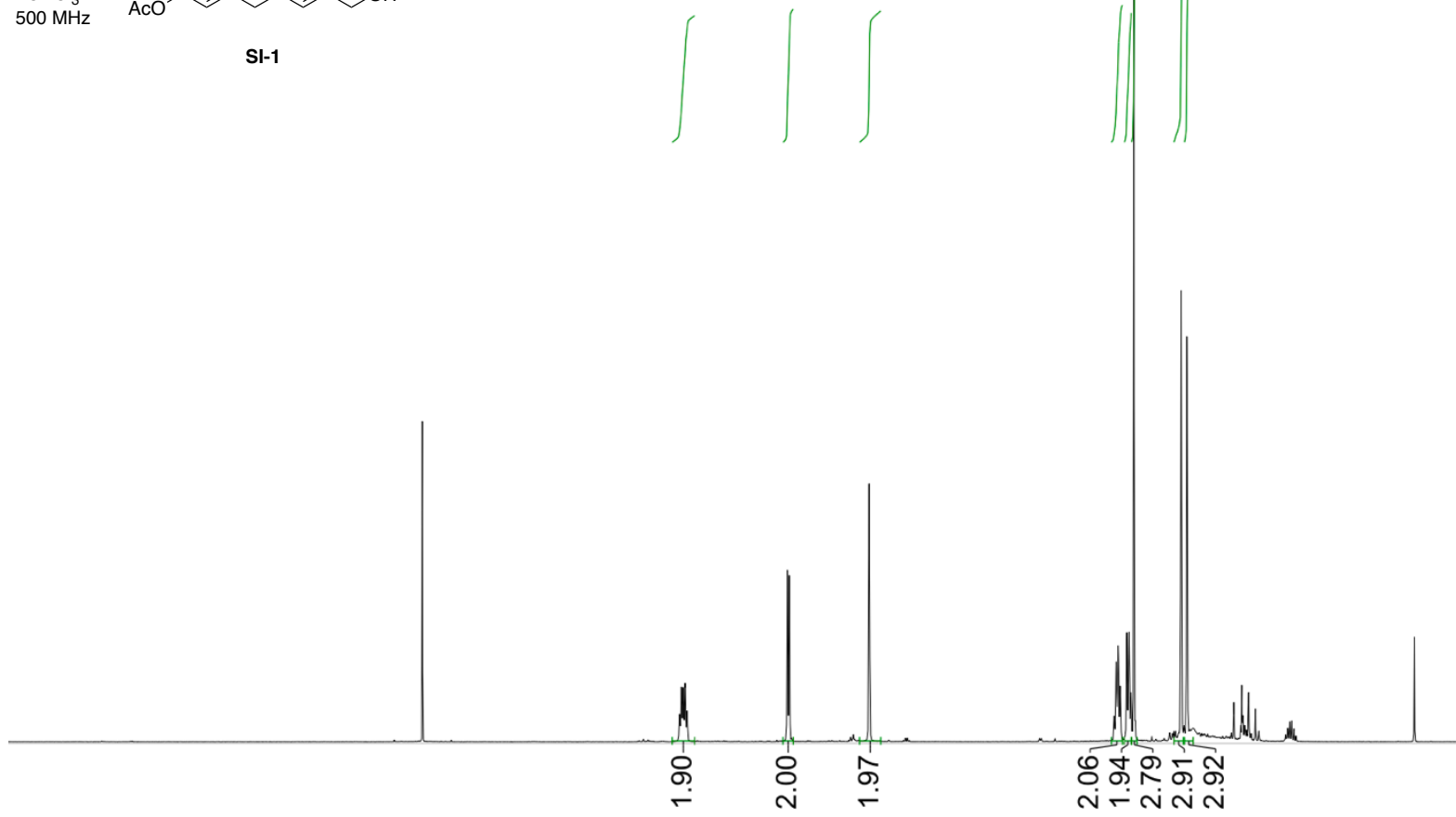

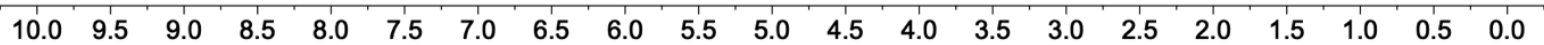

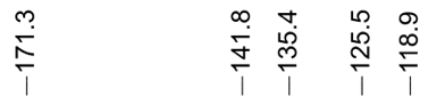

ఠே

尺ָ̣

ஸें ำ

${ }_{125 \mathrm{MHz}}^{{ }^{13} \mathrm{C} \mathrm{NMRl}_{3}}$

SI-1

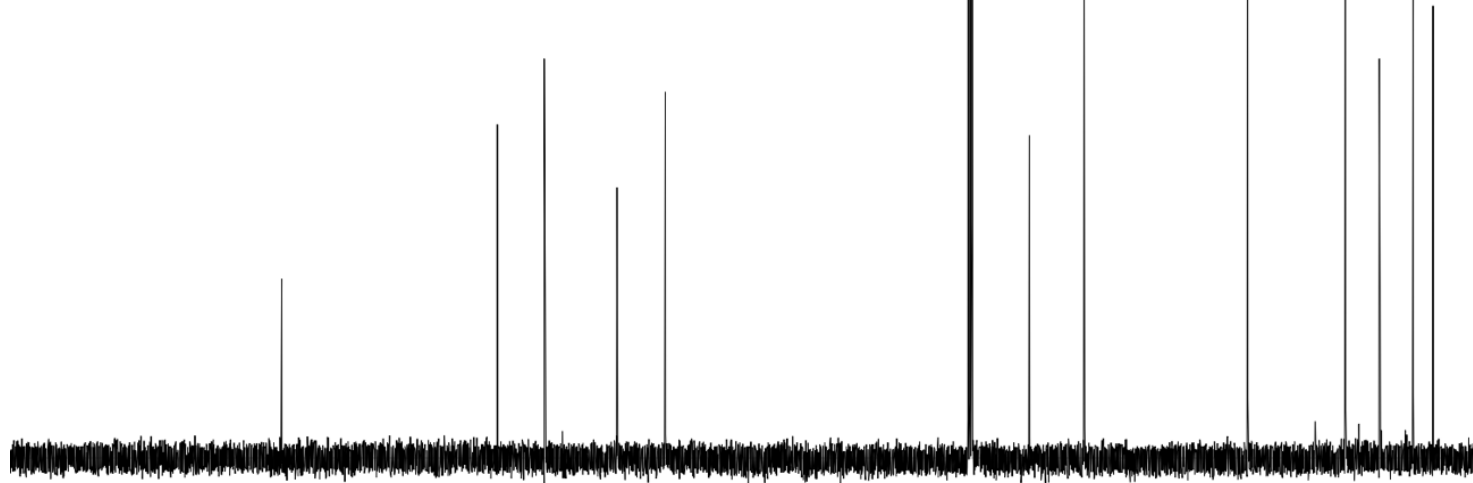

$\begin{array}{lllllllllllllllllllll}200 & 190 & 180 & 170 & 160 & 150 & 140 & 130 & 120 & 110 & 100 & 90 & 80 & 70 & 60 & 50 & 40 & 30 & 20 & 10 & 0\end{array}$ 
$\stackrel{\sharp}{m}$

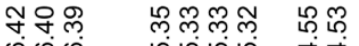

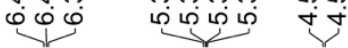

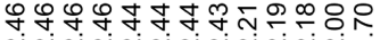

$\underset{H}{{ }_{500 \mathrm{MHz}} \mathrm{CDM}_{3}}$

SI-2
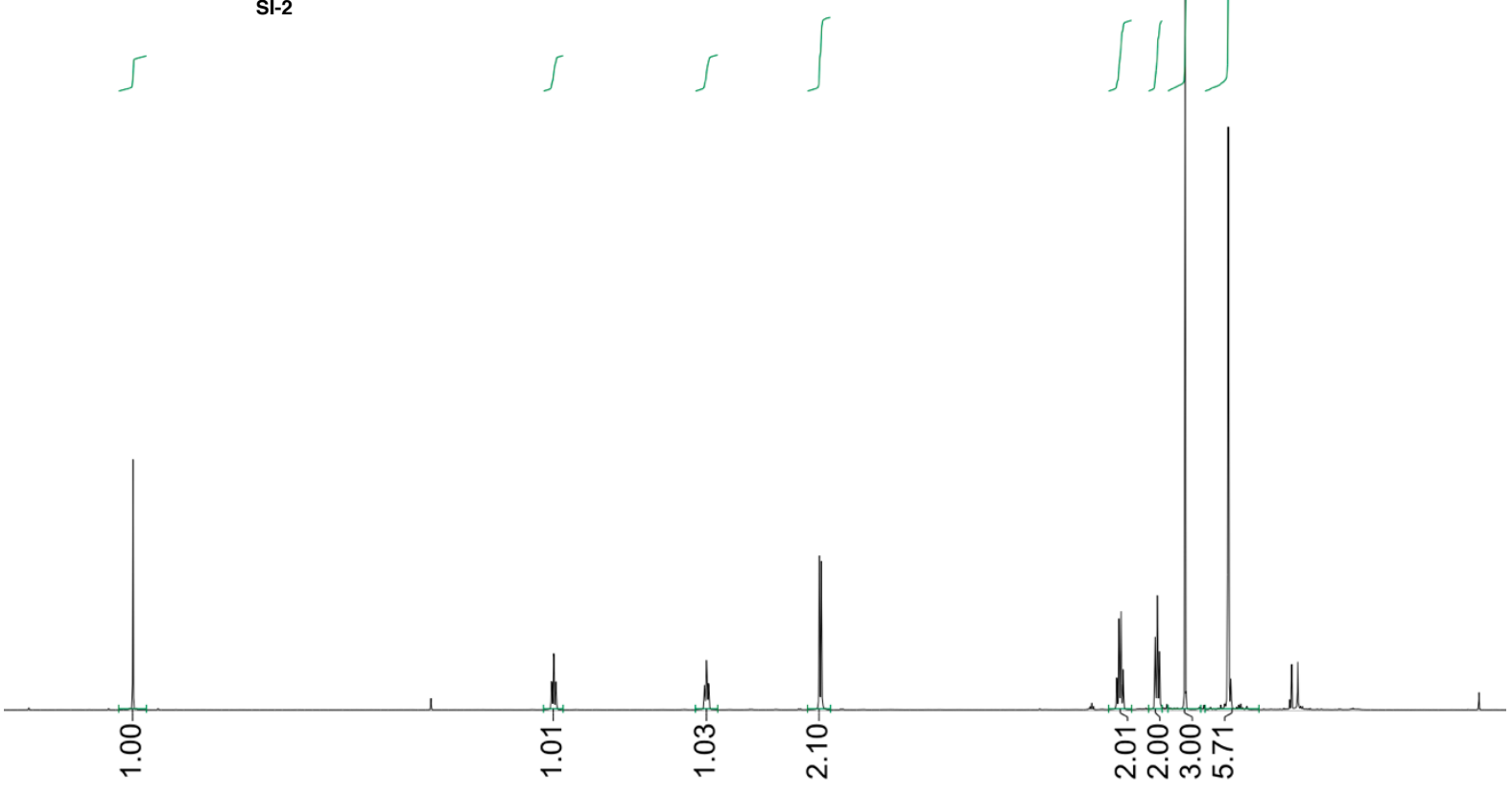

$\begin{array}{lllllllllllllllllllll}10.0 & 9.5 & 9.0 & 8.5 & 8.0 & 7.5 & 7.0 & 6.5 & 6.0 & 5.5 & 5.0 & 4.5 & 4.0 & 3.5 & 3.0 & 2.5 & 2.0 & 1.5 & 1.0 & 0.5 & 0.0\end{array}$

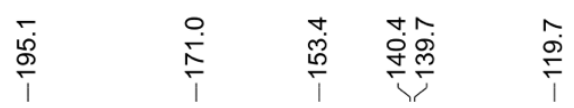

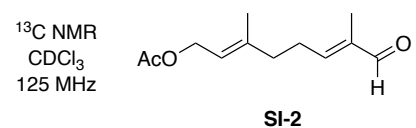

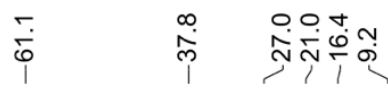

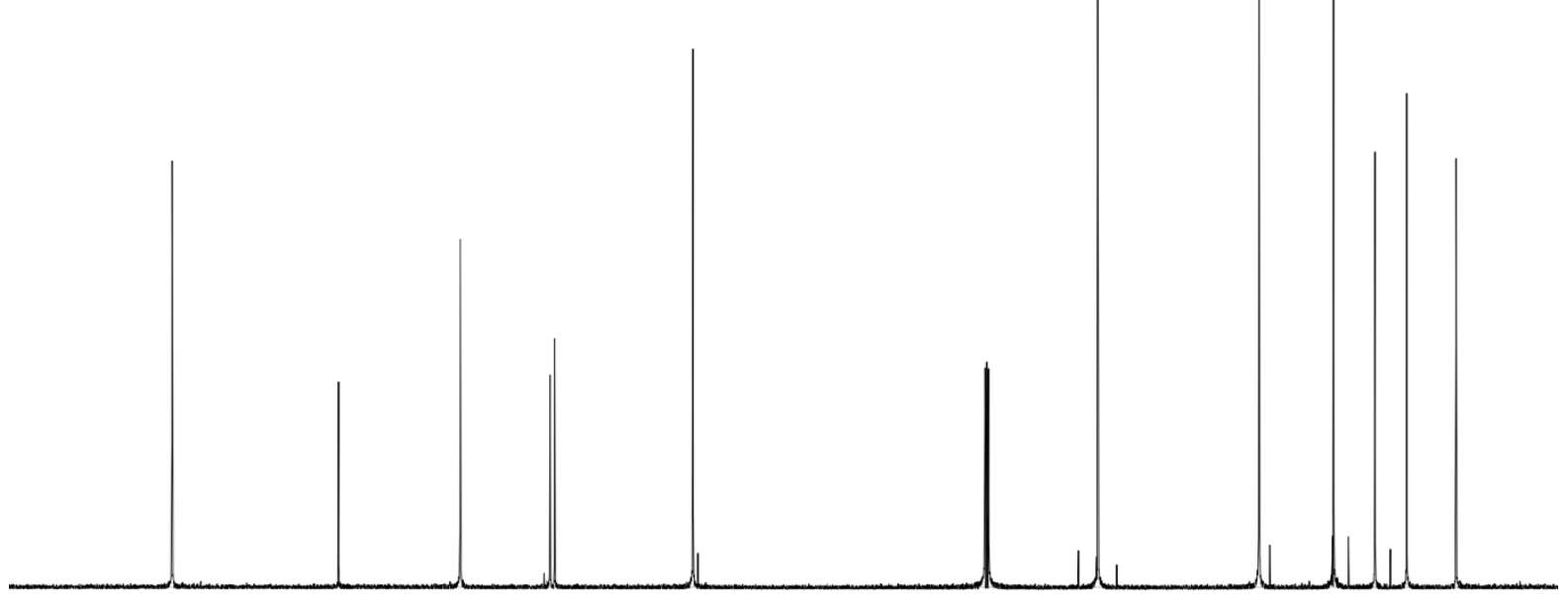

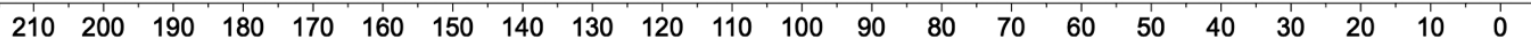




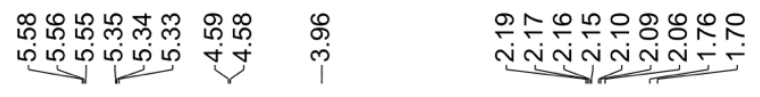

$\underset{500 \mathrm{MHz}}{{ }^{1} \mathrm{H} \mathrm{NMR}}$

15

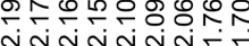
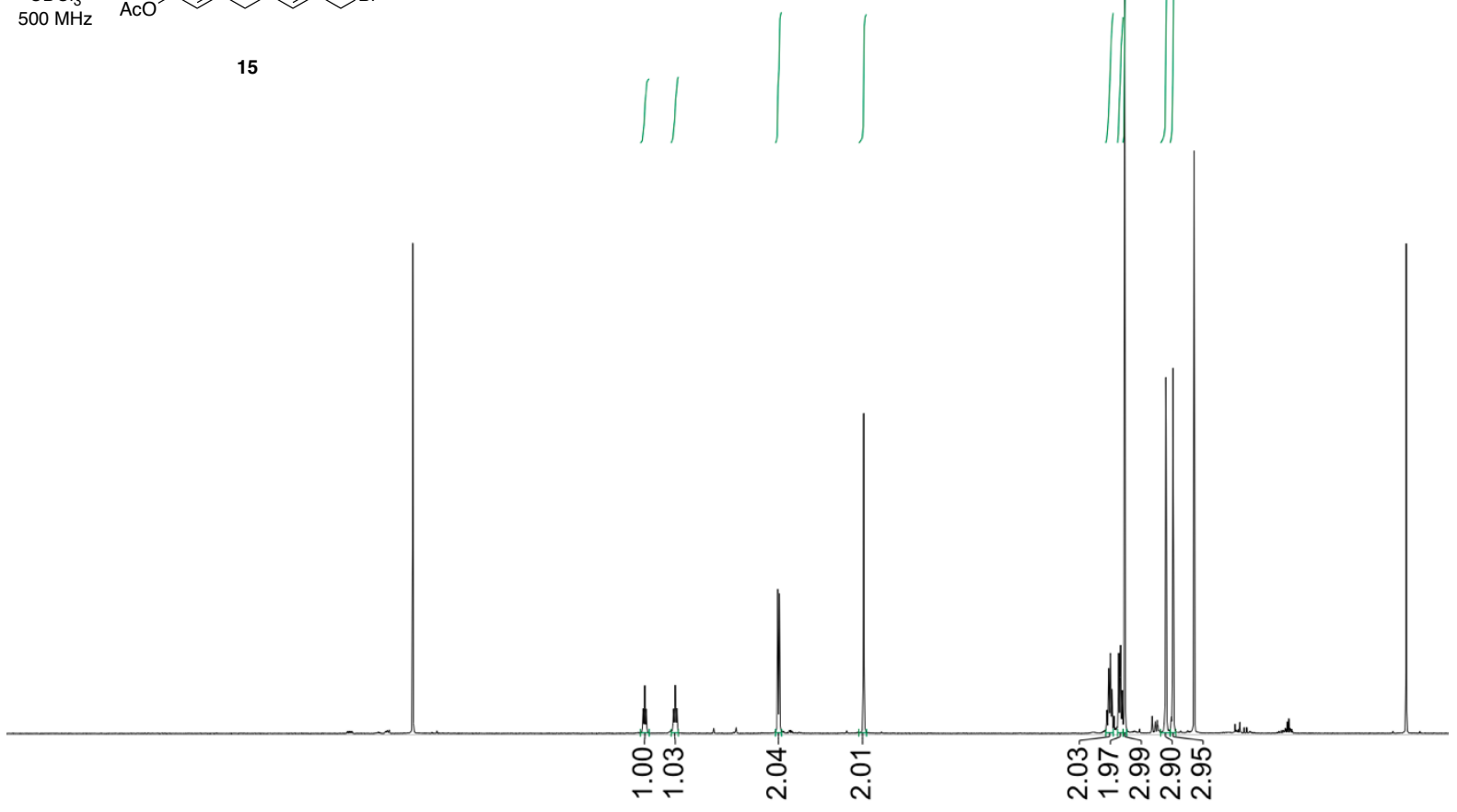

\begin{tabular}{llllllllllllllllllllllll}
\hline 10.0 & 9.5 & 9.0 & 8.5 & 8.0 & 7.5 & 7.0 & 6.5 & 6.0 & 5.5 & 5.0 & 4.5 & 4.0 & 3.5 & 3.0 & 2.5 & 2.0 & 1.5 & 1.0 & 0.5 & 0.0
\end{tabular}

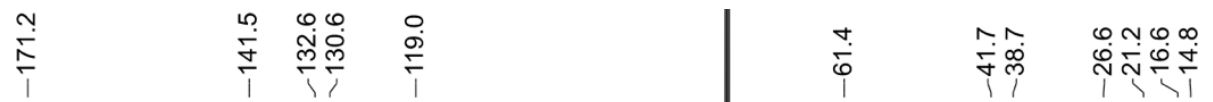

$\underset{125 \mathrm{MHz}}{{ }_{13}^{13} \mathrm{CNMR}_{3}}$

15

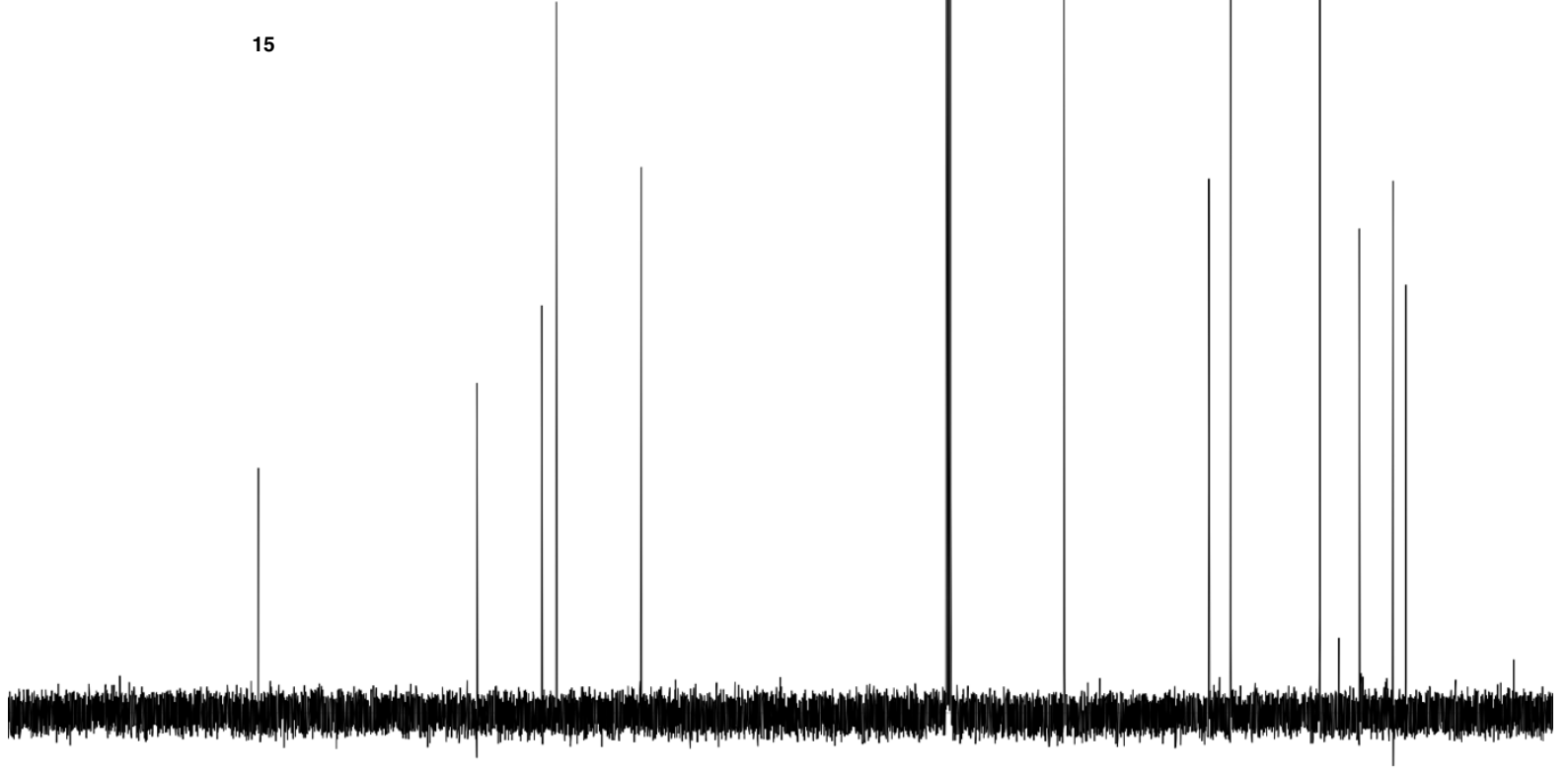

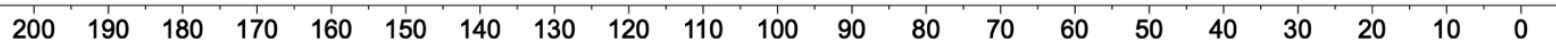




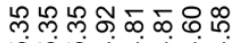

ம் ம்

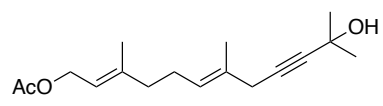

16

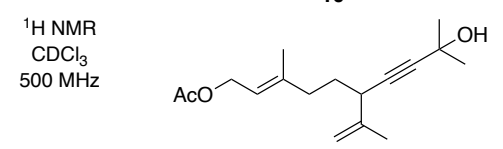

SI-3

16:SI-3 1.2:1

inseparable mixture

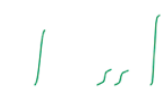

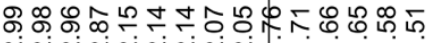
nัง nกง

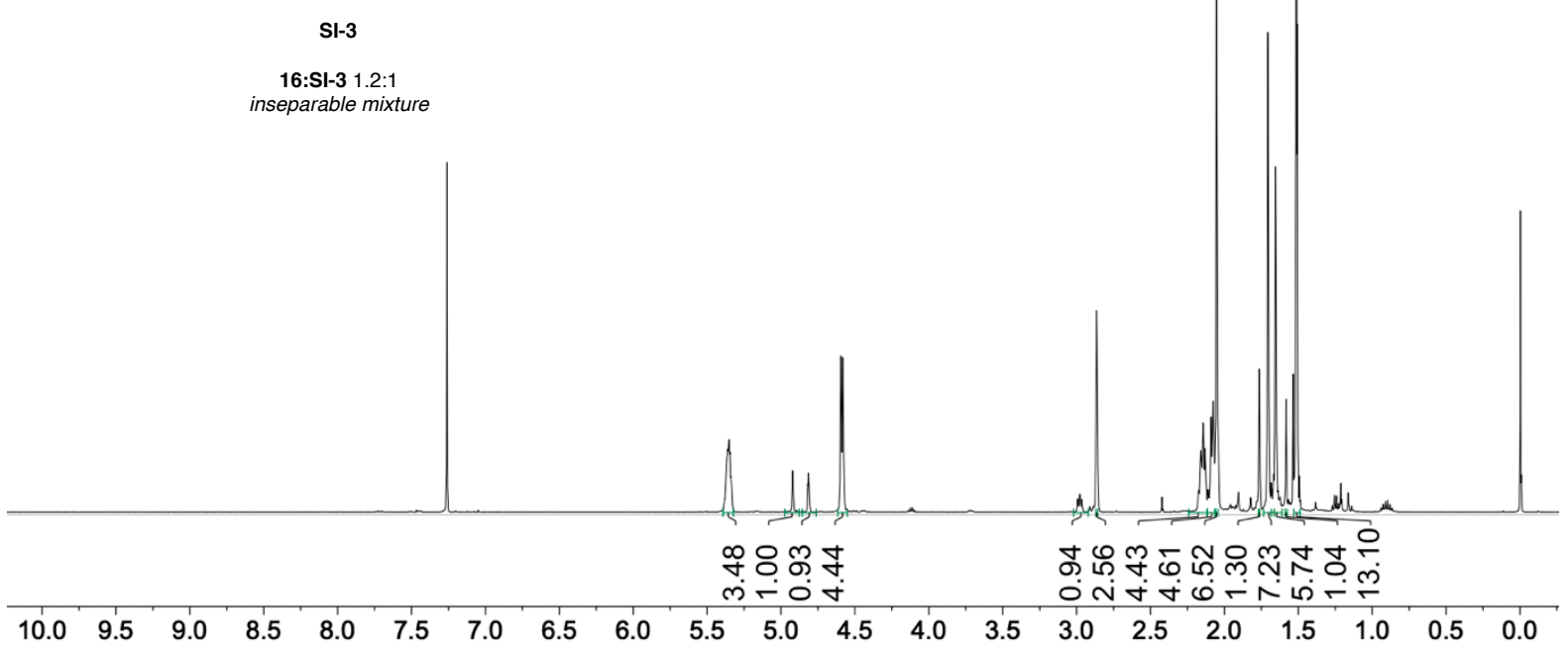

率

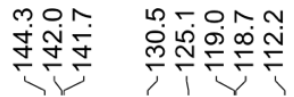

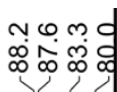

$\forall \varphi$ m

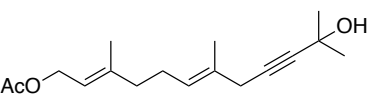

${ }^{13} \mathrm{C} \mathrm{NMR}_{\mathrm{CDCl}_{3}}$

$125 \mathrm{MHz}$

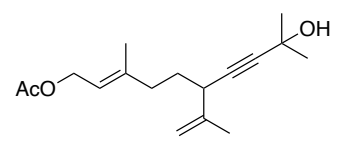

SI-3

16:SI-3 1.2:

inseparable mixture 
${ }^{1} \mathrm{H} \mathrm{NMR}$
$\mathrm{CDCl}_{3}$

17
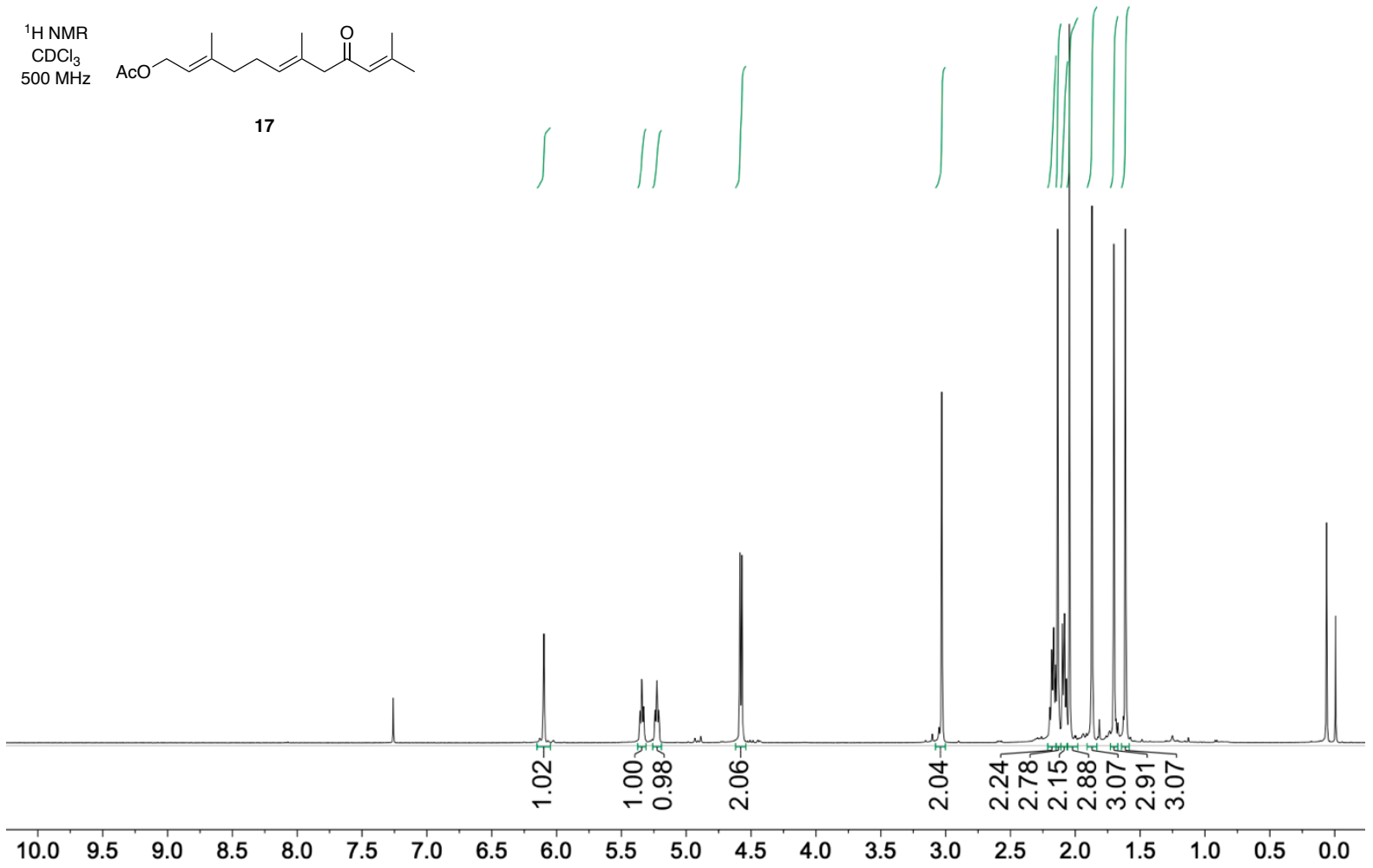

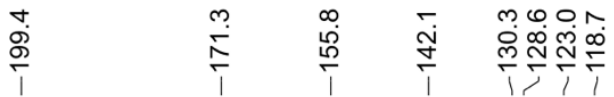

它

m.

${ }^{3} \mathrm{C}$ NMR

$25 \mathrm{MHz}$

17

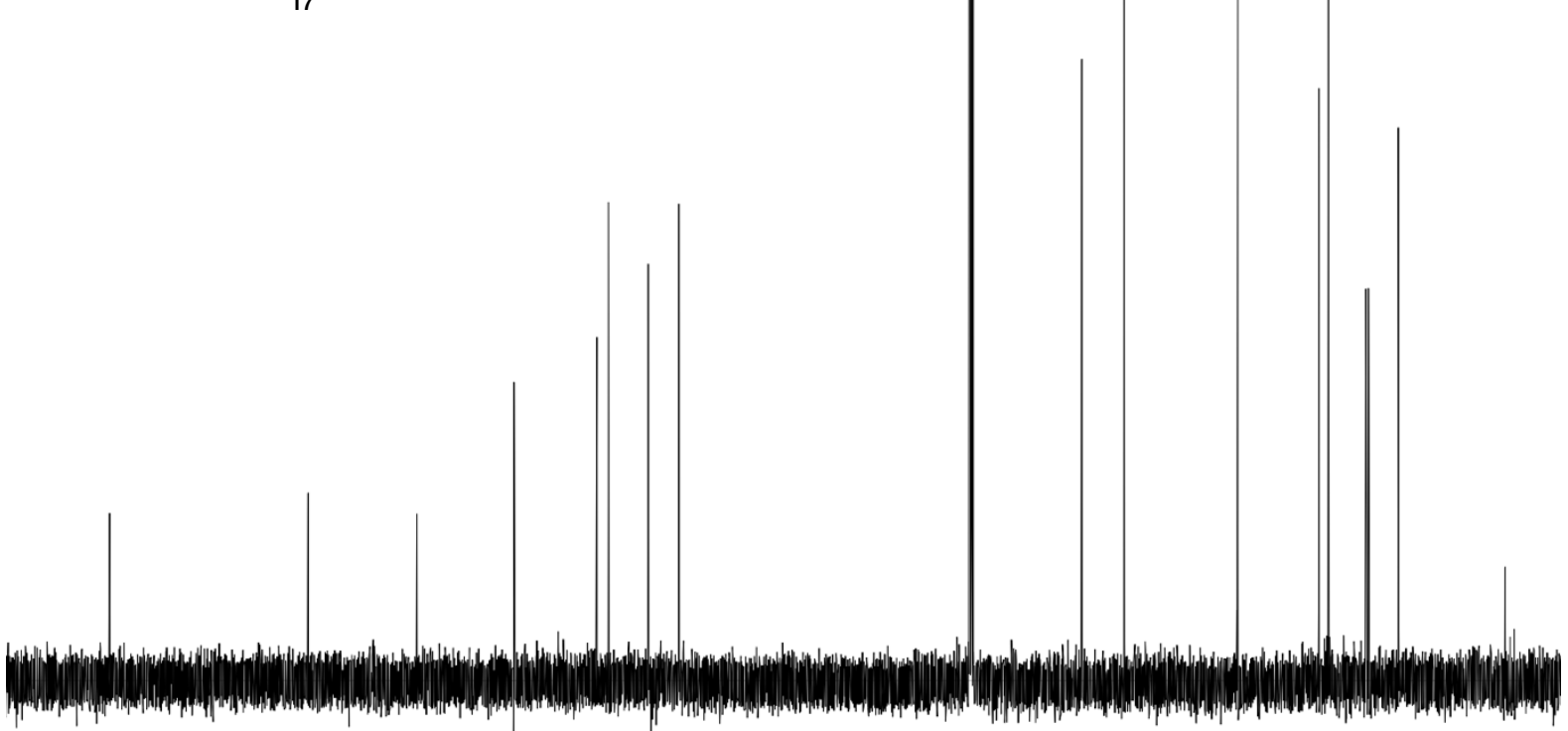

$\begin{array}{llllllllllllllllllllllll}210 & 200 & 190 & 180 & 170 & 160 & 150 & 140 & 130 & 120 & 110 & 100 & 90 & 80 & 70 & 60 & 50 & 40 & 30 & 20 & 10 & 0\end{array}$ 

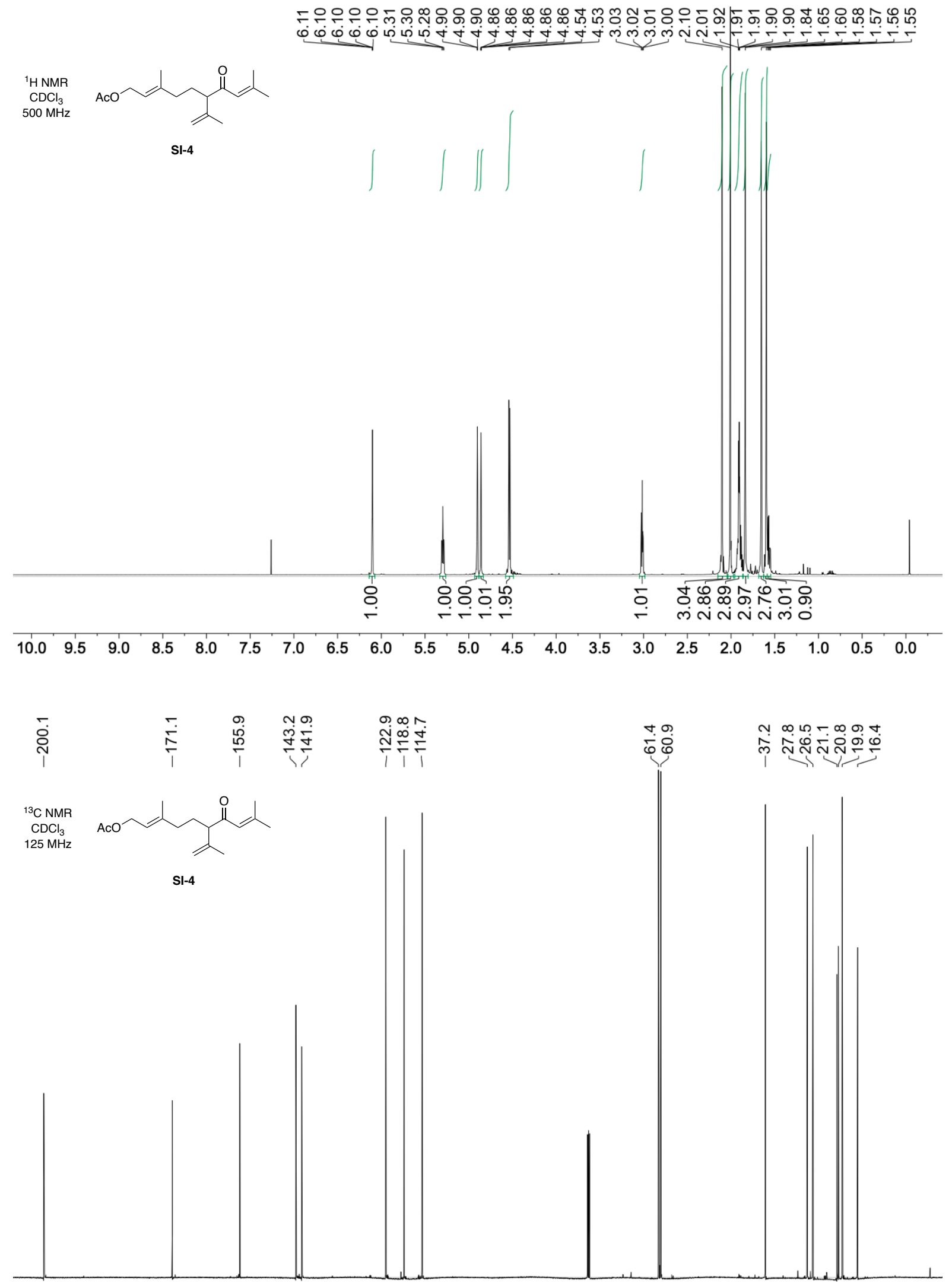

$\begin{array}{lllllllllllllllllllll}200 & 190 & 180 & 170 & 160 & 150 & 140 & 130 & 120 & 110 & 100 & 90 & 80 & 70 & 60 & 50 & 40 & 30 & 20 & 10 & 0\end{array}$ 
$\underset{500 \mathrm{MHz}}{{ }^{1} \mathrm{HNMR}_{3}} \mathrm{CDCl}_{3}$

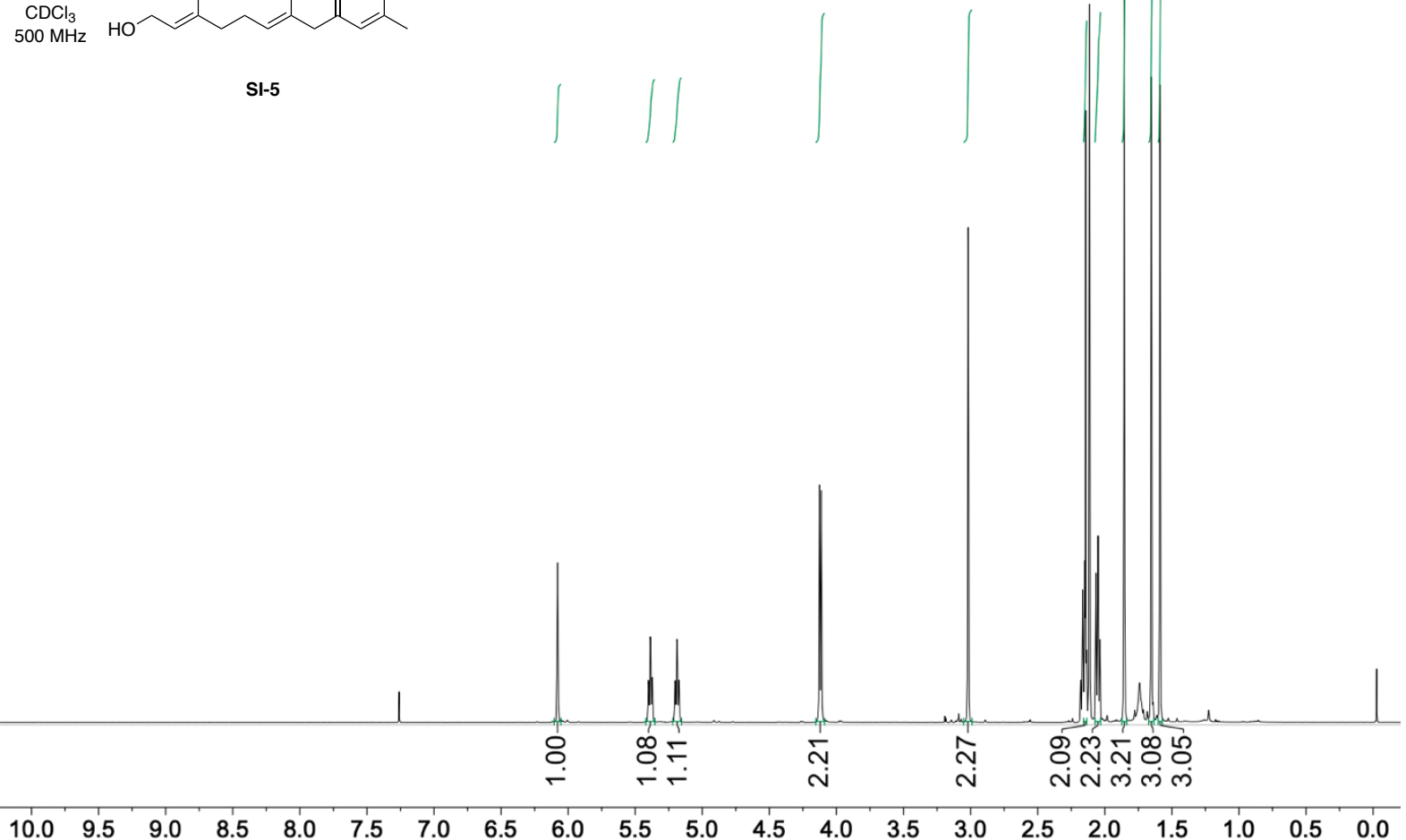

$\underset{125 \mathrm{MHz}}{\mathrm{CDCl}_{3}^{13} \mathrm{C} \mathrm{NMR}_{3}}$

SI-5

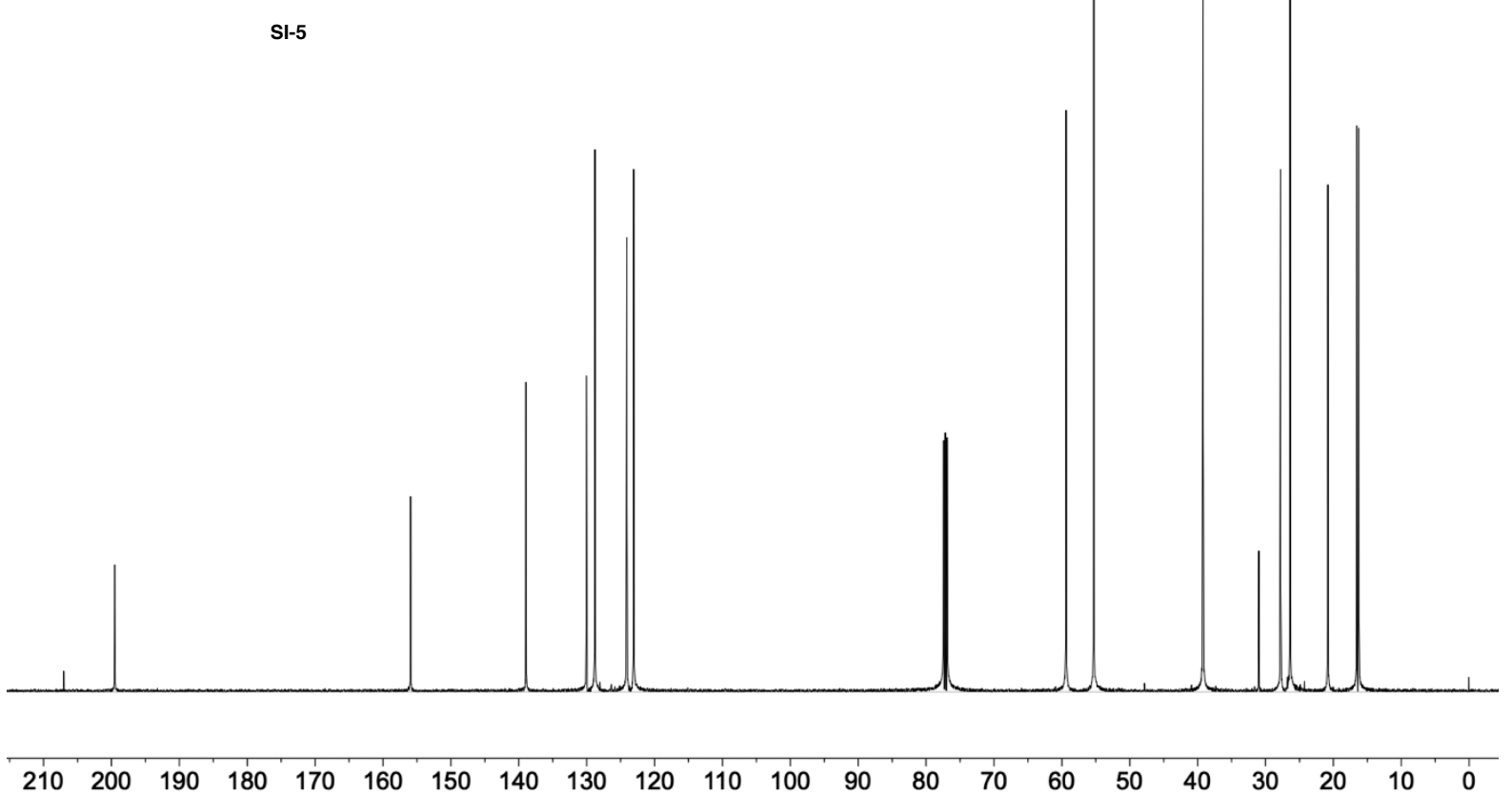



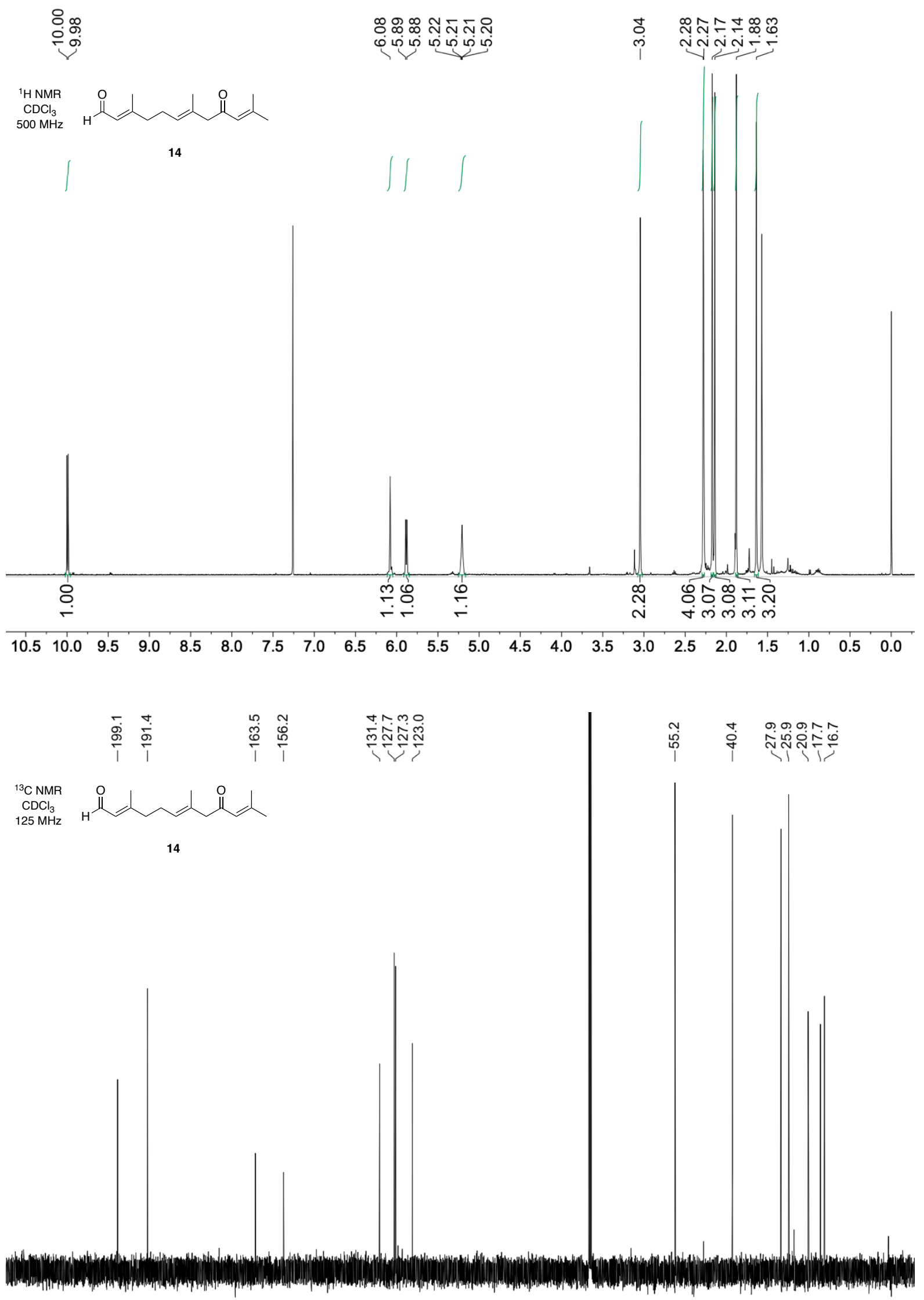

$\begin{array}{lllllllllllllllllllllllll}220 & 210 & 200 & 190 & 180 & 170 & 160 & 150 & 140 & 130 & 120 & 110 & 100 & 90 & 80 & 70 & 60 & 50 & 40 & 30 & 20 & 10 & 0\end{array}$ 


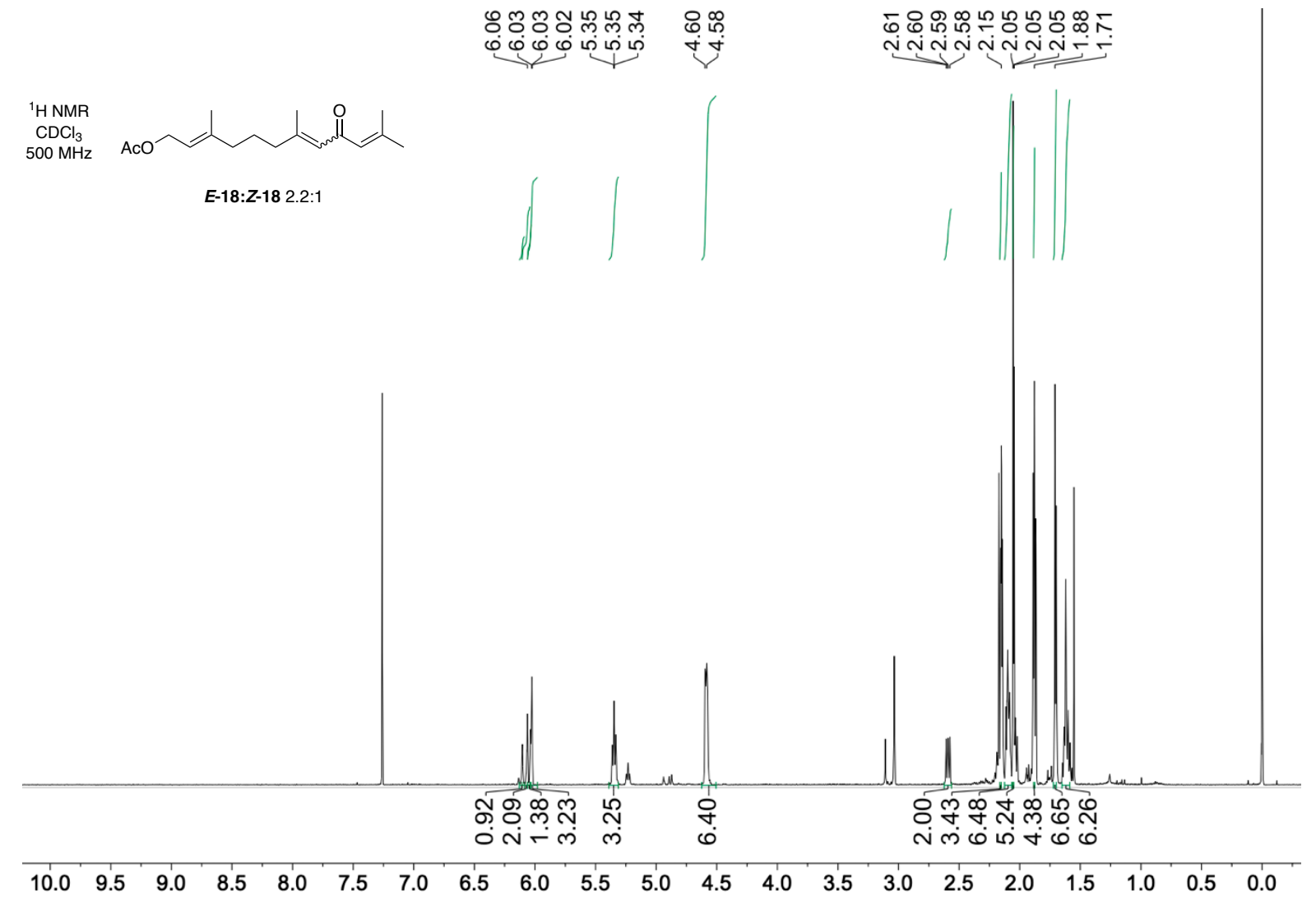

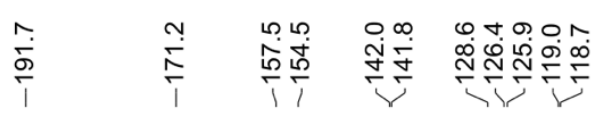

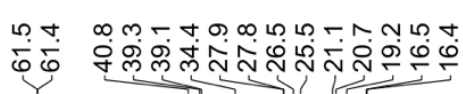

${ }^{13} \mathrm{C}$ NMR

$\mathrm{CDCl}_{3}$

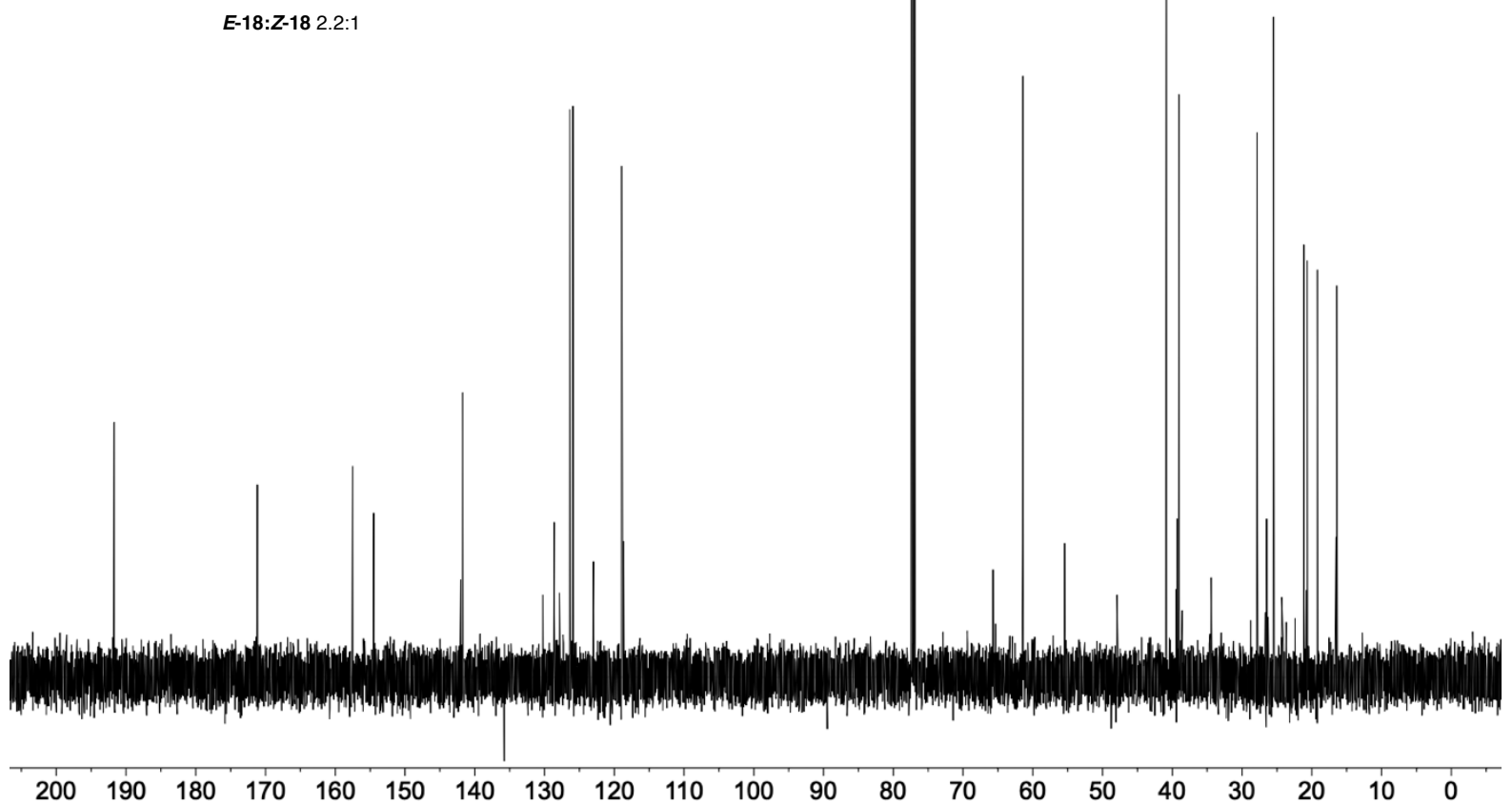


$\underset{500 \mathrm{MHz}}{\mathrm{CDCl}_{3}}$
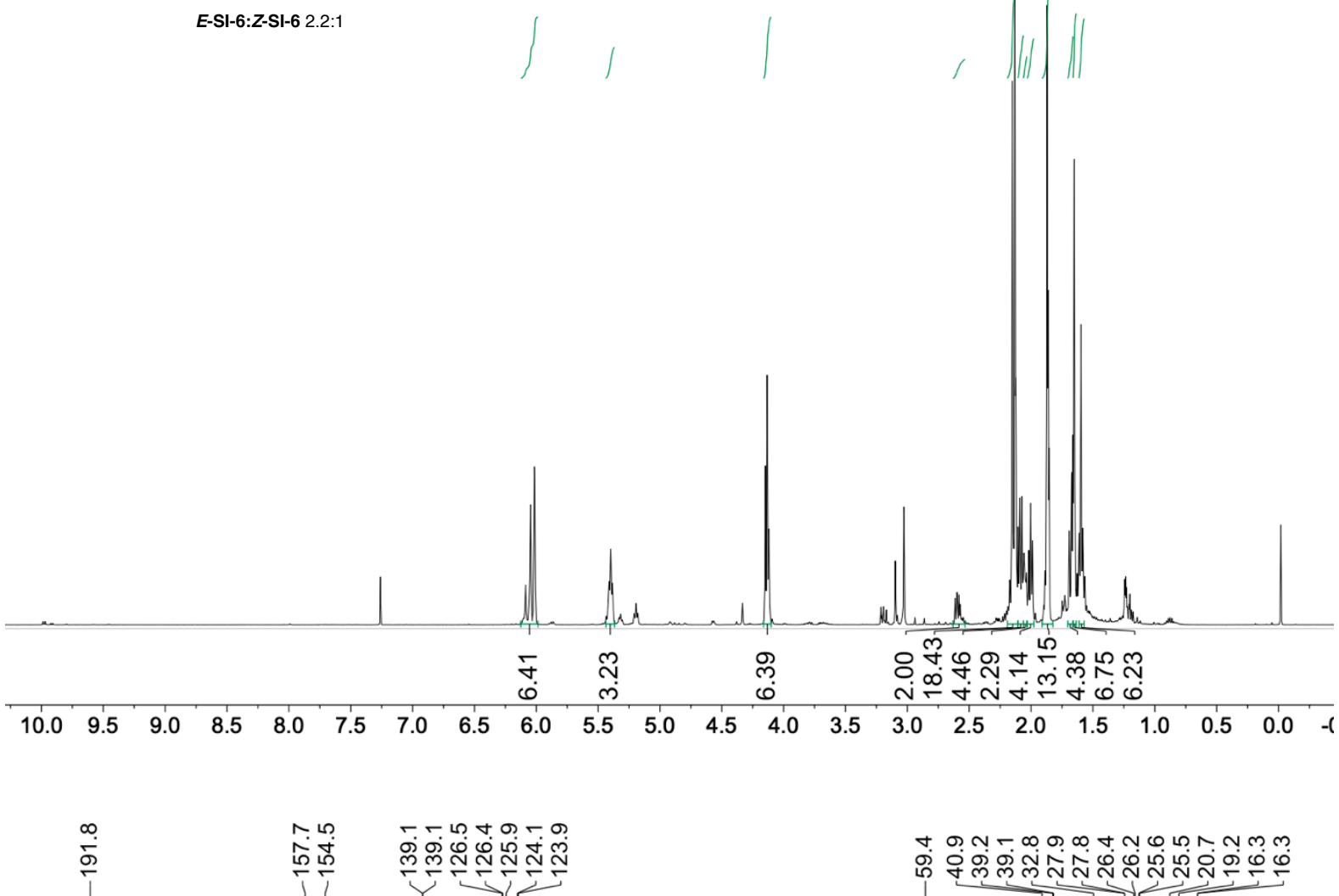

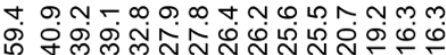

NMR

$\mathrm{CDCl}_{3}$

$125 \mathrm{MHz}$

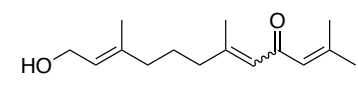

E-SI-6:Z-SI-6 2.2:1

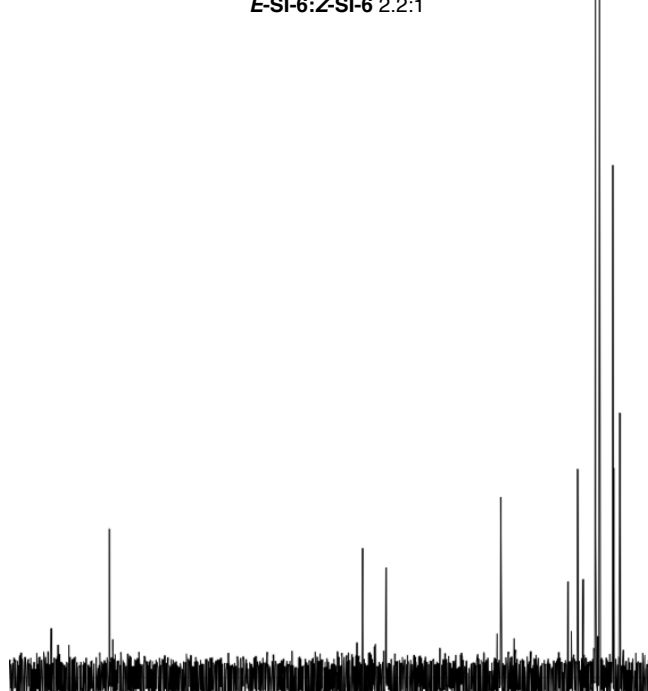

म

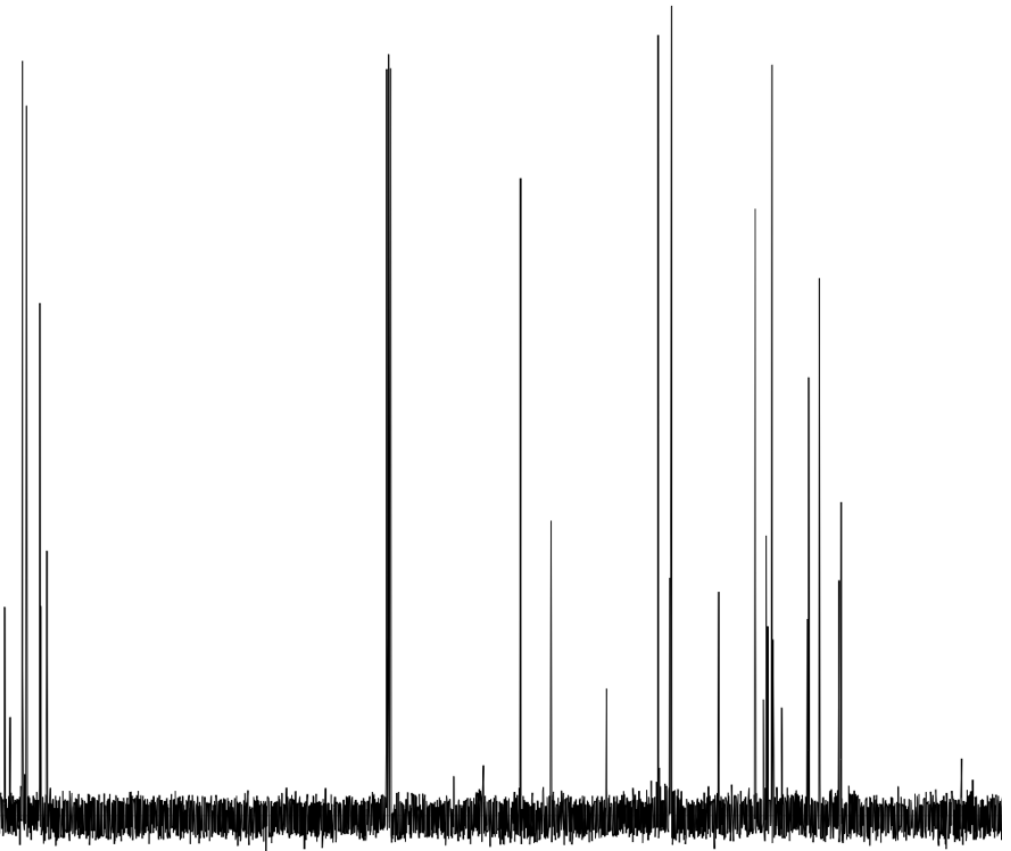

$\begin{array}{lllllllllllllllllllll}200 & 190 & 180 & 170 & 160 & 150 & 140 & 130 & 120 & 110 & 100 & 90 & 80 & 70 & 60 & 50 & 40 & 30 & 20 & 10 & 0\end{array}$ 

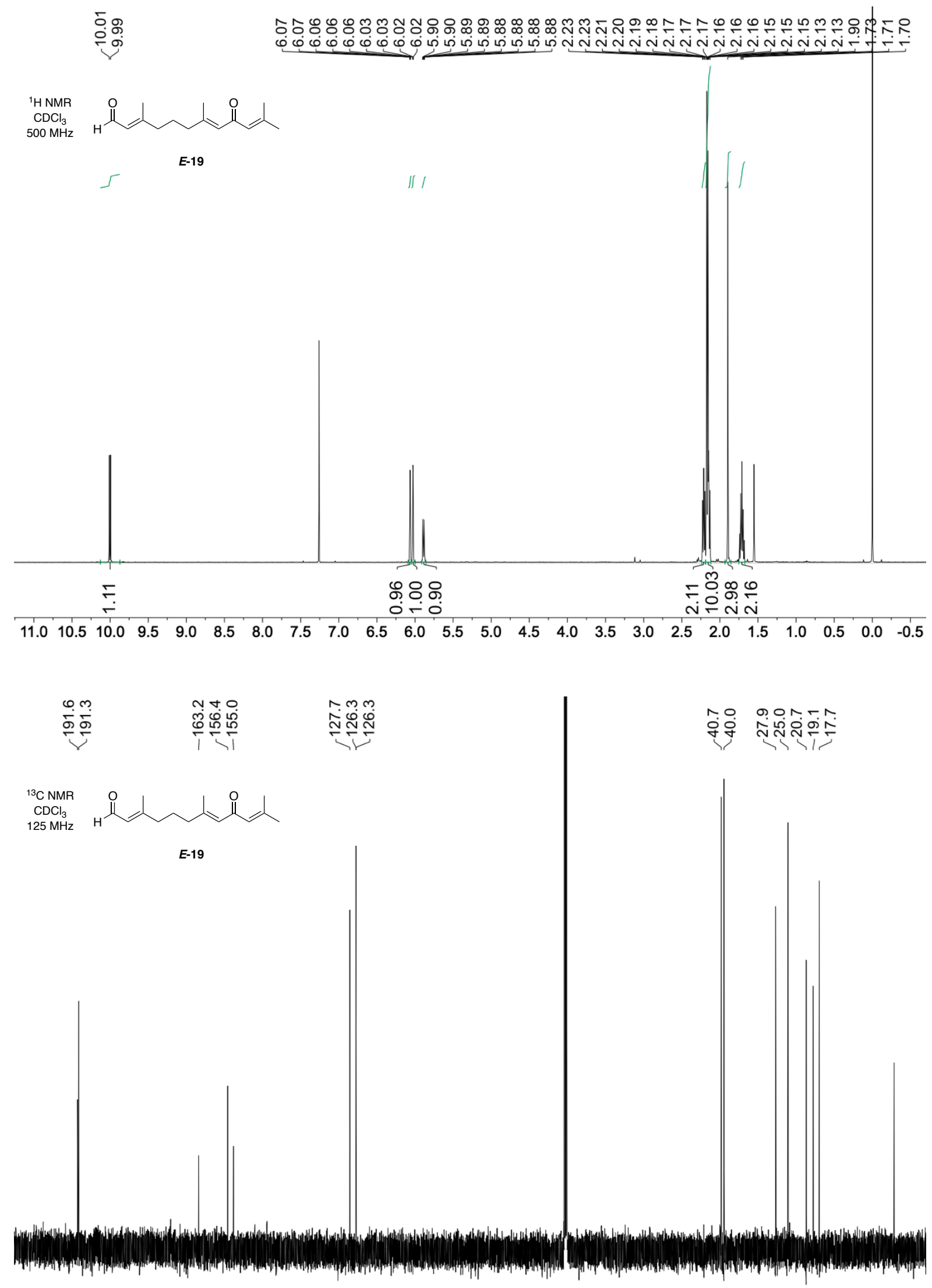

$\begin{array}{llllllllllllllllllllll}200 & 190 & 180 & 170 & 160 & 150 & 140 & 130 & 120 & 110 & 100 & 90 & 80 & 70 & 60 & 50 & 40 & 30 & 20 & 10 & 0\end{array}$ 

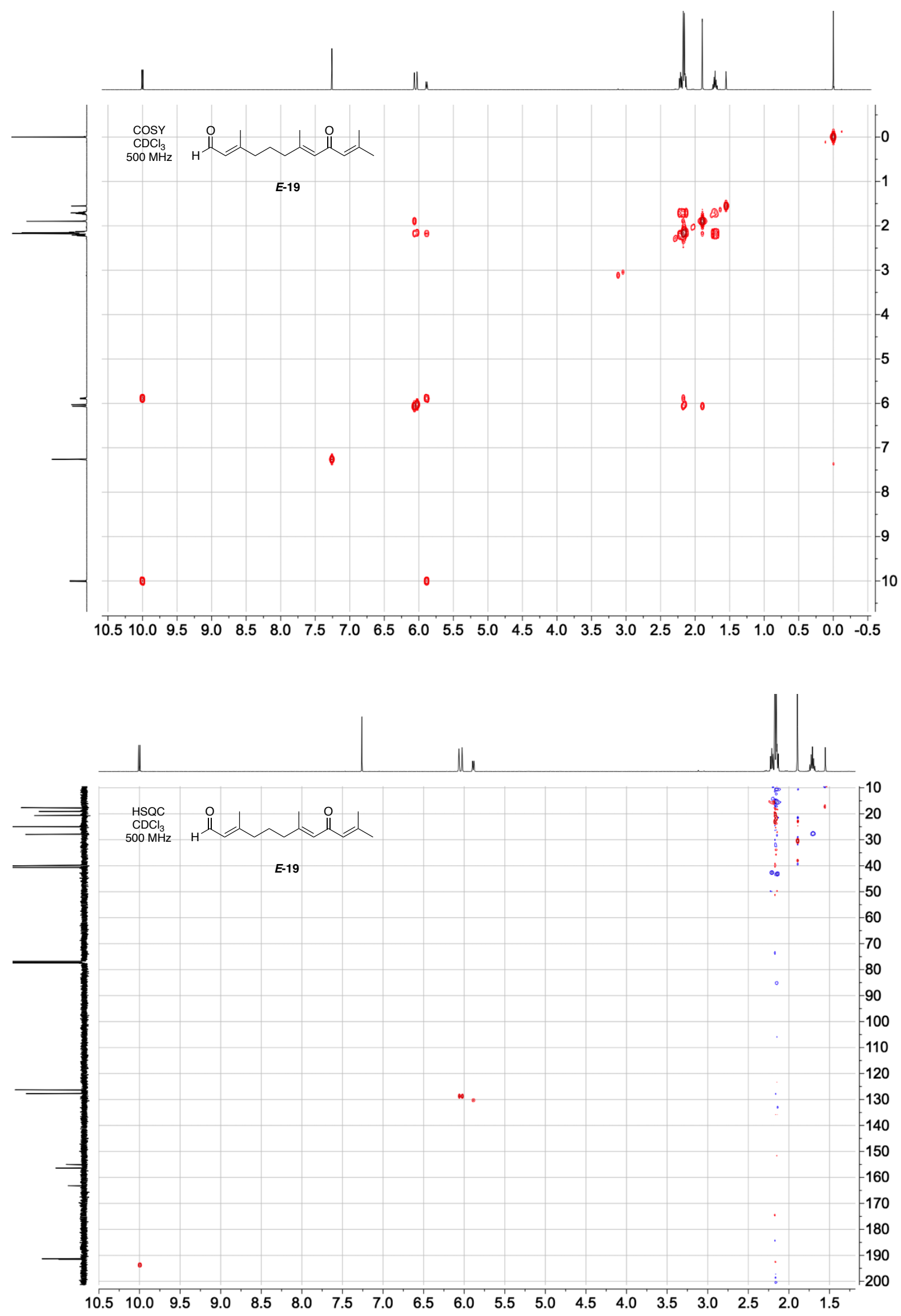

30 

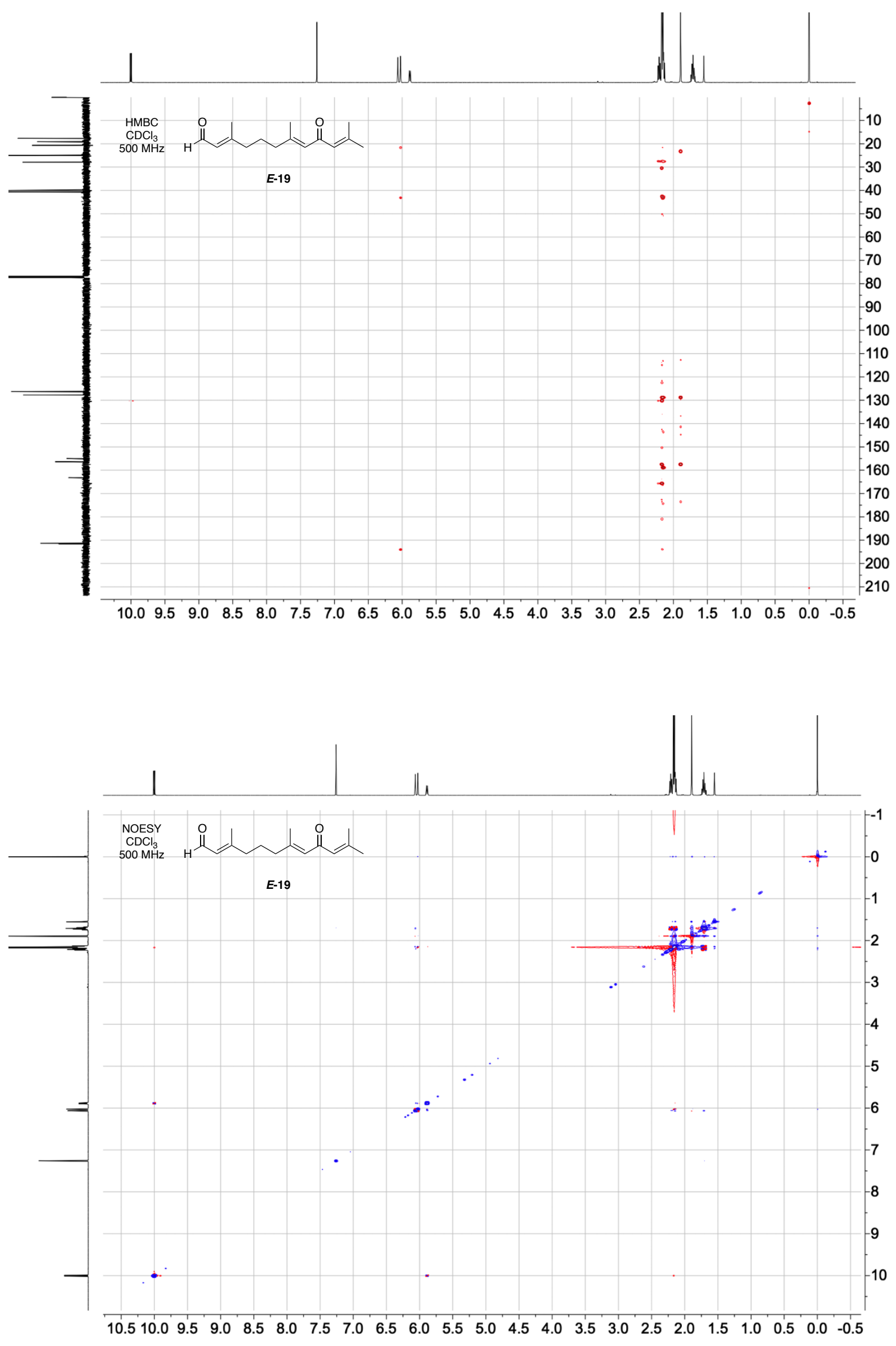

31 


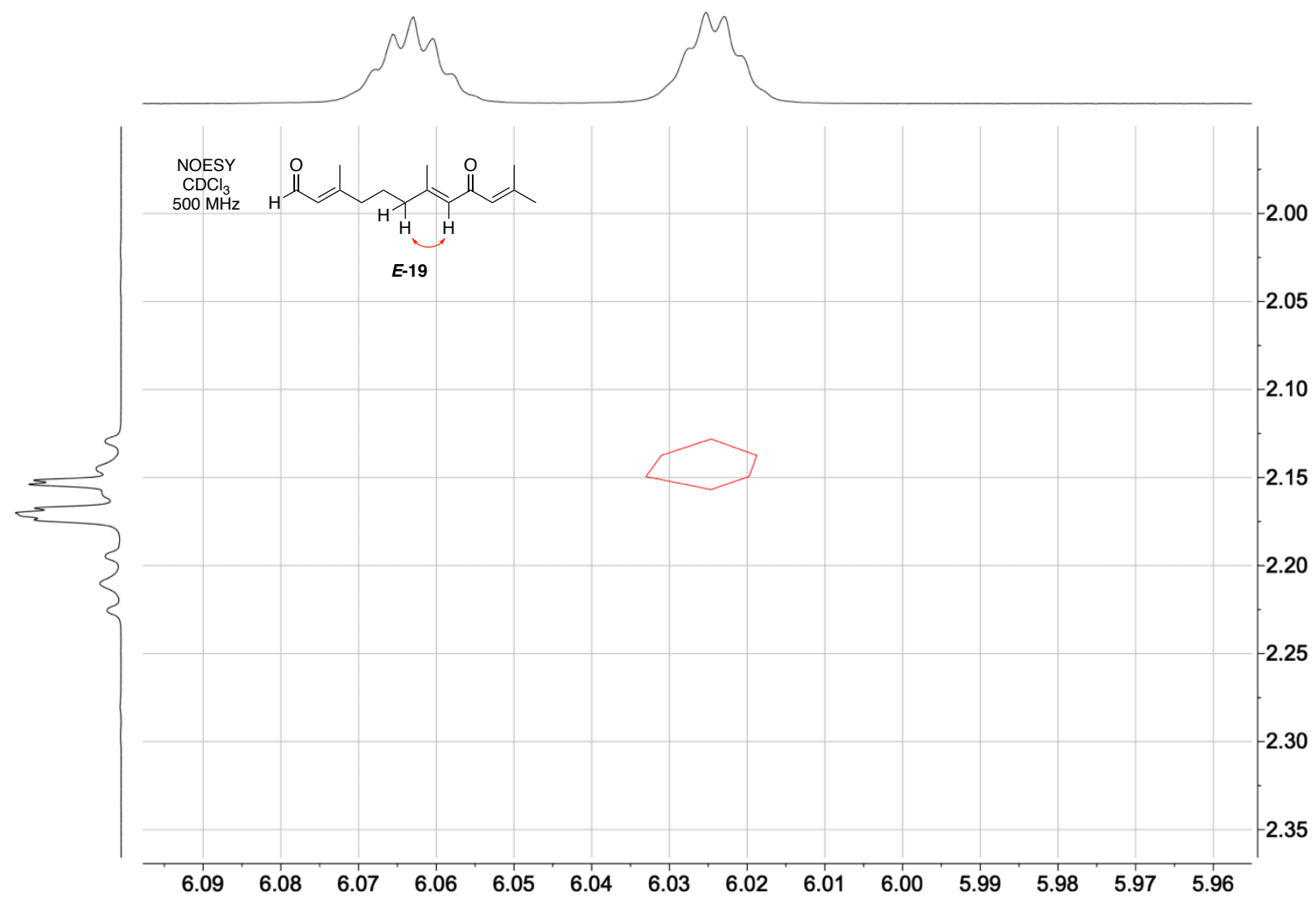



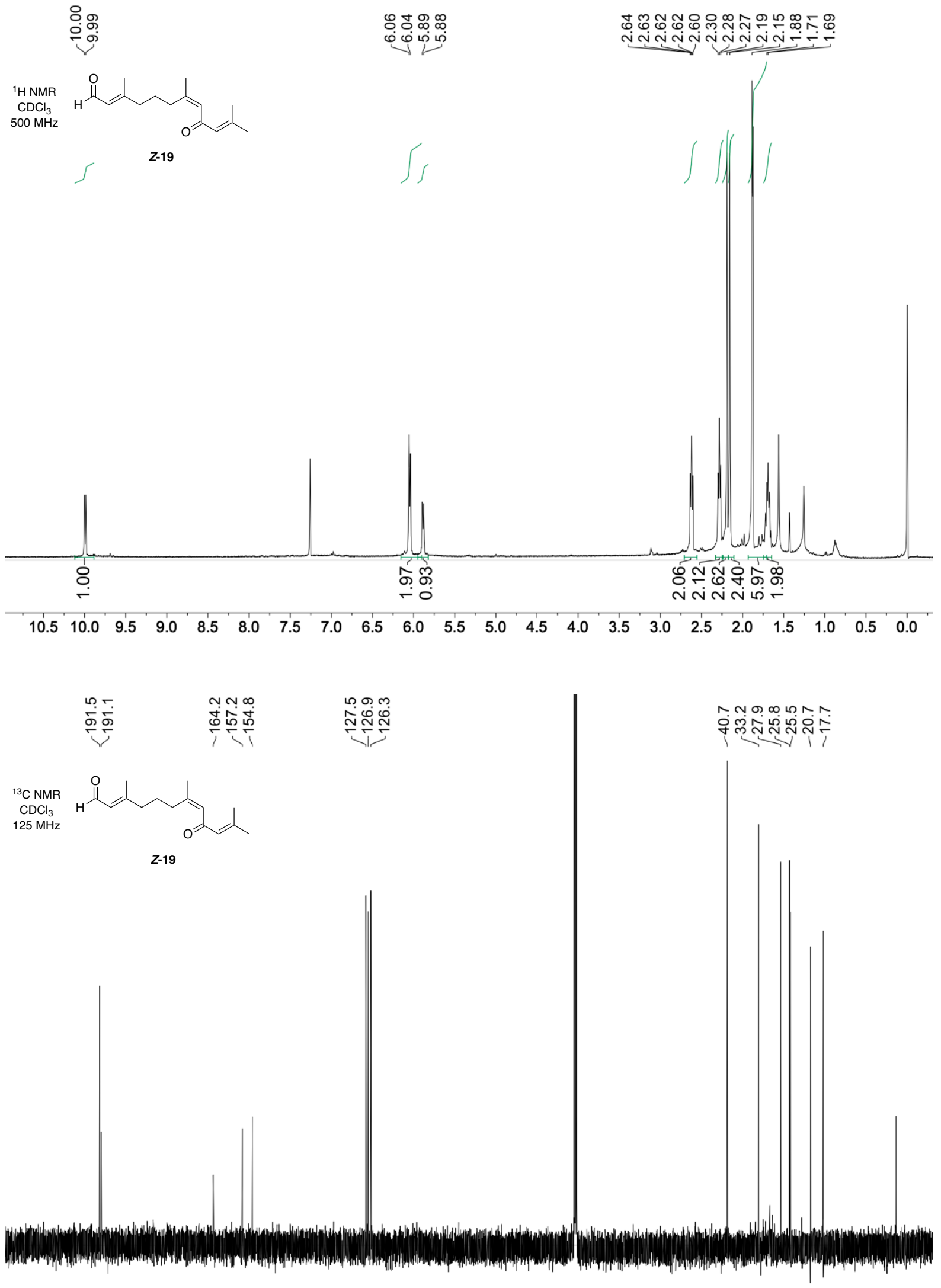

\begin{tabular}{llllllllllllllllllllll}
\hline 210 & 200 & 190 & 180 & 170 & 160 & 150 & 140 & 130 & 120 & 110 & 100 & 90 & 80 & 70 & 60 & 50 & 40 & 30 & 20 & 10 & 0
\end{tabular} 

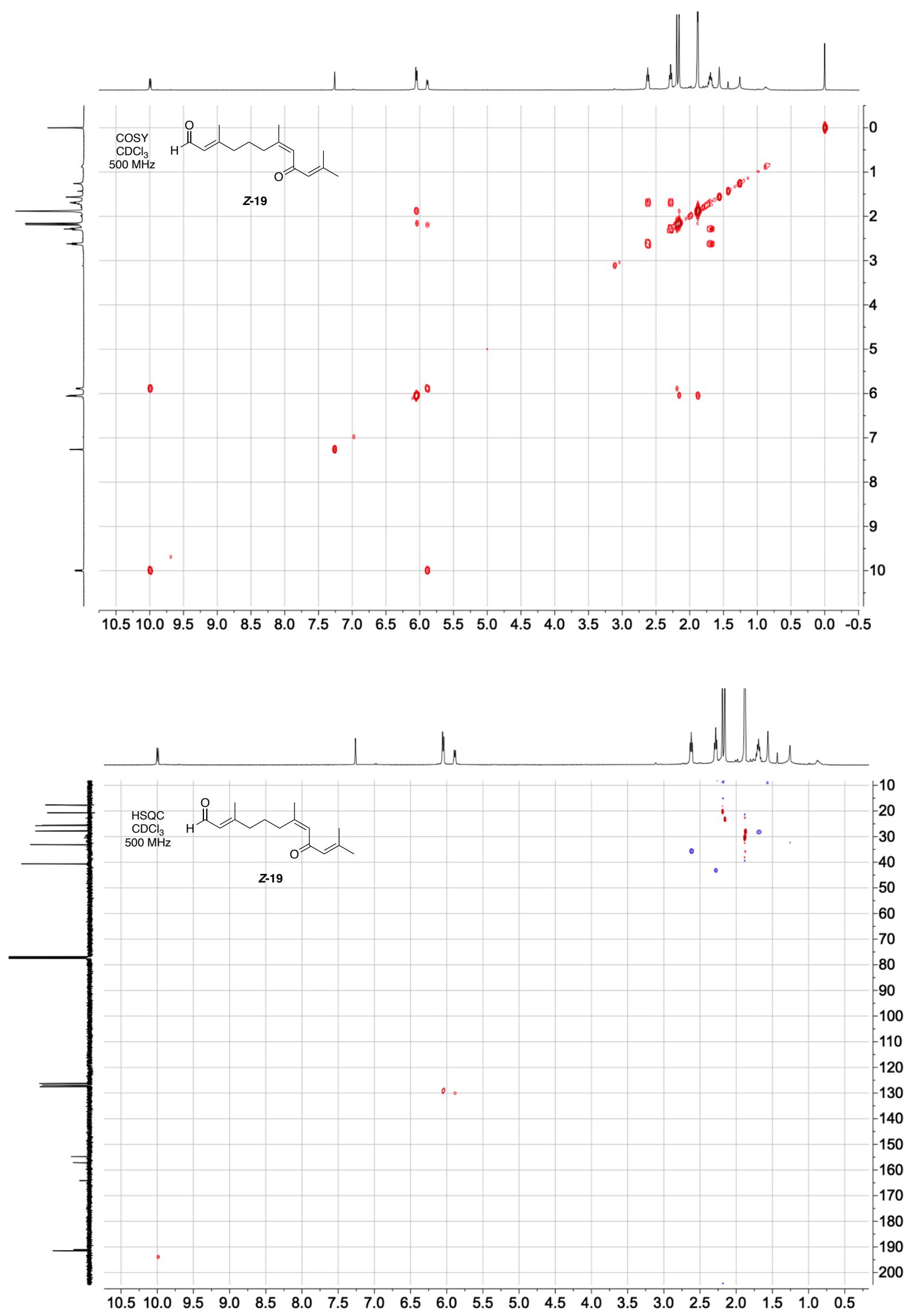

34 

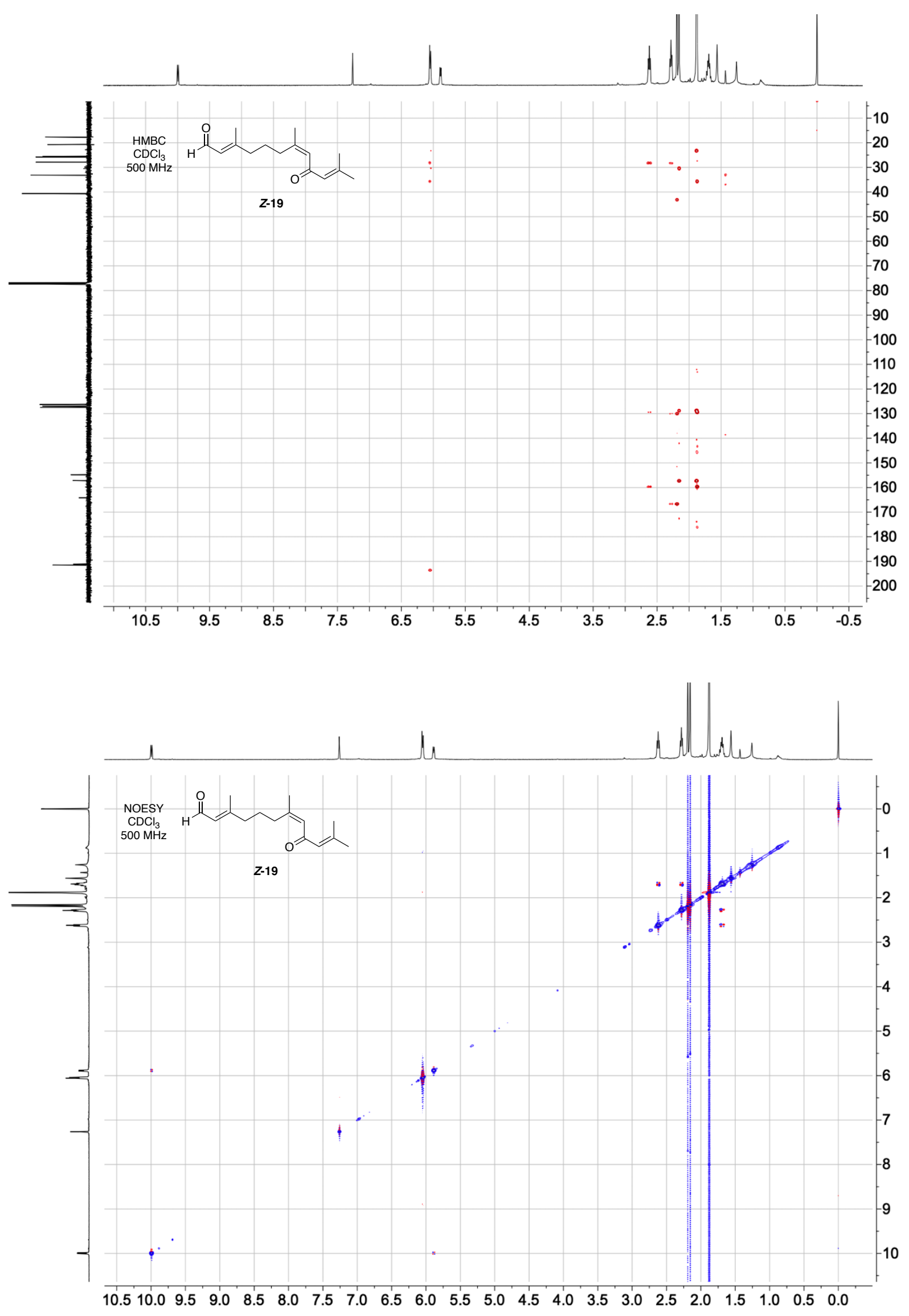

35 

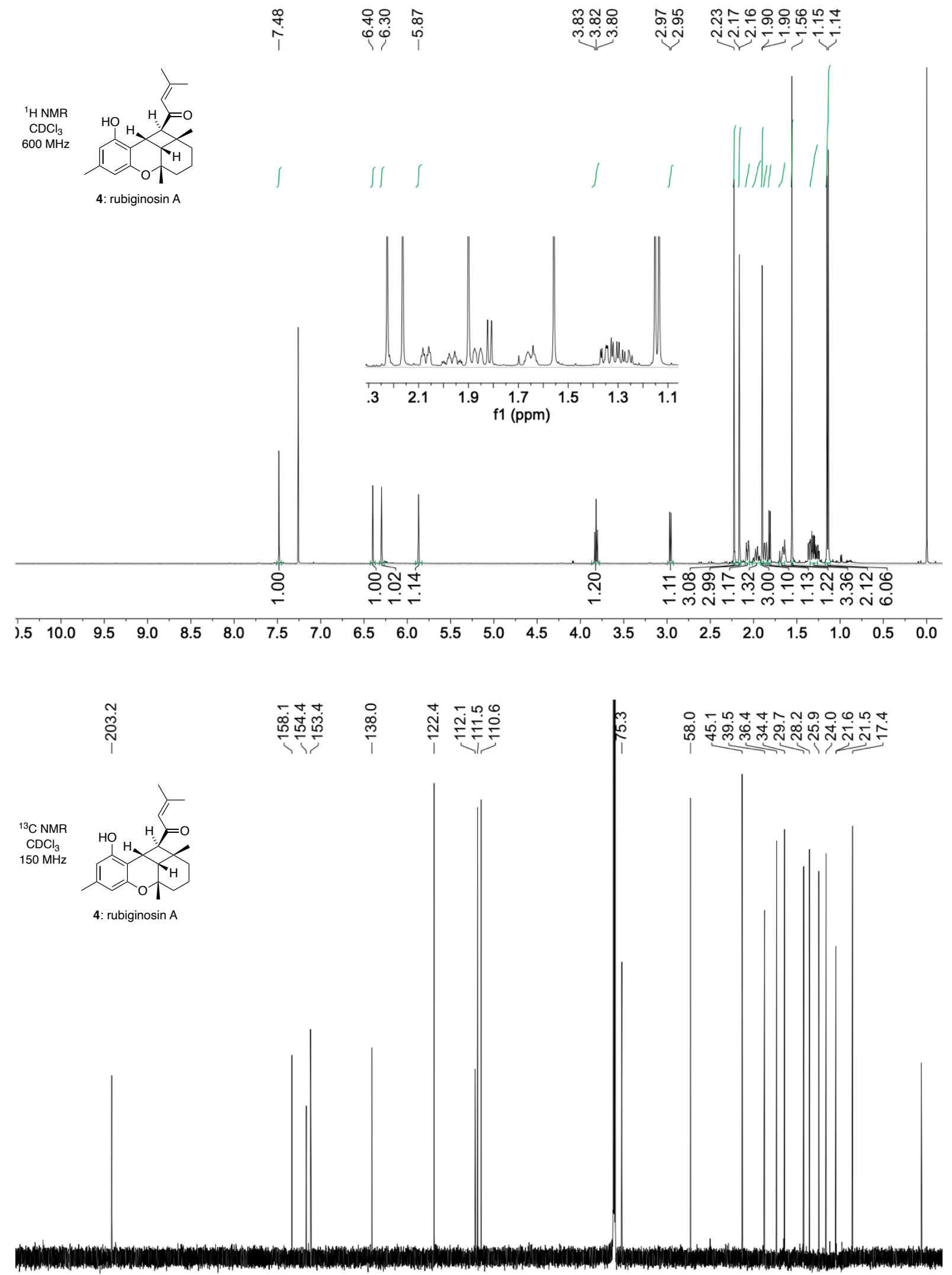

$\begin{array}{llllllllllllllllllllllllll}220 & 210 & 200 & 190 & 180 & 170 & 160 & 150 & 140 & 130 & 120 & 110 & 100 & 90 & 80 & 70 & 60 & 50 & 40 & 30 & 20 & 10 & 0\end{array}$ 

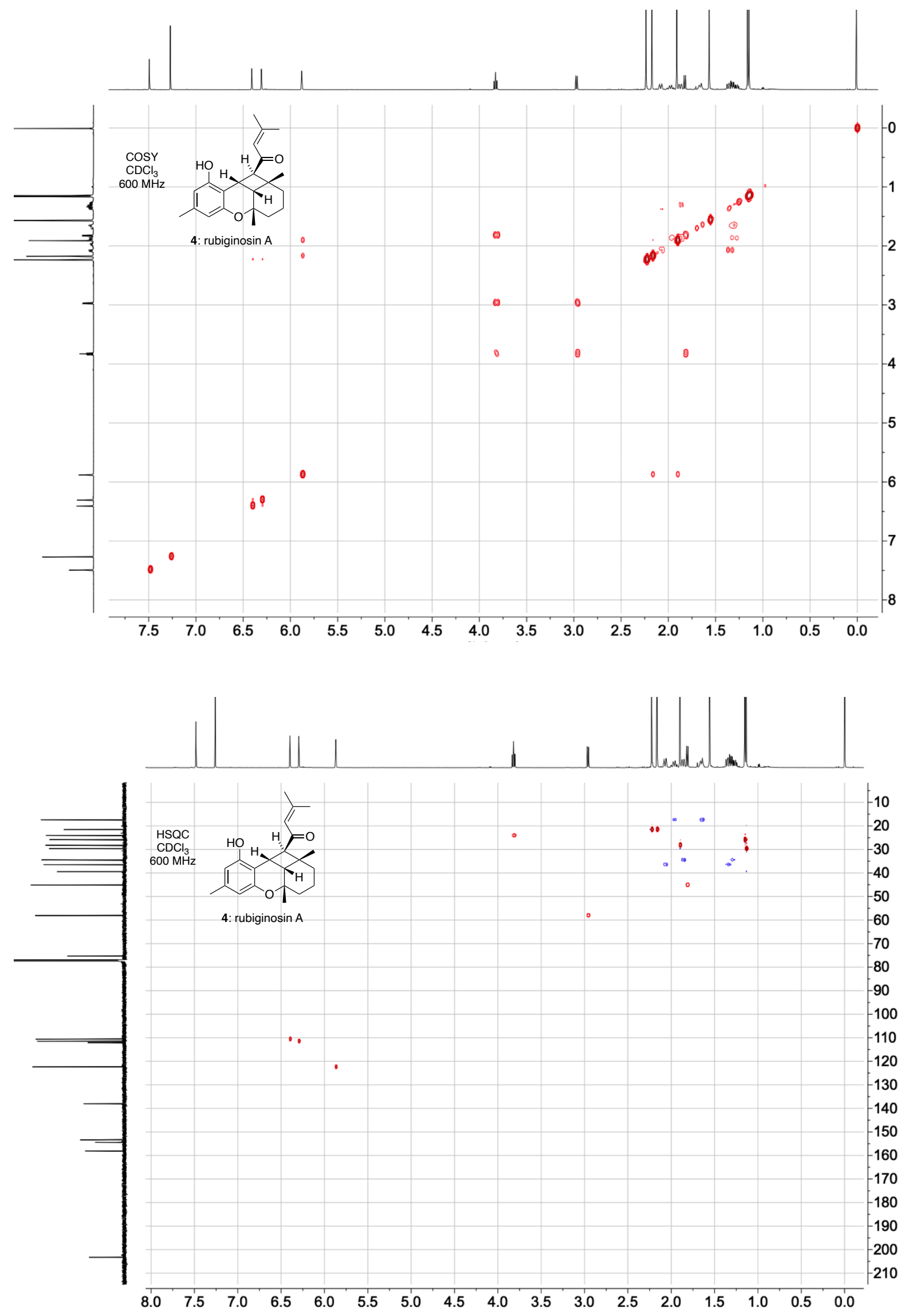

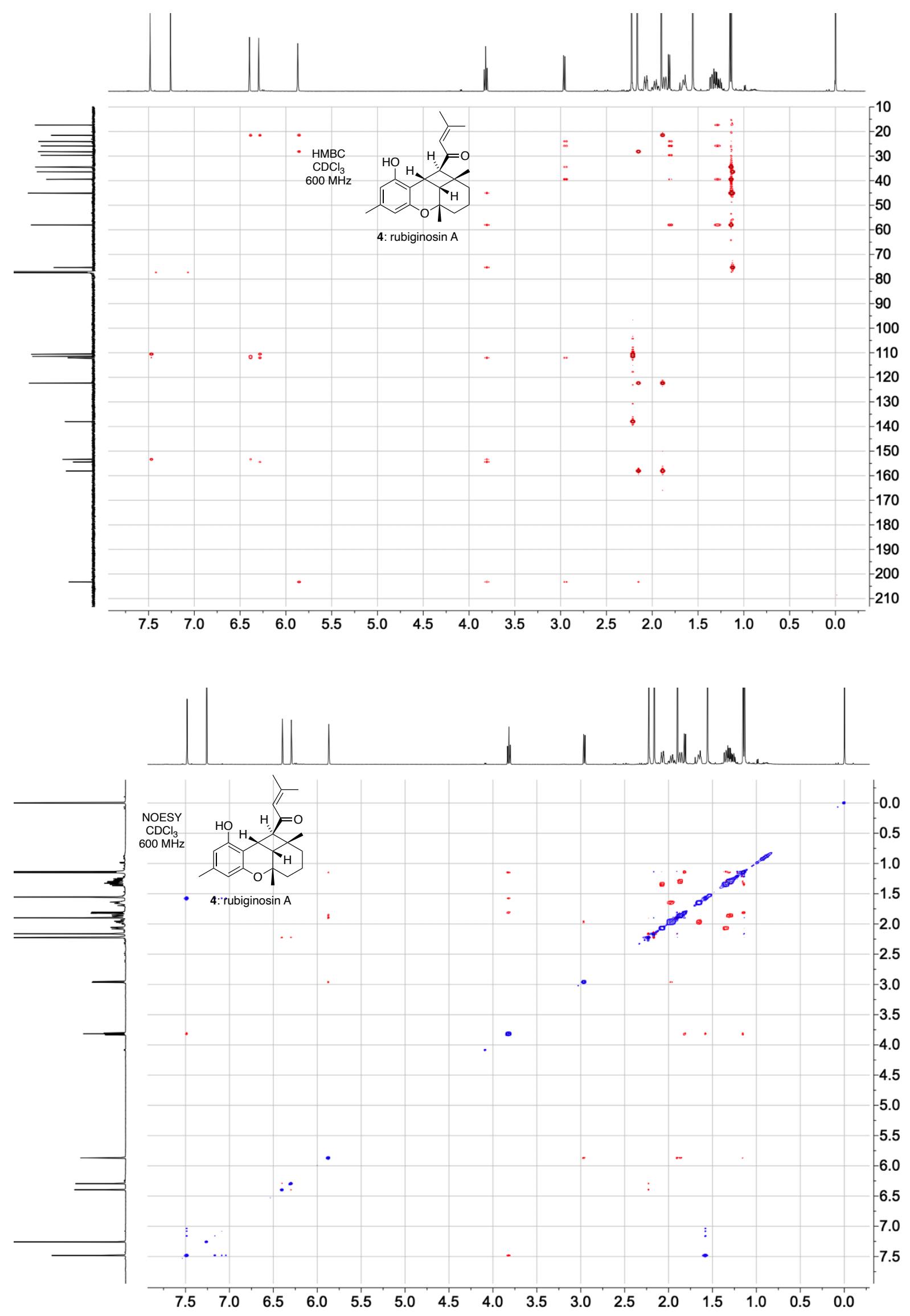

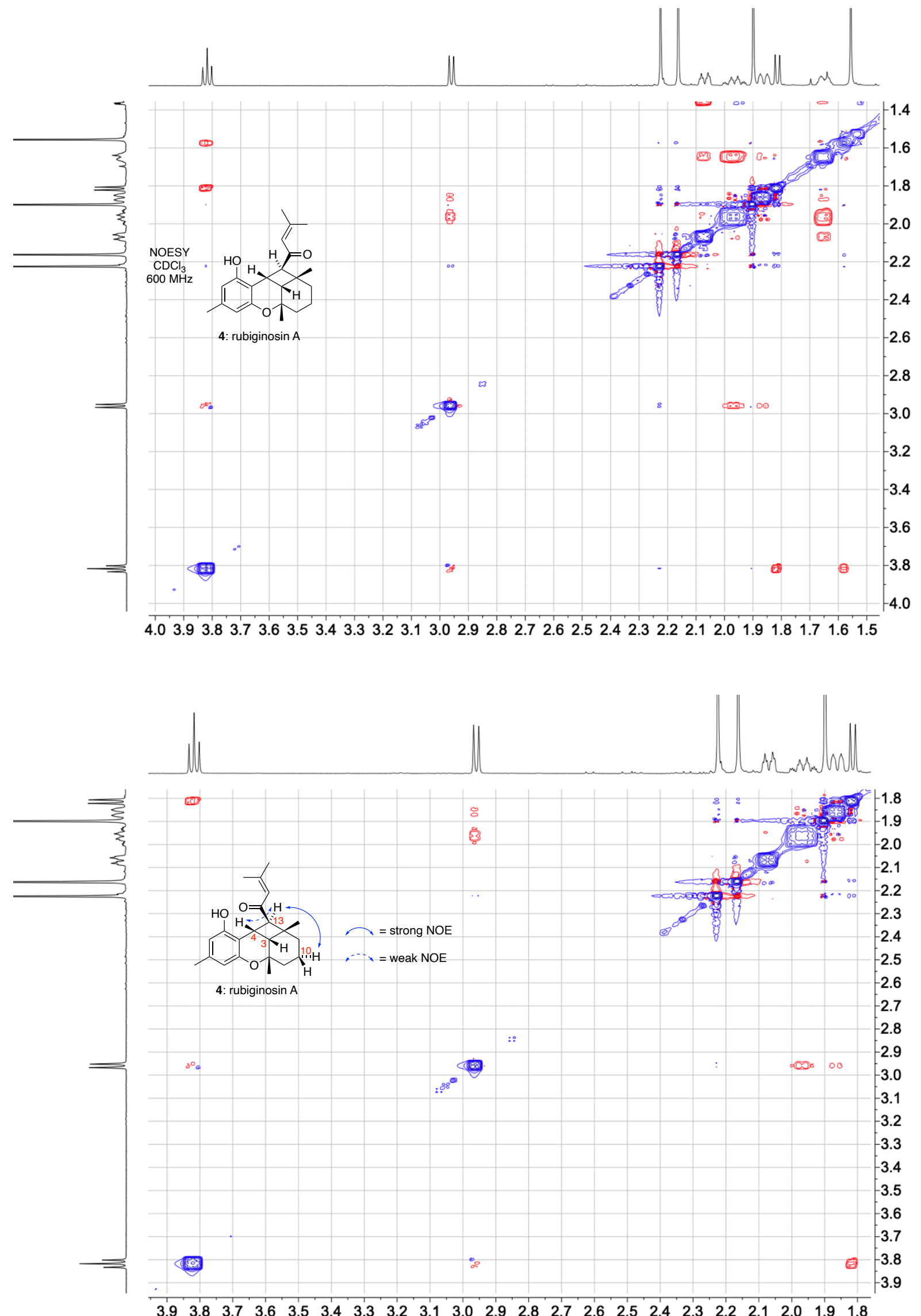

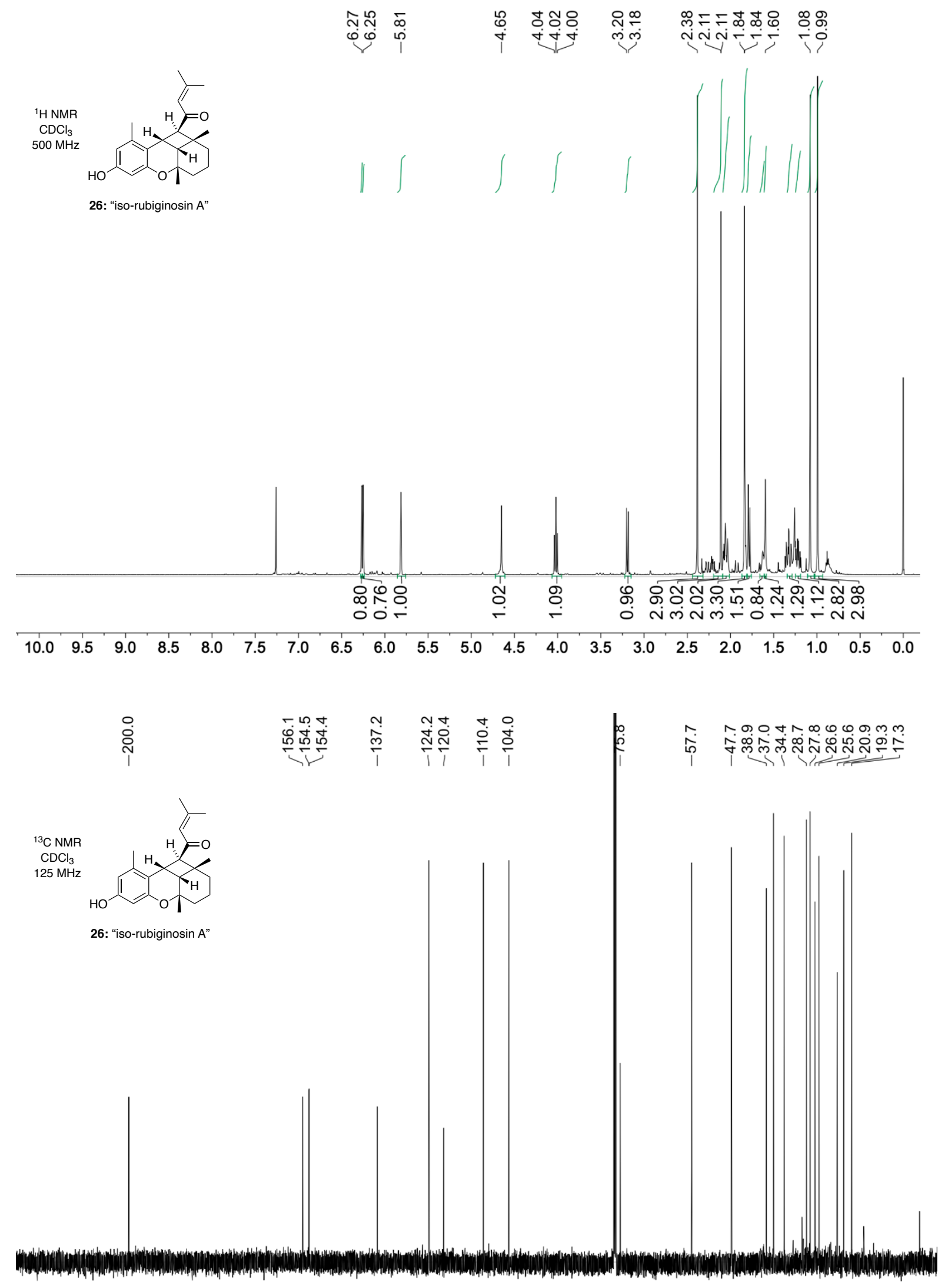

$\begin{array}{lllllllllllllllllllllllll}220 & 210 & 200 & 190 & 180 & 170 & 160 & 150 & 140 & 130 & 120 & 110 & 100 & 90 & 80 & 70 & 60 & 50 & 40 & 30 & 20 & 10 & 0\end{array}$ 

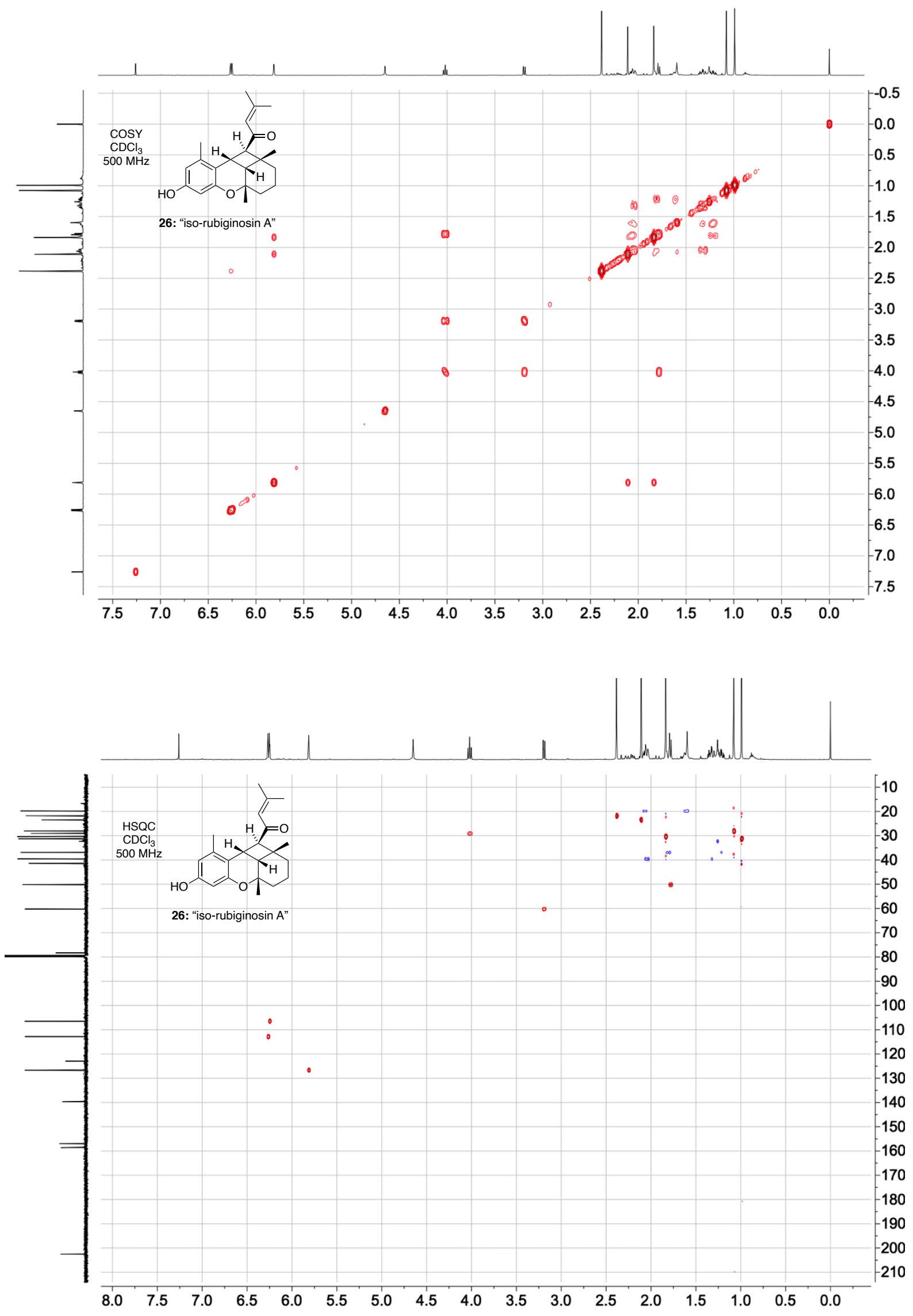

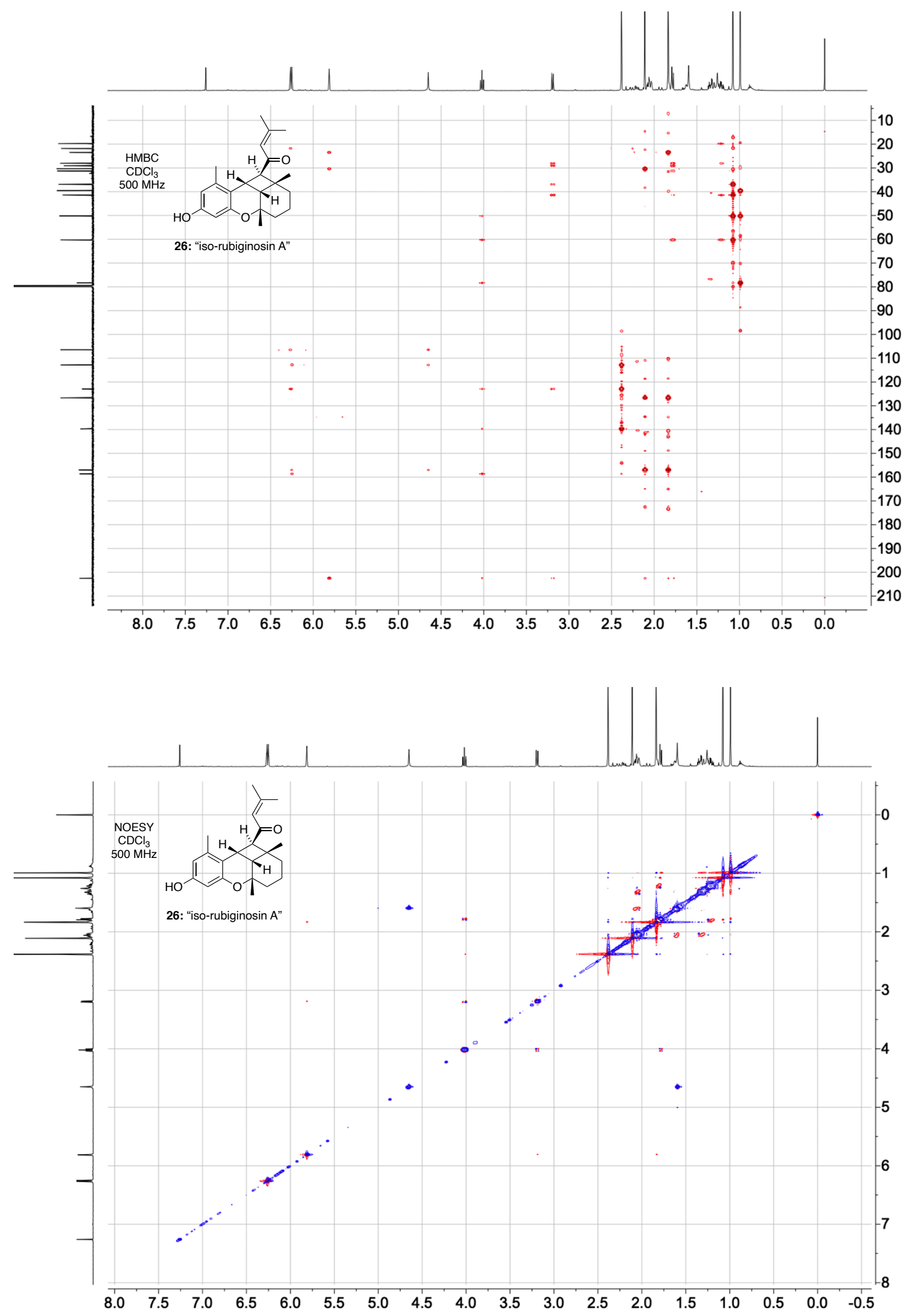


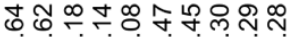

0ं

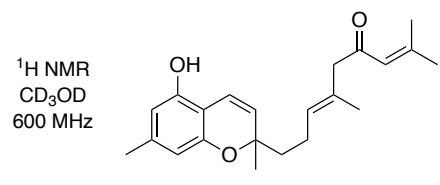

11: rubiginosin $E$

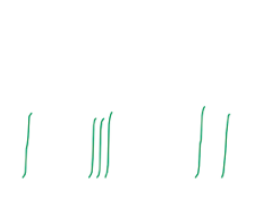

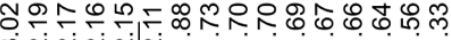

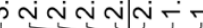

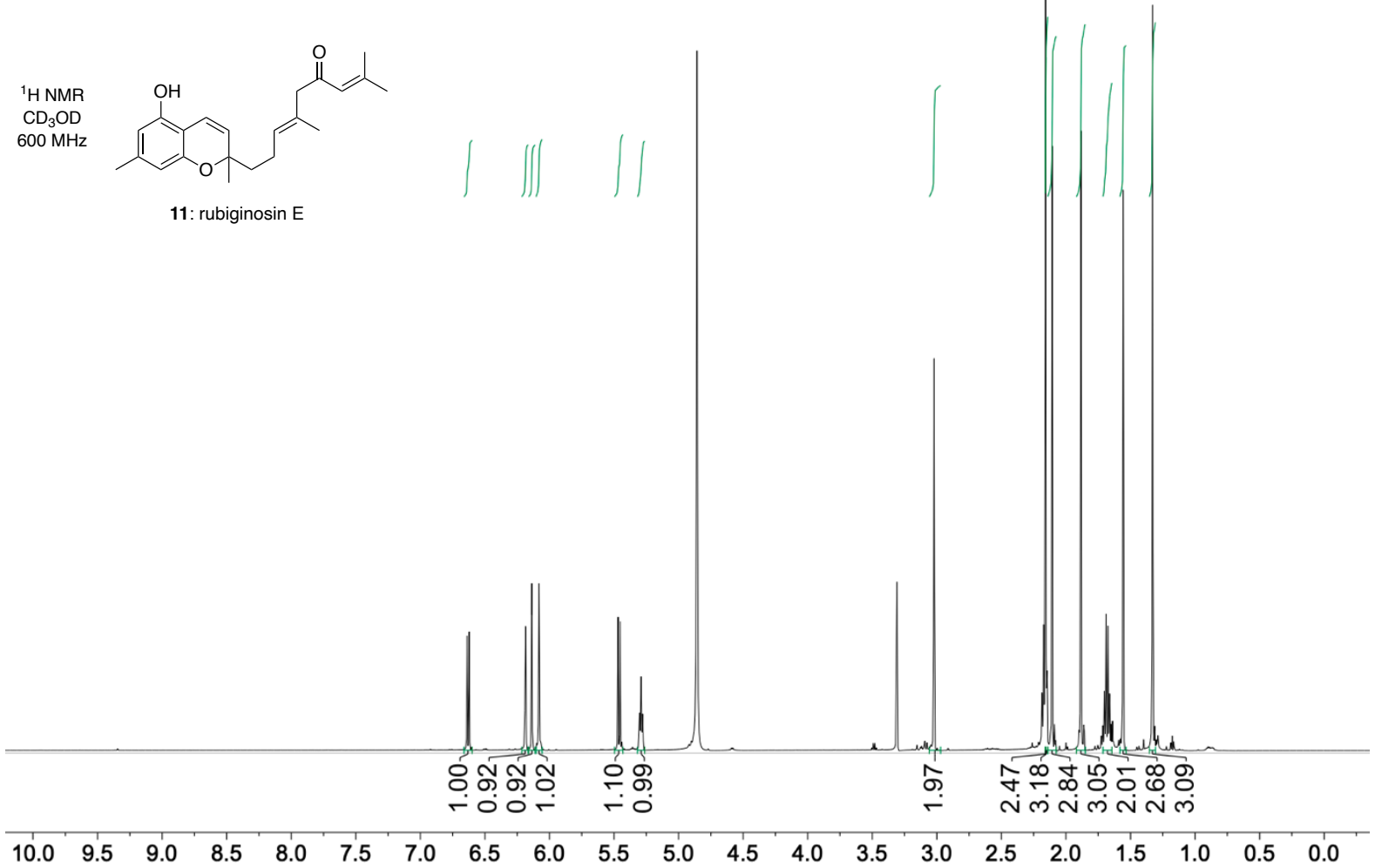

ঙำ

方少

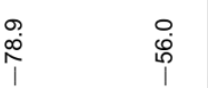

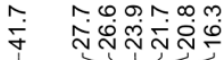

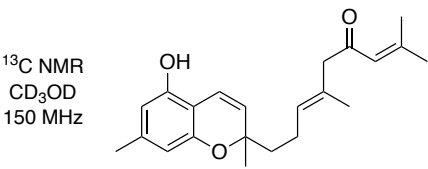

11: rubiginosin $E$

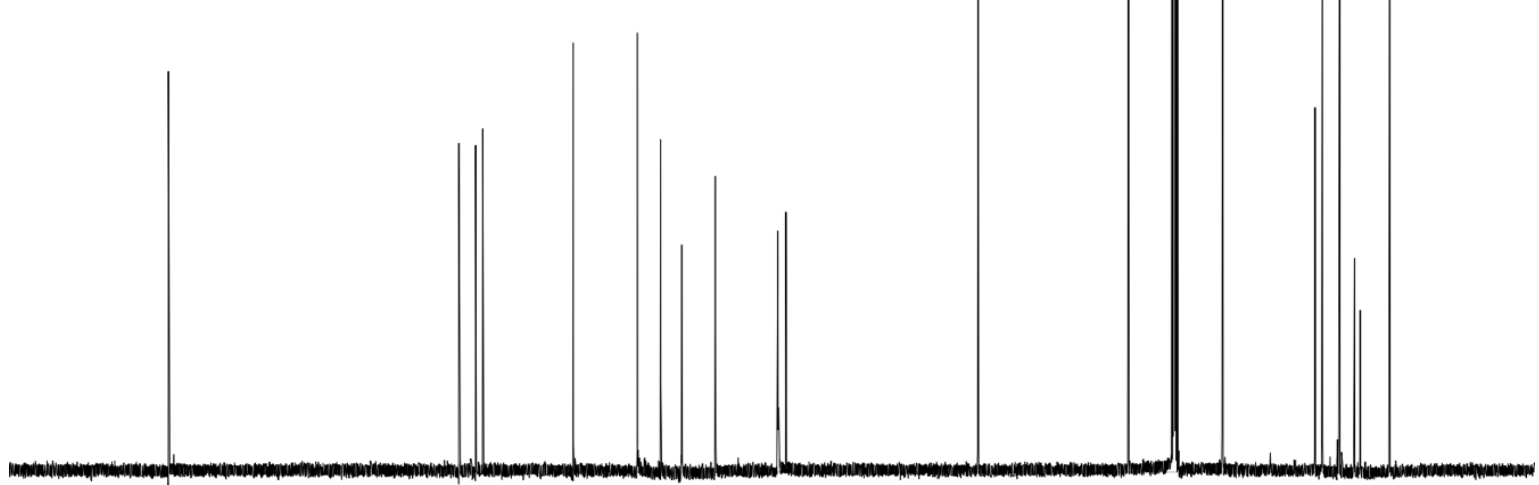

$\begin{array}{lllllllllllllllllllllllll}220 & 210 & 200 & 190 & 180 & 170 & 160 & 150 & 140 & 130 & 120 & 110 & 100 & 90 & 80 & 70 & 60 & 50 & 40 & 30 & 20 & 10 & 0\end{array}$ 

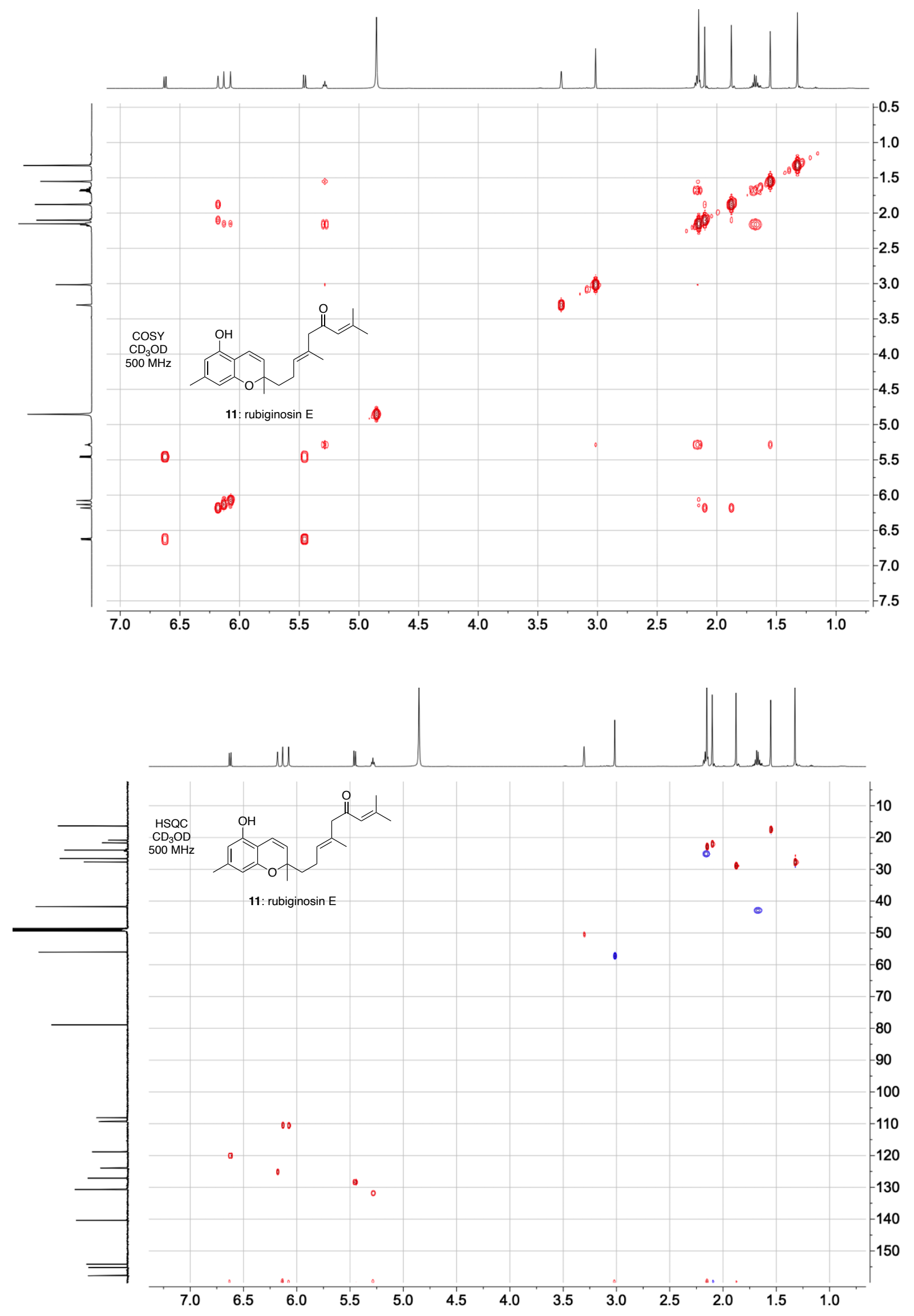


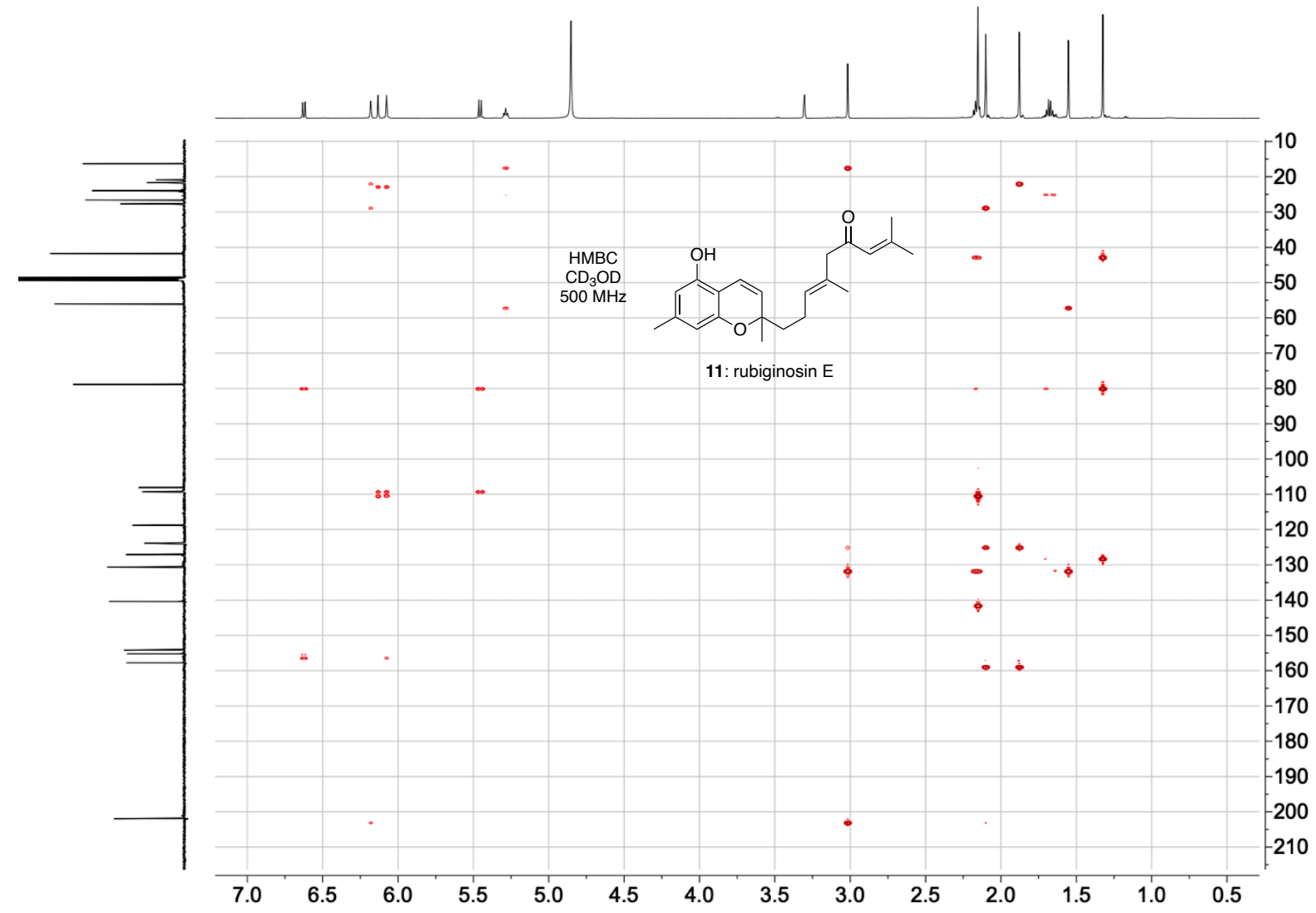



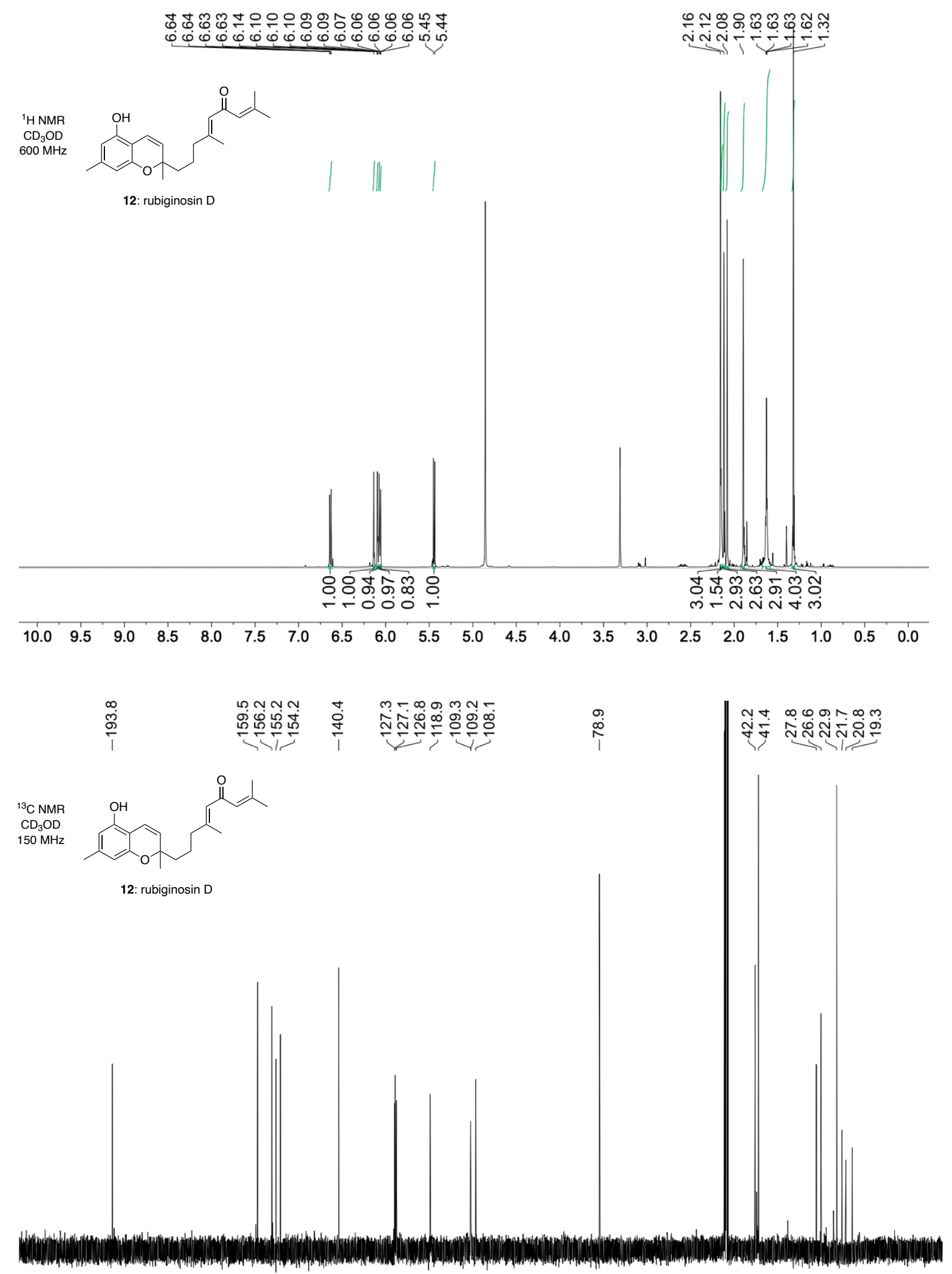

$\begin{array}{lllllllllllllllllllllllllll}210 & 200 & 190 & 180 & 170 & 160 & 150 & 140 & 130 & 120 & 110 & 100 & 90 & 80 & 70 & 60 & 50 & 40 & 30 & 20 & 10 & 0\end{array}$ 

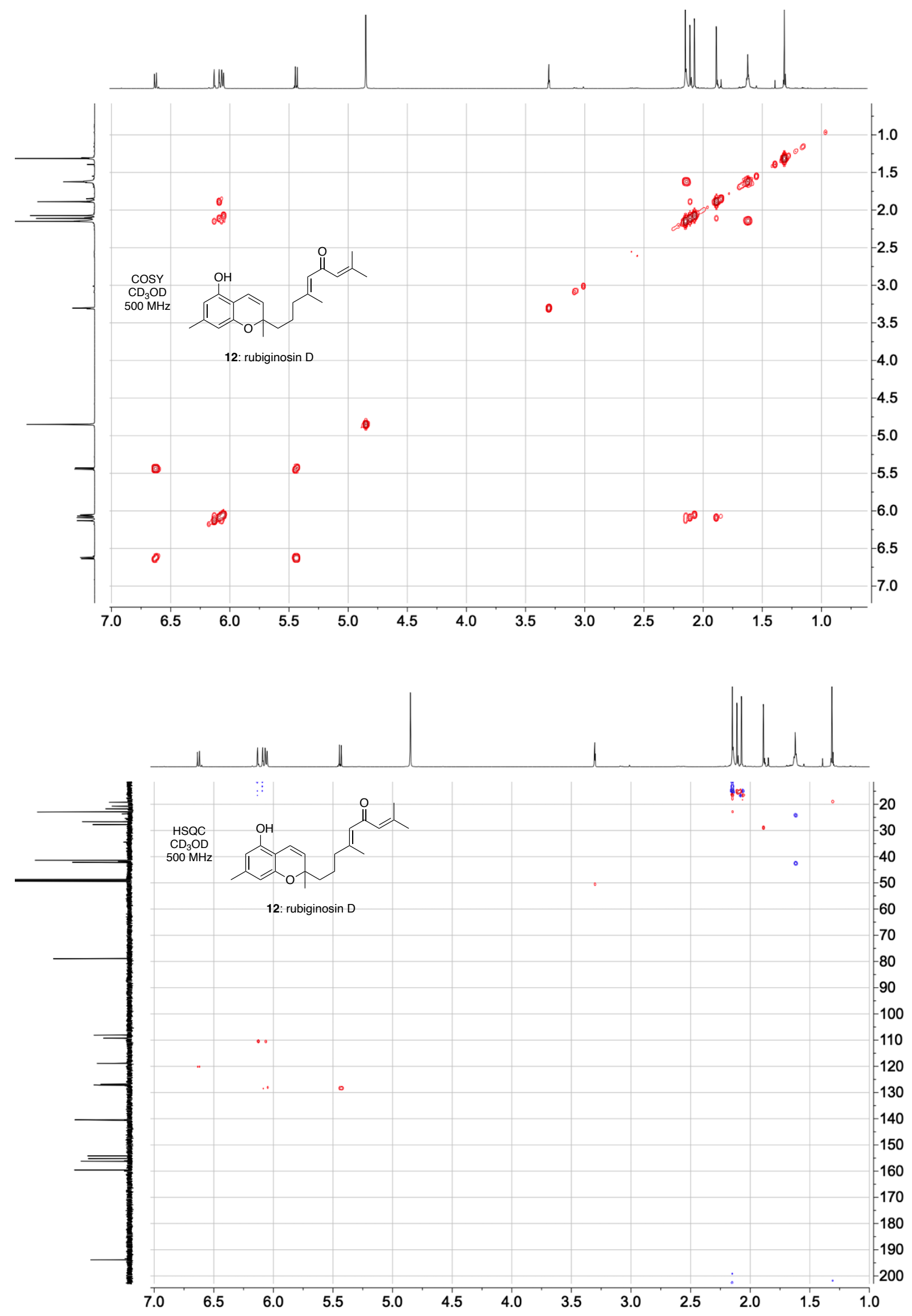


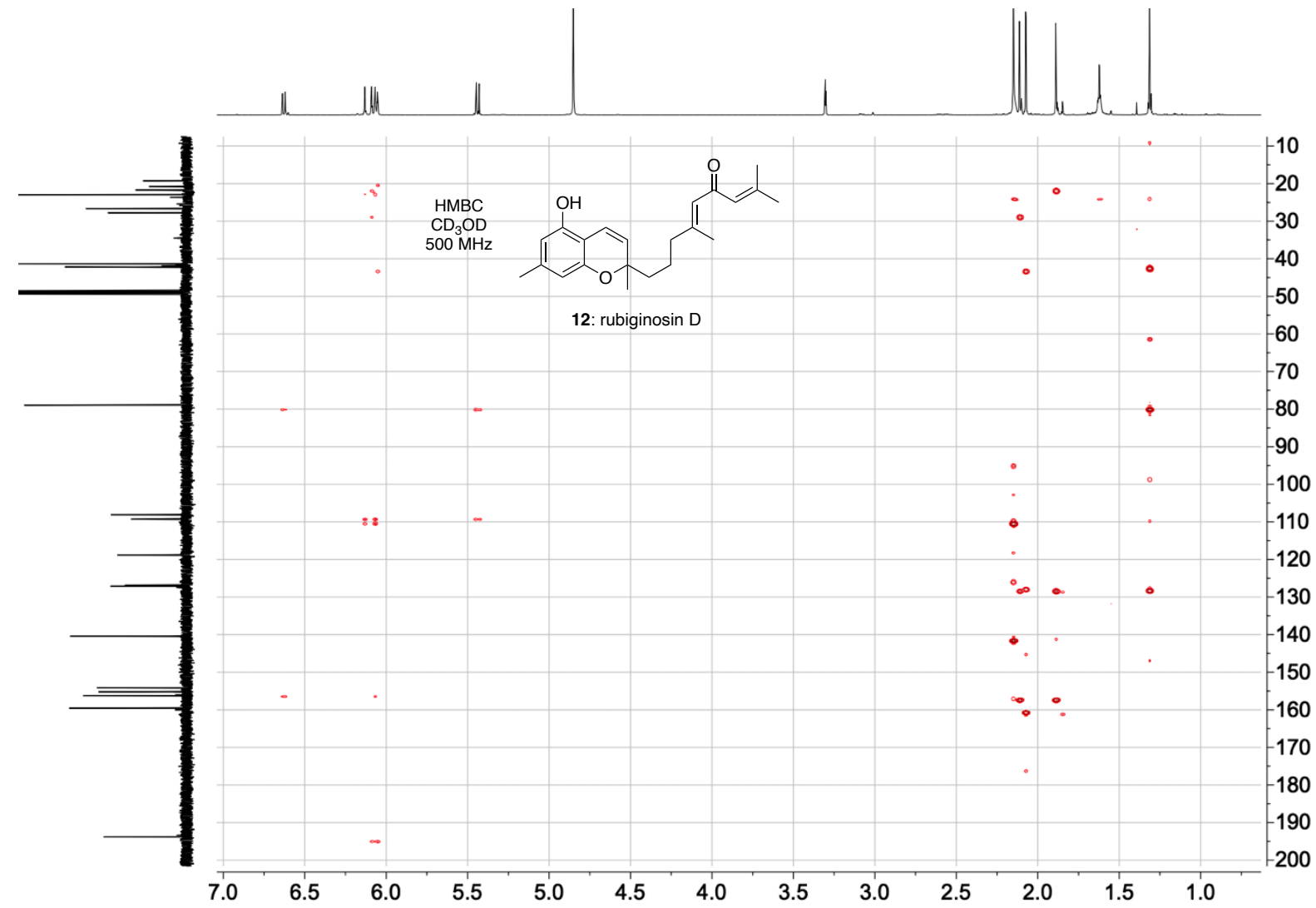



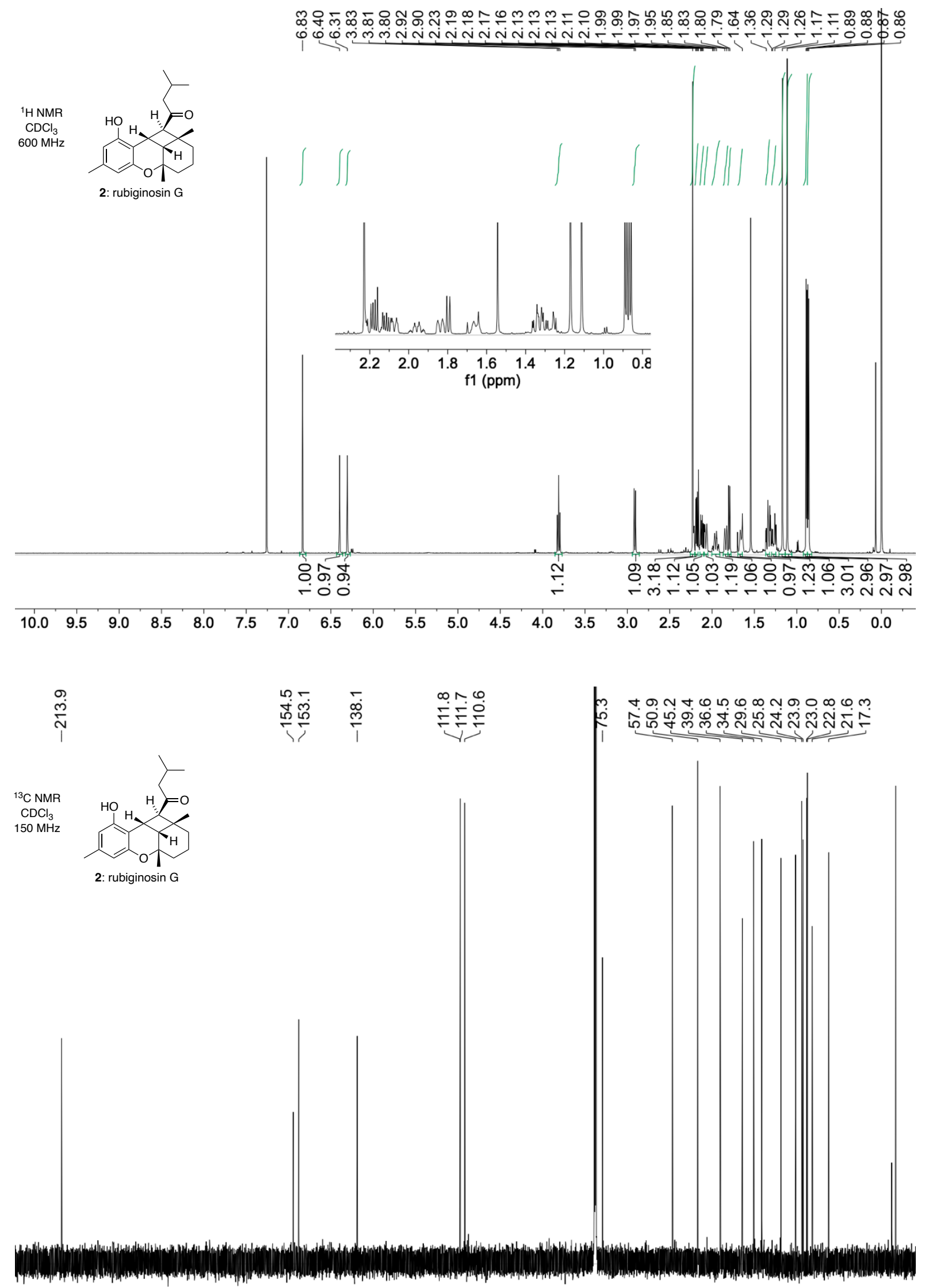

$\begin{array}{llllllllllllllllllllllll}220 & 210 & 200 & 190 & 180 & 170 & 160 & 150 & 140 & 130 & 120 & 110 & 100 & 90 & 80 & 70 & 60 & 50 & 40 & 30 & 20 & 10 & 0\end{array}$ 

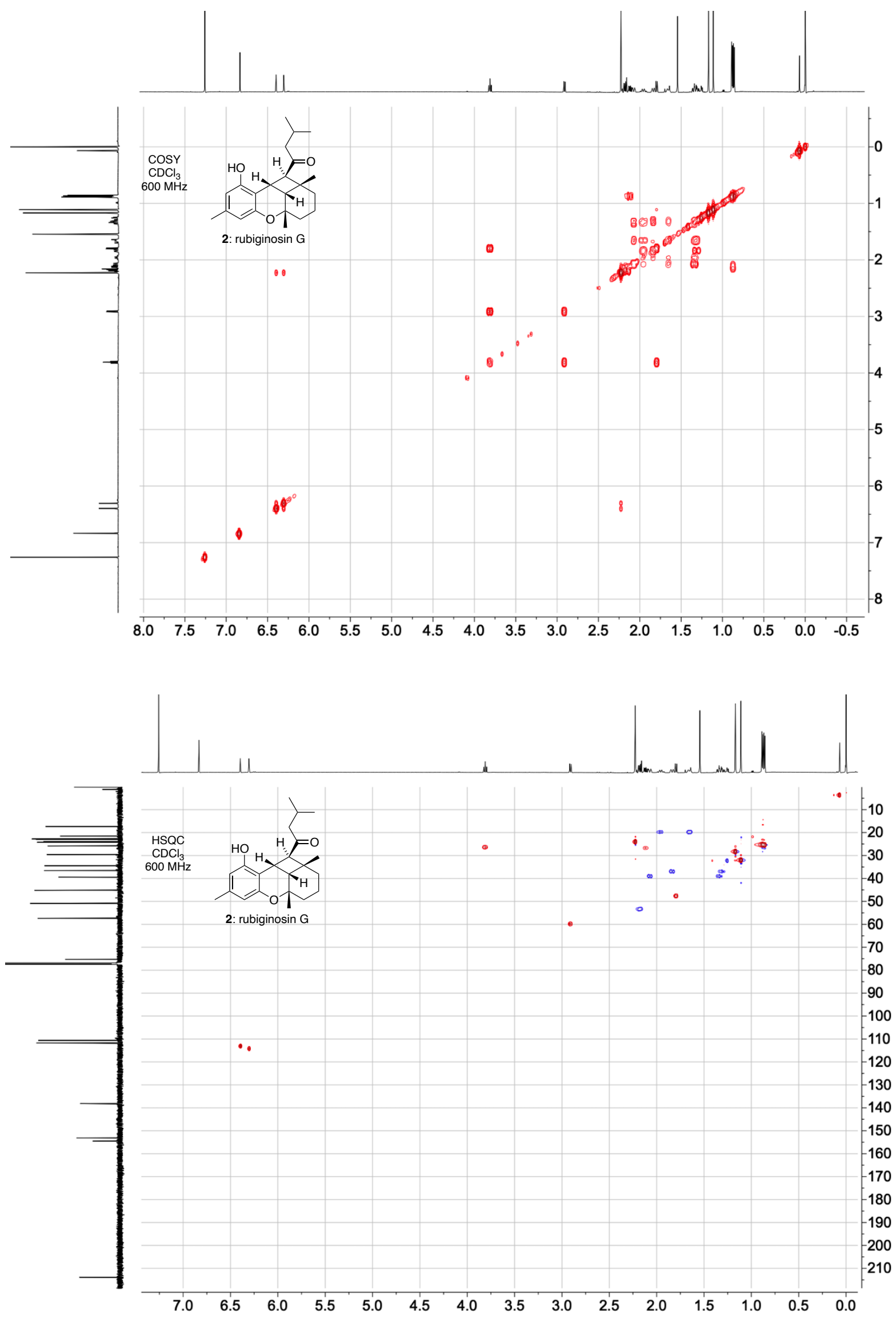

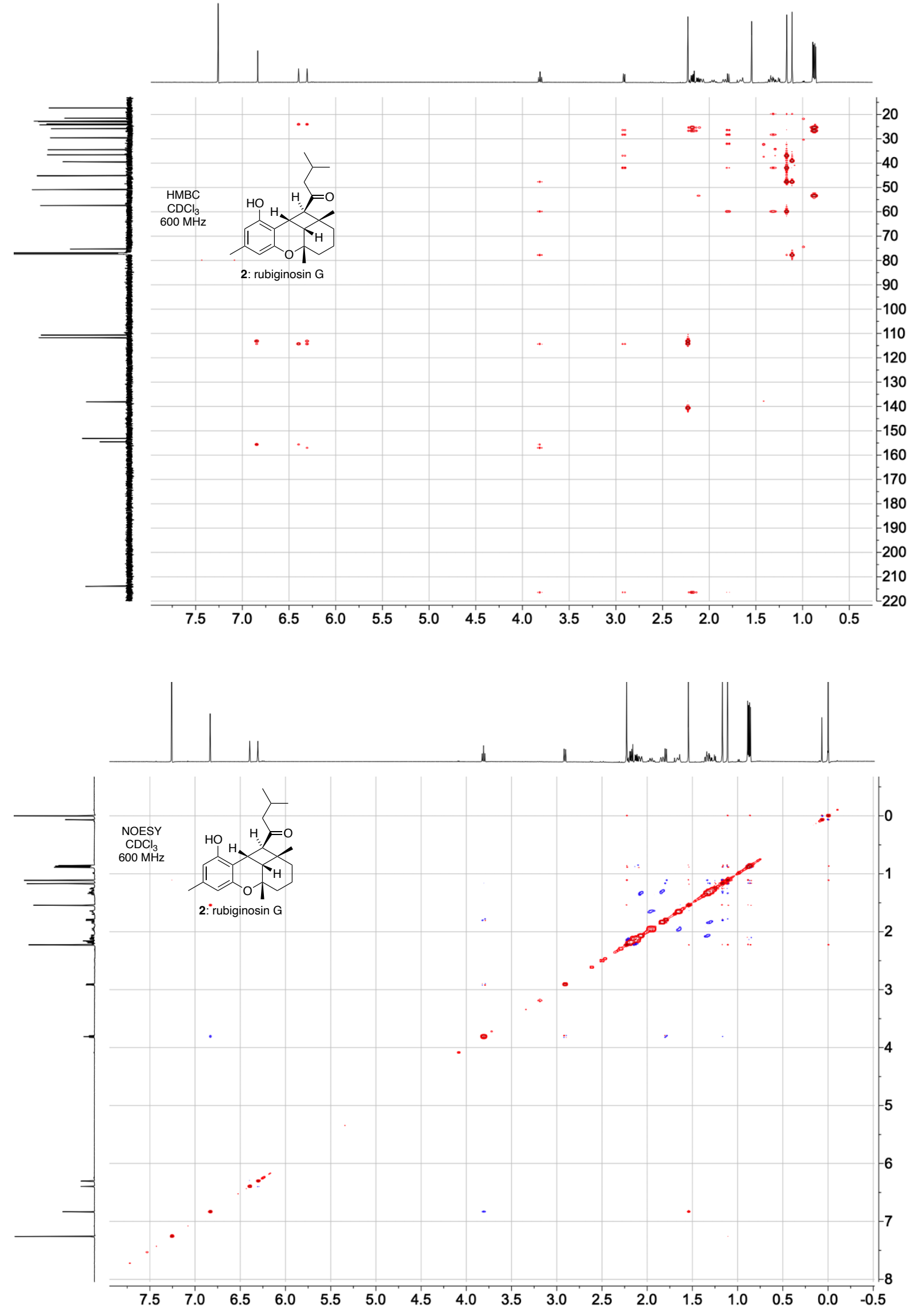

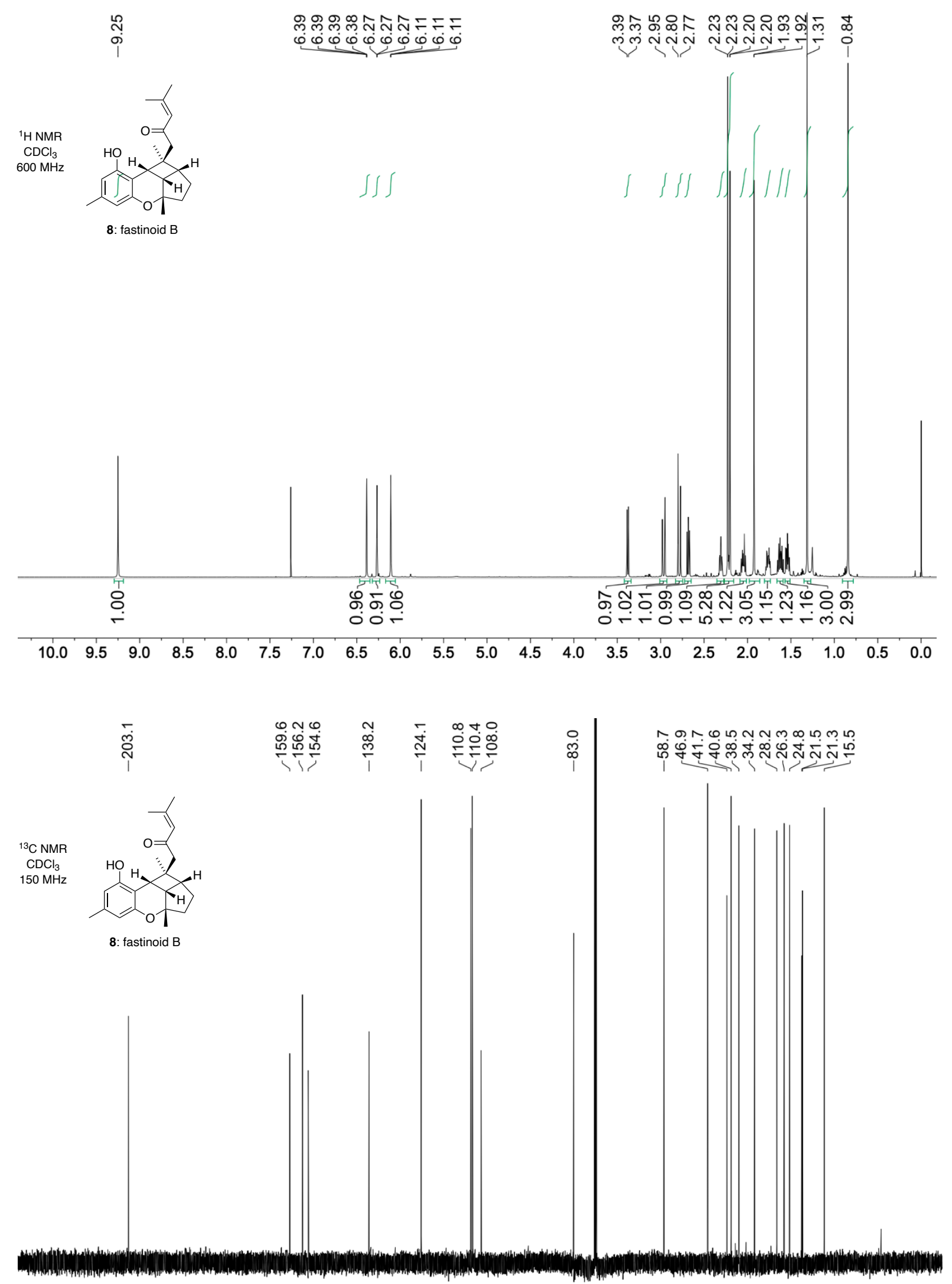

\begin{tabular}{llllllllllllllllllllllllllllll}
\hline 230 & 220 & 210 & 200 & 190 & 180 & 170 & 160 & 150 & 140 & 130 & 120 & 110 & 100 & 90 & 80 & 70 & 60 & 50 & 40 & 30 & 20 & 10 & 0 & -10
\end{tabular} 

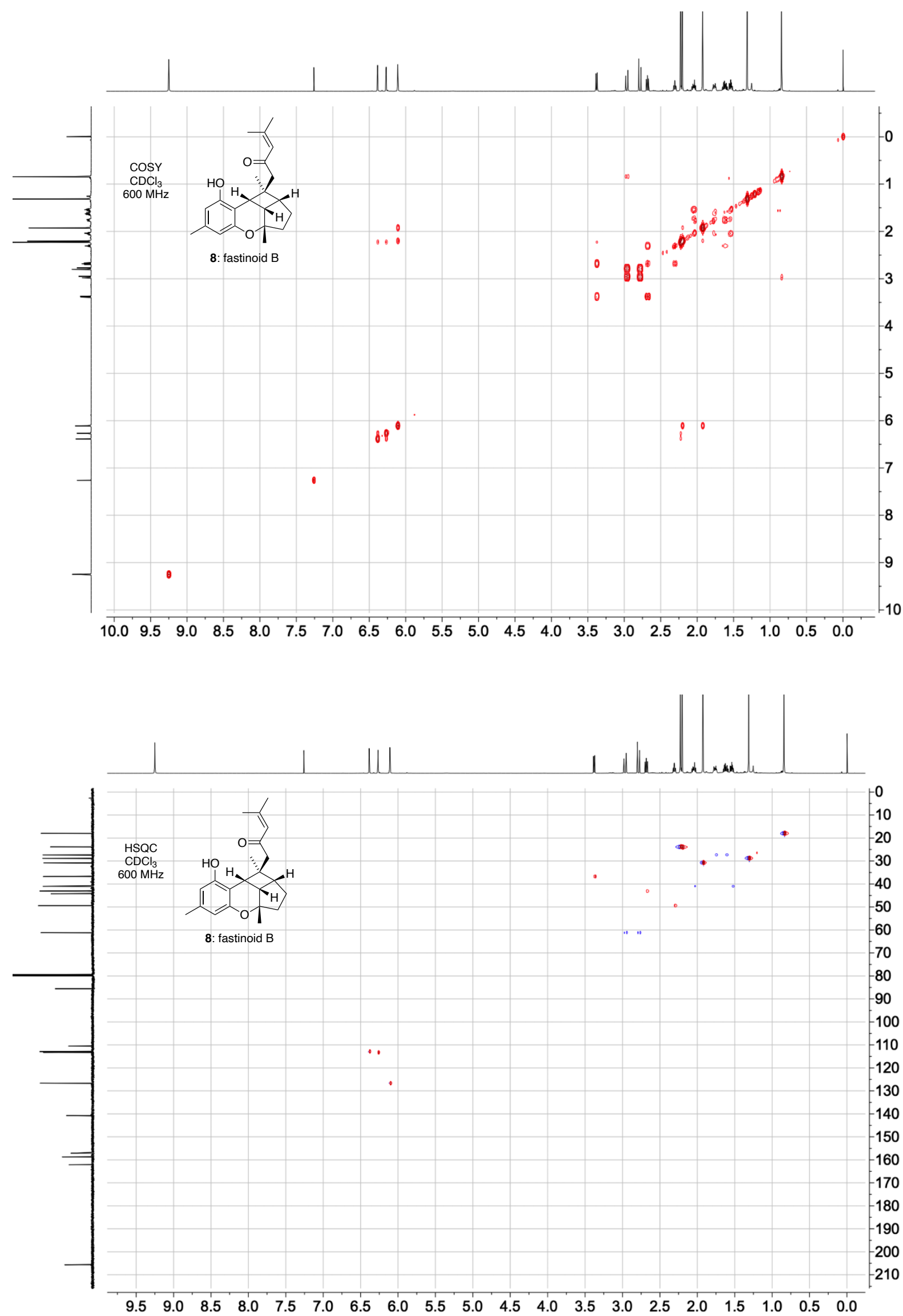

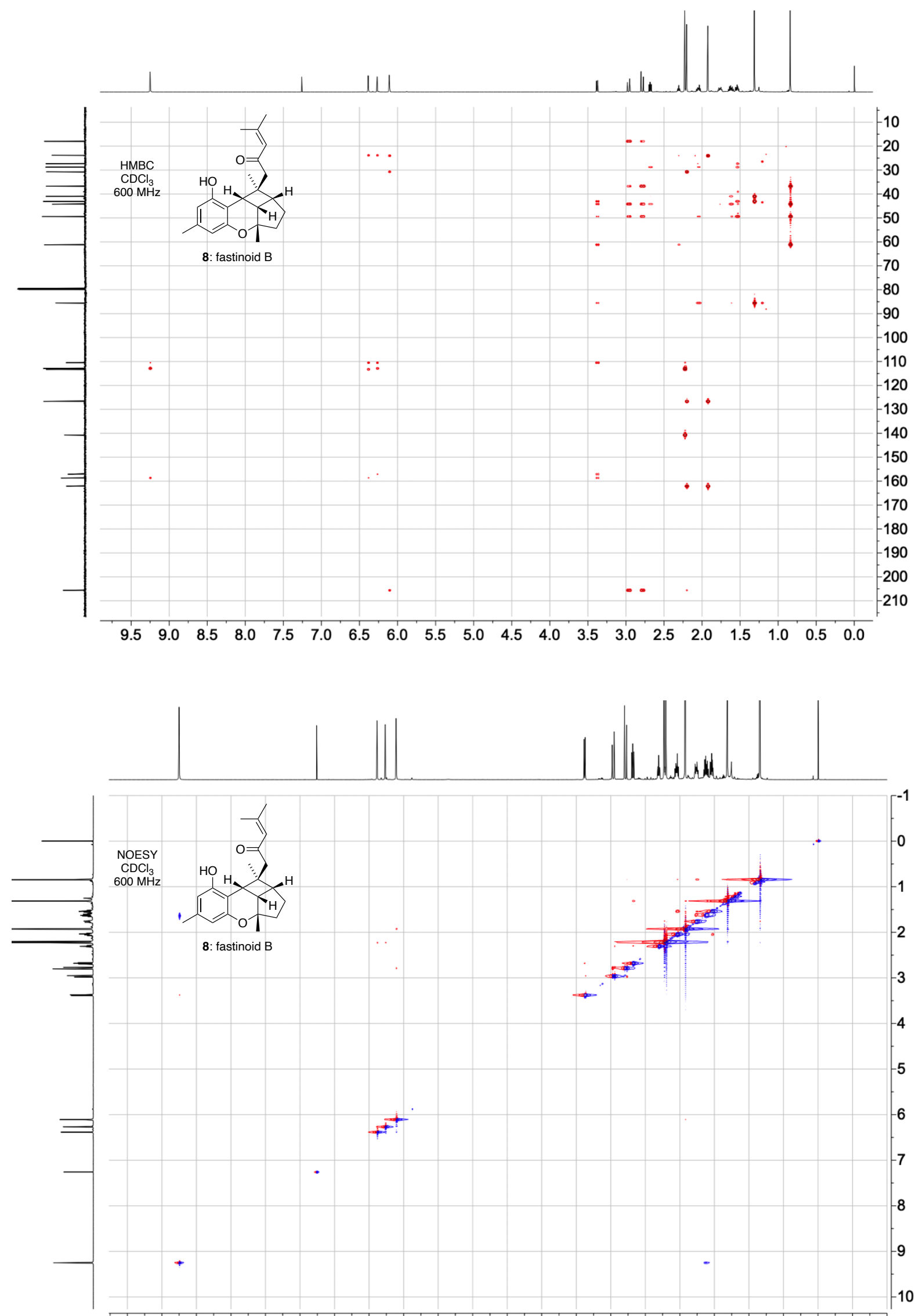

\begin{tabular}{llllllllllllllllllllllllllll}
\hline 10.0 & 9.5 & 9.0 & 8.5 & 8.0 & 7.5 & 7.0 & 6.5 & 6.0 & 5.5 & 5.0 & 4.5 & 4.0 & 3.5 & 3.0 & 2.5 & 2.0 & 1.5 & 1.0 & 0.5 & 0.0 & -0.5 & -1.0
\end{tabular} 


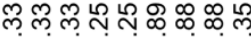

0

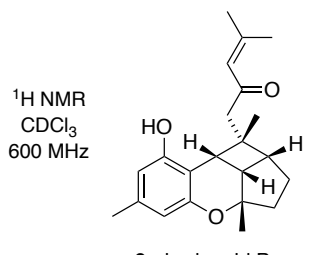

9: rhodonoid B

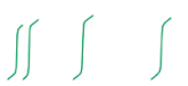

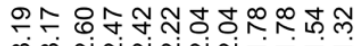

लु

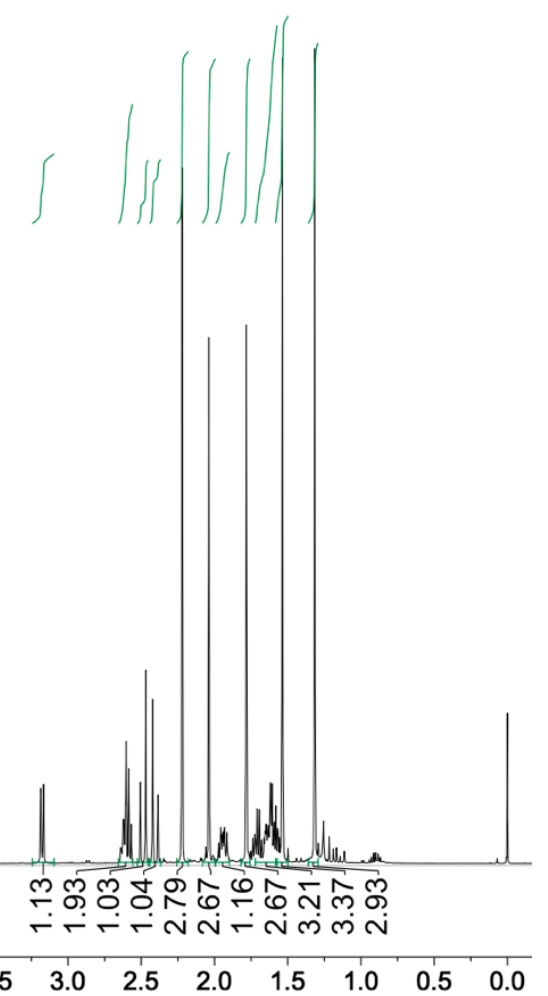

$\stackrel{0}{i}$

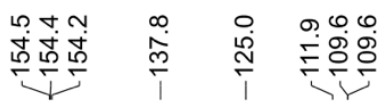

$\stackrel{\substack{p \\ \infty \\ 1}}{\infty}$

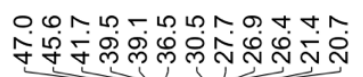

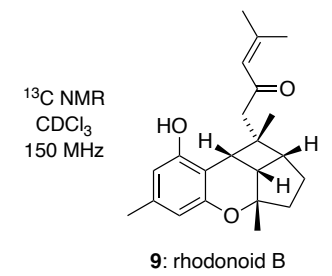

9: rhodonoid B
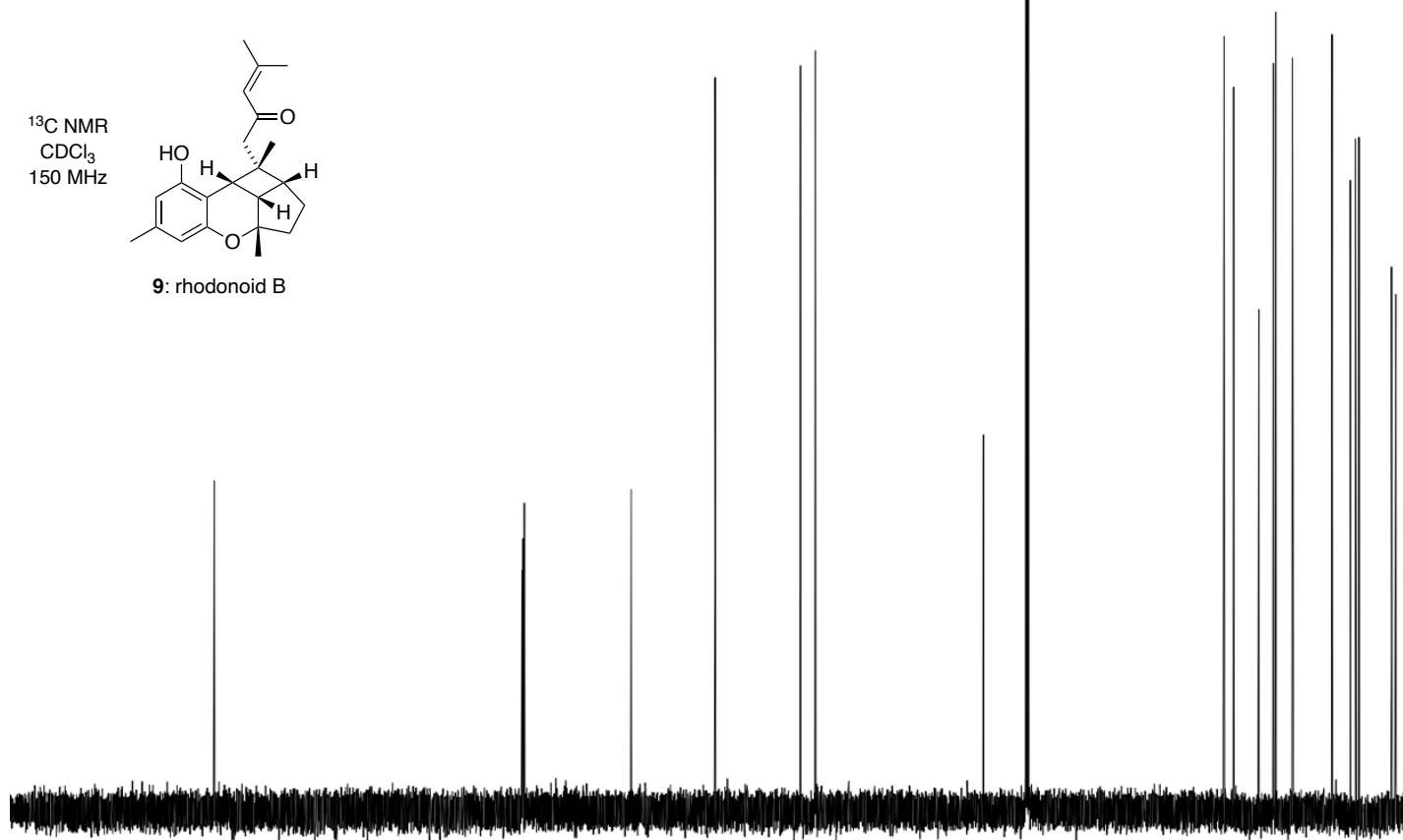

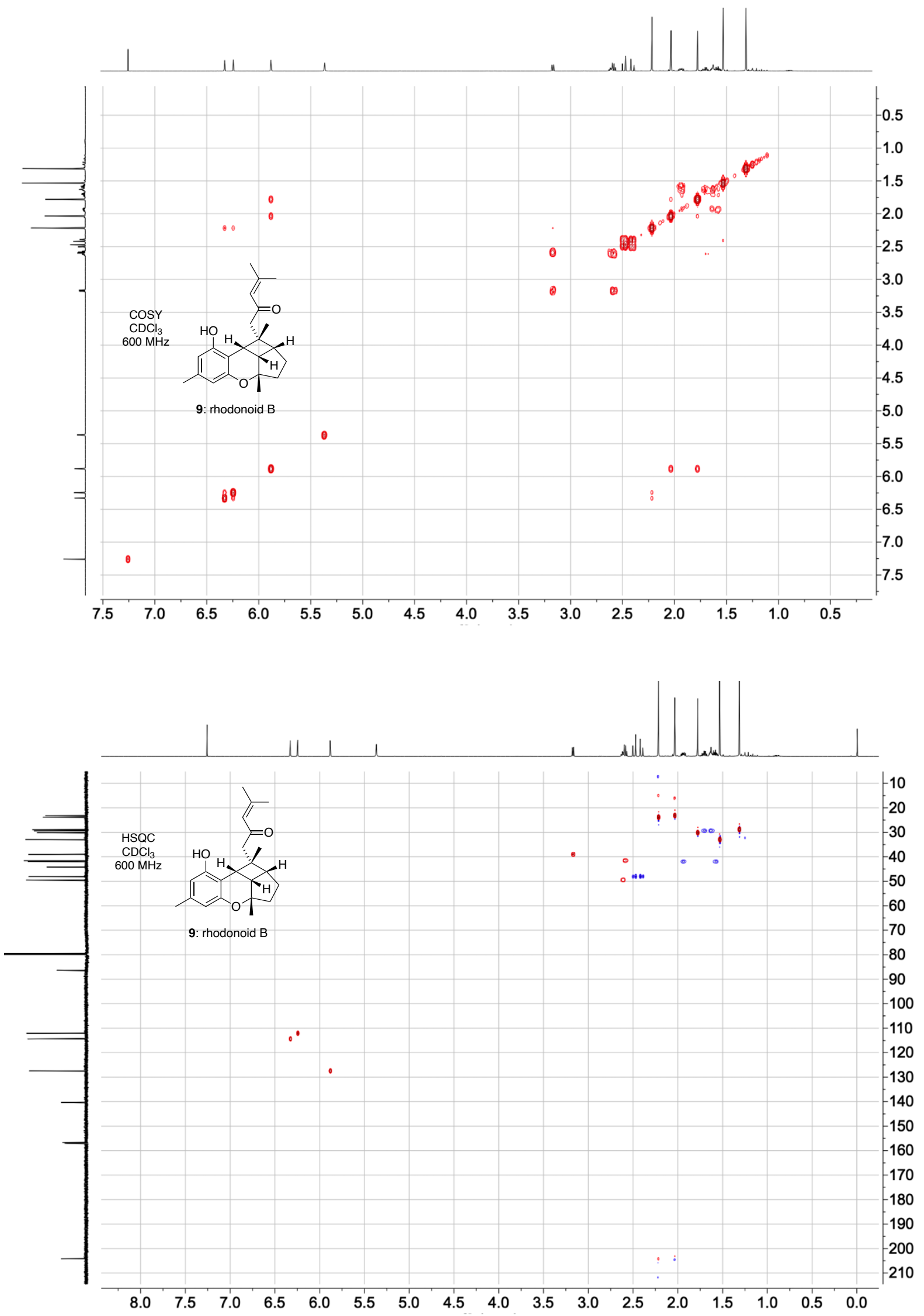

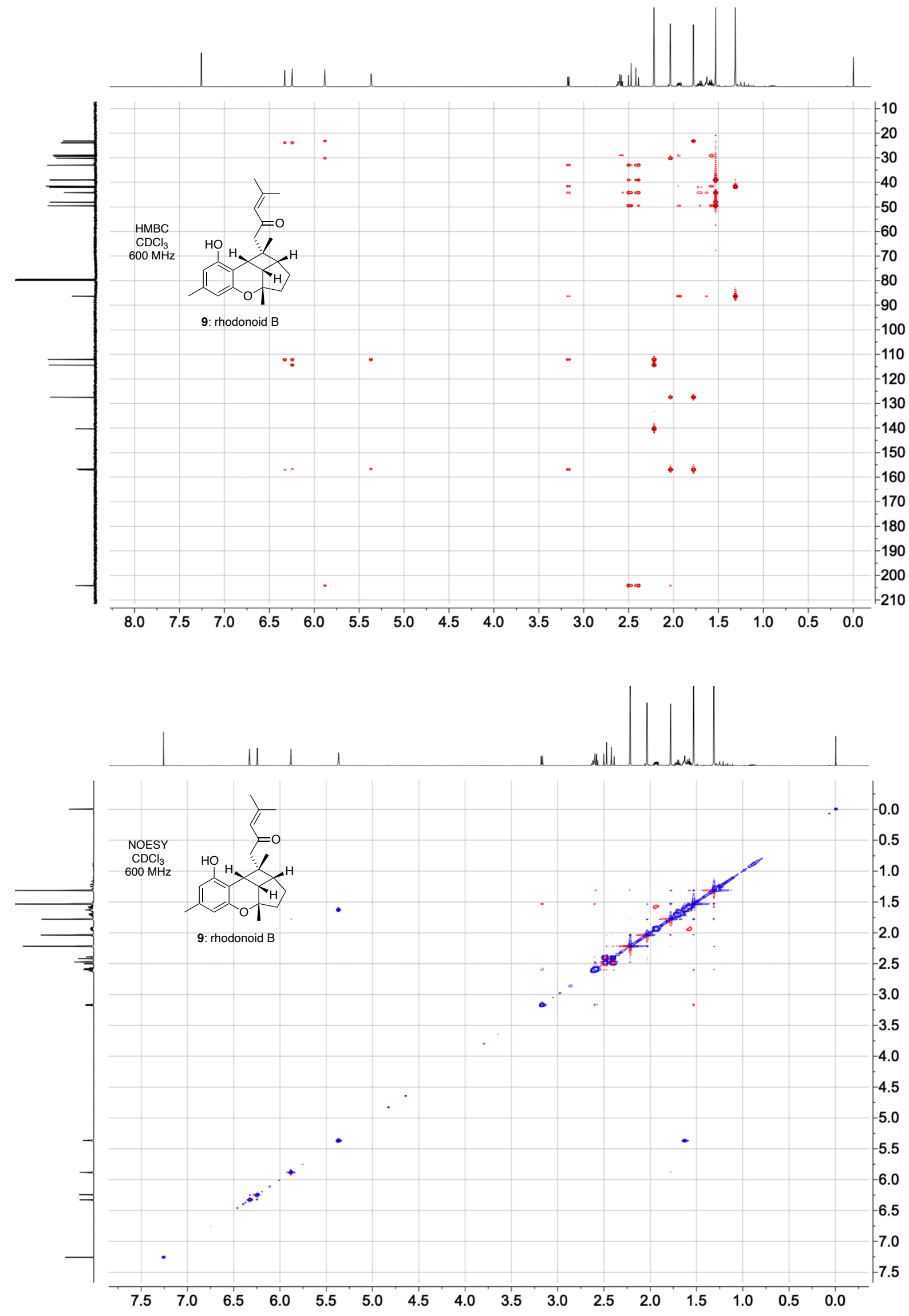


\section{Comparison of Natural and Synthetic NMR Data}

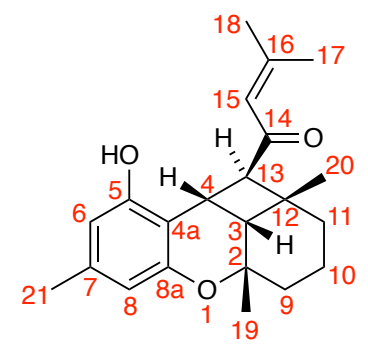

4: rubiginosin $A$

Table S1 - ${ }^{1} \mathrm{H}$ NMR and ${ }^{13} \mathrm{C}$ NMR Comparison for Rubiginosin A (4)

\begin{tabular}{|c|c|c|c|c|}
\hline \multirow[t]{2}{*}{$\begin{array}{c}\text { NMR } \\
\text { Assignment }\end{array}$} & \multicolumn{2}{|c|}{$\begin{array}{l}\text { Synthetic Sample }\left(\mathrm{CDCl}_{3}\right) \\
\text { George et al. (2020) }\end{array}$} & \multicolumn{2}{|c|}{$\begin{array}{l}\text { Natural Sample }\left(\mathrm{CDCl}_{3}\right) \\
\text { Yang et al. }(2018)^{4}\end{array}$} \\
\hline & $\begin{array}{l}{ }^{1} \mathrm{H} \text { NMR } \\
(600 \mathrm{MHz})\end{array}$ & $\begin{array}{c}{ }^{13} \text { C NMR } \\
(150 \text { MHz) }\end{array}$ & $\begin{array}{c}{ }^{1} \mathrm{H} \text { NMR } \\
(500 \mathrm{MHz})\end{array}$ & $\begin{array}{c}{ }^{13} \mathrm{C} \mathrm{NMR} \\
(125 \\
\text { MHz) }\end{array}$ \\
\hline 2 & -- & 75.3 & -- & 75.3 \\
\hline 3 & $\begin{array}{c}1.82 \\
(\mathrm{~d}, J=9.8 \mathrm{~Hz})\end{array}$ & 45.1 & $\begin{array}{c}1.82 \\
(\mathrm{~d}, J=9.8 \mathrm{~Hz})\end{array}$ & 45.1 \\
\hline 4 & $\begin{array}{c}3.82 \\
(\mathrm{t}, J=9.3 \mathrm{~Hz})\end{array}$ & 24.0 & $\begin{array}{c}3.83 \\
(\mathrm{t}, J=9.3 \mathrm{~Hz})\end{array}$ & 24.0 \\
\hline $4 a$ & -- & 112.1 & -- & 112.0 \\
\hline 5 & -- & 153.4 & -- & 154.4 \\
\hline $5-\mathrm{OH}$ & $7.48(\mathrm{~s})$ & -- & -- & -- \\
\hline 6 & $6.40(\mathrm{~s})$ & 110.6 & $6.31(\mathrm{~s})$ & 111.5 \\
\hline 7 & -- & 138.0 & -- & 137.9 \\
\hline 8 & $6.30(\mathrm{~s})$ & 111.5 & $6.41(\mathrm{~s})$ & 110.6 \\
\hline $8 \mathrm{a}$ & -- & 154.4 & -- & 153.4 \\
\hline 9 & $\begin{array}{c}1.37-1.24(\mathrm{~m}) \\
2.09 \\
(\mathrm{dt}, J=13.9,3.7 \mathrm{~Hz})\end{array}$ & 36.4 & $\begin{array}{l}1.37(\mathrm{~m}) \\
2.07(\mathrm{~m})\end{array}$ & 36.4 \\
\hline 10 & $\begin{array}{l}1.70-1.62(\mathrm{~m}) \\
2.01-1.93(\mathrm{~m})\end{array}$ & 17.4 & $\begin{array}{l}1.66(\mathrm{~m}) \\
1.97(\mathrm{~m})\end{array}$ & 17.4 \\
\hline 11 & $\begin{array}{l}1.37-1.24(\mathrm{~m}) \\
1.89-1.84(\mathrm{~m})\end{array}$ & 34.4 & $\begin{array}{l}1.30(\mathrm{~m}) \\
1.88(\mathrm{~m})\end{array}$ & 34.4 \\
\hline 12 & -- & 39.5 & -- & 39.4 \\
\hline 13 & $\begin{array}{c}2.96 \\
(\mathrm{~d}, J=9.3 \mathrm{~Hz})\end{array}$ & 58.0 & $\begin{array}{c}2.97 \\
(\mathrm{~d}, J=9.3 \mathrm{~Hz})\end{array}$ & 58.0 \\
\hline 14 & -- & 203.2 & -- & 203.2 \\
\hline 15 & $5.87(\mathrm{~m})$ & 122.4 & $5.88(\mathrm{~m})$ & 122.4 \\
\hline 16 & -- & 158.1 & -- & 158.0 \\
\hline 17 & $2.16(\mathrm{~s})$ & 21.5 & $2.17(\mathrm{~s})$ & 21.5 \\
\hline 18 & $1.90(\mathrm{~s})$ & 28.2 & $1.91(\mathrm{~s})$ & 28.2 \\
\hline 19 & $1.14(\mathrm{~s})$ & 29.7 & $1.14(\mathrm{~s})$ & 29.6 \\
\hline 20 & $1.15(\mathrm{~s})$ & 25.9 & $1.16(\mathrm{~s})$ & 25.8 \\
\hline 21 & $2.23(\mathrm{~s})$ & 21.6 & $2.23(\mathrm{~s})$ & 21.5 \\
\hline
\end{tabular}




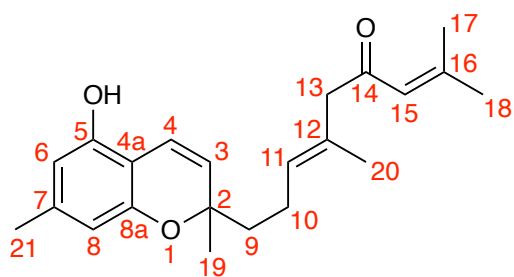

11: rubiginosin $\mathrm{E}$

Table S2 - ${ }^{1} \mathrm{H}$ NMR and ${ }^{13} \mathrm{C}$ NMR Comparison for Rubiginosin E (11)

\begin{tabular}{|c|c|c|c|c|}
\hline \multirow[t]{2}{*}{$\begin{array}{c}\text { NMR } \\
\text { Assignment }\end{array}$} & \multicolumn{2}{|c|}{$\begin{array}{c}\text { Synthetic Sample }\left(\mathrm{CD}_{3} \mathrm{OD}\right) \\
\text { George et al. (2020) }\end{array}$} & \multicolumn{2}{|c|}{$\begin{array}{c}\text { Natural Sample }\left(\mathrm{CD}_{3} \mathrm{OD}\right) \\
\text { Yang et al. }(2018)^{4}\end{array}$} \\
\hline & $\begin{array}{c}{ }^{1} \mathrm{H} \text { NMR } \\
(600 \mathrm{MHz})\end{array}$ & $\begin{array}{l}{ }^{13} \mathrm{C} \text { NMR } \\
(150 \mathrm{MHz})\end{array}$ & $\begin{array}{c}{ }^{1} \mathrm{H} \text { NMR } \\
(500 \mathrm{MHz})\end{array}$ & $\begin{array}{l}{ }^{13} \mathrm{C} \mathrm{NMR} \\
(125 \mathrm{MHz})\end{array}$ \\
\hline 2 & -- & 78.9 & -- & 78.9 \\
\hline 3 & $\begin{array}{c}5.46 \\
(\mathrm{~d}, J=10.0 \mathrm{~Hz})\end{array}$ & 127.1 & $\begin{array}{c}5.46 \\
(\mathrm{~d}, J=9.9 \mathrm{~Hz})\end{array}$ & 127.1 \\
\hline 4 & $\begin{array}{c}6.63 \\
(\mathrm{~d}, J=10.0 \mathrm{~Hz})\end{array}$ & 118.8 & $\begin{array}{c}6.63 \\
(\mathrm{~d}, J=9.9 \mathrm{~Hz})\end{array}$ & 118.8 \\
\hline $4 a$ & -- & 108.1 & -- & 109.2 \\
\hline 5 & -- & 155.2 & -- & 155.2 \\
\hline 6 & $6.08(\mathrm{~s})$ & 109.3 & $6.14(\mathrm{~s})$ & 109.4 \\
\hline 7 & -- & 140.4 & -- & 140.4 \\
\hline 8 & $6.14(\mathrm{~s})$ & 109.2 & $6.08(\mathrm{~s})$ & 109.2 \\
\hline $8 \mathrm{a}$ & -- & 154.2 & -- & 154.1 \\
\hline 9 & $1.73-1.64(\mathrm{~m})$ & 41.7 & $1.68(\mathrm{~m})$ & 41.7 \\
\hline 10 & $2.19-2.14(\mathrm{~m})$ & 23.9 & $2.16(\mathrm{~m})$ & 23.9 \\
\hline 11 & $\begin{array}{c}5.29 \\
(\mathrm{t}, J=7.2 \mathrm{~Hz})\end{array}$ & 130.6 & $5.29(\mathrm{~m})$ & 130.6 \\
\hline 12 & -- & 130.7 & -- & 130.7 \\
\hline 13 & $3.02(\mathrm{~s})$ & 56.0 & $3.02(\mathrm{~s})$ & 56.0 \\
\hline 14 & -- & 201.9 & -- & 201.9 \\
\hline 15 & 6.18 (br s) & 123.9 & $6.18(\mathrm{~m})$ & 123.9 \\
\hline 16 & -- & 157.8 & -- & 157.8 \\
\hline 17 & $2.11(\mathrm{~s})$ & 20.8 & $2.11(\mathrm{~s})$ & 20.8 \\
\hline 18 & $1.88(\mathrm{~s})$ & 27.7 & $1.88(\mathrm{~s})$ & 27.7 \\
\hline 19 & $1.33(\mathrm{~s})$ & 26.6 & $1.33(\mathrm{~s})$ & 26.6 \\
\hline 20 & $1.56(\mathrm{~s})$ & 16.3 & $1.56(\mathrm{~s})$ & 16.5 \\
\hline 21 & $2.16(\mathrm{~s})$ & 21.7 & $2.16(\mathrm{~s})$ & 21.7 \\
\hline
\end{tabular}




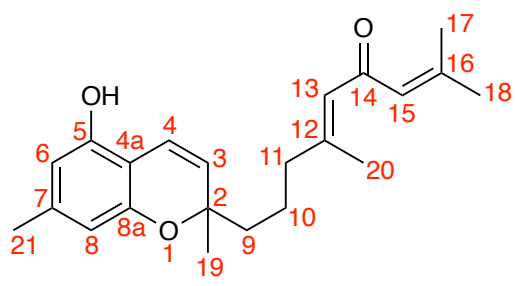

12: rubiginosin $D$

Table S3 - ${ }^{1} \mathrm{H}$ NMR and ${ }^{13} \mathrm{C}$ NMR Comparison for Rubiginosin D (12)

\begin{tabular}{|c|c|c|c|c|}
\hline \multirow[t]{2}{*}{$\begin{array}{c}\text { NMR } \\
\text { Assignment }\end{array}$} & \multicolumn{2}{|c|}{$\begin{array}{c}\text { Synthetic Sample }\left(\mathrm{CD}_{3} \mathrm{OD}\right) \\
\text { George et al. }(2020)\end{array}$} & \multicolumn{2}{|c|}{$\begin{array}{c}\text { Natural Sample }\left(\mathrm{CD}_{3} \mathrm{OD}\right) \\
\text { Yang et al. }(2018)^{4}\end{array}$} \\
\hline & $\begin{array}{l}{ }^{1} \mathrm{H} \text { NMR } \\
(600 \mathrm{MHz})\end{array}$ & $\begin{array}{l}{ }^{13} \mathrm{C} \text { NMR } \\
(150 \mathrm{MHz})\end{array}$ & $\begin{array}{c}{ }^{1} \mathrm{H} \text { NMR } \\
(500 \mathrm{MHz})\end{array}$ & $\begin{array}{c}{ }^{13} \mathrm{C} \text { NMR } \\
(125 \mathrm{MHz})\end{array}$ \\
\hline 2 & -- & 78.9 & -- & 78.9 \\
\hline 3 & $\begin{array}{c}5.45 \\
(\mathrm{~d}, J=10.0 \mathrm{~Hz})\end{array}$ & 127.1 & $5.45(\mathrm{~d}, J=9.8 \mathrm{~Hz})$ & 127.1 \\
\hline 4 & $\begin{array}{c}6.64 \\
(\mathrm{dd}, J=10.0,0.7 \mathrm{~Hz})\end{array}$ & 118.9 & $6.64(\mathrm{~d}, J=9.8 \mathrm{~Hz})$ & 118.8 \\
\hline $4 a$ & -- & 108.1 & -- & 108.1 \\
\hline 5 & -- & 155.2 & -- & 158.2 \\
\hline 6 & $6.07(\mathrm{~s})$ & 109.2 & $6.14(\mathrm{~s})$ & 109.3 \\
\hline 7 & -- & 140.4 & -- & 140.4 \\
\hline 8 & $6.14(\mathrm{~s})$ & 109.3 & $6.08(\mathrm{~s})$ & 109.3 \\
\hline $8 \mathrm{a}$ & -- & 154.2 & -- & 154.2 \\
\hline 9 & $1.63-1.62(\mathrm{~m})$ & 41.4 & $1.63(\mathrm{~m})$ & 41.4 \\
\hline 10 & $1.63-1.62(\mathrm{~m})$ & 22.9 & $1.63(\mathrm{~m})$ & 22.9 \\
\hline 11 & $2.16-2.13(\mathrm{~m})$ & 42.2 & $2.16(\mathrm{~m})$ & 42.2 \\
\hline 12 & -- & 159.5 & -- & 159.5 \\
\hline 13 & $6.06-6.05(\mathrm{~m})$ & 126.8 & $6.09(\mathrm{~m})$ & 126.8 \\
\hline 14 & -- & 193.8 & -- & 193.9 \\
\hline 15 & $6.10-6.09(\mathrm{~m})$ & 127.3 & $6.10(\mathrm{~m})$ & 127.3 \\
\hline 16 & -- & 156.2 & -- & 155.2 \\
\hline 17 & $2.12(\mathrm{~s})$ & 20.8 & $2.12(\mathrm{~s})$ & 20.7 \\
\hline 18 & $1.90(\mathrm{~s})$ & 27.8 & $1.90(\mathrm{~s})$ & 27.7 \\
\hline 19 & $1.32(\mathrm{~s})$ & 26.6 & $1.33(\mathrm{~s})$ & 26.6 \\
\hline 20 & $2.08(\mathrm{~s})$ & 19.3 & $2.08(\mathrm{~s})$ & 19.3 \\
\hline 21 & $2.16(\mathrm{~s})$ & 21.7 & $2.16(\mathrm{~s})$ & 21.7 \\
\hline
\end{tabular}




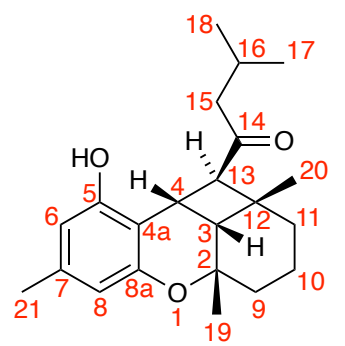

2: rubiginosin $G$

Table S4 - ${ }^{1} \mathrm{H}$ NMR and ${ }^{13} \mathrm{C}$ NMR Comparison for Rubiginosin G (2)

\begin{tabular}{|c|c|c|c|c|}
\hline \multirow[t]{2}{*}{$\begin{array}{c}\text { NMR } \\
\text { Assignment }\end{array}$} & \multicolumn{2}{|c|}{$\begin{array}{c}\text { Synthetic Sample }\left(\mathrm{CDCl}_{3}\right) \\
\text { George et al. }(\mathbf{2 0 2 0})\end{array}$} & \multicolumn{2}{|c|}{$\begin{array}{l}\text { Natural Sample }\left(\mathrm{CDCl}_{3}\right) \\
\text { Yang et al. }(2018)^{4}\end{array}$} \\
\hline & $\begin{array}{c}{ }^{1} \mathrm{H} \text { NMR } \\
(600 \mathrm{MHz})\end{array}$ & $\begin{array}{c}{ }^{13} \mathrm{C} \mathrm{NMR} \\
(\mathbf{1 5 0} \\
\mathrm{MHz})\end{array}$ & $\begin{array}{c}{ }^{1} \mathrm{H} \text { NMR } \\
(500 \mathrm{MHz})\end{array}$ & $\begin{array}{c}{ }^{13} \mathrm{C} \mathrm{NMR} \\
(125 \\
\mathrm{MHz})\end{array}$ \\
\hline 2 & -- & 75.3 & -- & 75.3 \\
\hline 3 & $1.80(\mathrm{~d}, J=9.3 \mathrm{~Hz})$ & 45.2 & $1.79(\mathrm{~d}, J=9.5 \mathrm{~Hz})$ & 45.2 \\
\hline 4 & $3.81(\mathrm{t}, J=9.4 \mathrm{~Hz})$ & 23.9 & $3.81(\mathrm{t}, J=9.5 \mathrm{~Hz})$ & 23.9 \\
\hline $4 a$ & -- & 111.8 & -- & 111.9 \\
\hline 5 & -- & 153.1 & -- & 154.6 \\
\hline $5-\mathrm{OH}$ & $6.83(\mathrm{~s})$ & -- & -- & -- \\
\hline 6 & $6.40(\mathrm{~s})$ & 110.6 & $6.30(\mathrm{~s})$ & 111.8 \\
\hline 7 & -- & 138.1 & -- & 138.2 \\
\hline 8 & $6.31(\mathrm{~s})$ & 111.7 & $6.40(\mathrm{~s})$ & 110.6 \\
\hline $8 \mathrm{a}$ & -- & 154.5 & -- & 153.2 \\
\hline 9 & $\begin{array}{l}1.37-1.33(\mathrm{~m}) \\
2.09-2.05(\mathrm{~m})\end{array}$ & 36.6 & $\begin{array}{l}1.34(\mathrm{~m}) \\
2.08(\mathrm{~m})\end{array}$ & 36.6 \\
\hline 10 & $\begin{array}{l}1.70-1.63(\mathrm{~m}) \\
2.00-1.91(\mathrm{~m})\end{array}$ & 17.3 & $\begin{array}{l}1.65(\mathrm{~m}) \\
1.95(\mathrm{~m}) \\
\end{array}$ & 17.3 \\
\hline 11 & $\begin{array}{l}1.33-1.28(\mathrm{~m}) \\
1.87-1.81(\mathrm{~m})\end{array}$ & 34.5 & $\begin{array}{l}1.30(\mathrm{~m}) \\
1.84(\mathrm{~m})\end{array}$ & 34.5 \\
\hline 12 & -- & 39.4 & -- & 39.5 \\
\hline 13 & $2.91(\mathrm{~d}, J=9.4 \mathrm{~Hz})$ & 57.4 & $2.91(\mathrm{~d}, J=9.8 \mathrm{~Hz})$ & 57.4 \\
\hline 14 & -- & 213.9 & -- & 213.9 \\
\hline 15 & $\begin{array}{c}2.18 \\
(\mathrm{dd}, J=13.5,6.6 \mathrm{~Hz})\end{array}$ & 50.9 & $2.17(\mathrm{~m})$ & 50.9 \\
\hline 16 & $2.15-2.10(\mathrm{~m})$ & 24.2 & $2.11(\mathrm{~m})$ & 24.3 \\
\hline 17 & $0.89(\mathrm{~d}, J=6.4 \mathrm{~Hz})$ & 23.0 & $0.89(\mathrm{~d}, J=6.7 \mathrm{~Hz})$ & 23.0 \\
\hline 18 & $0.86(\mathrm{~d}, J=6.4 \mathrm{~Hz})$ & 22.8 & $0.86(\mathrm{~d}, J=6.7 \mathrm{~Hz})$ & 22.8 \\
\hline 19 & $1.11(\mathrm{~s})$ & 29.6 & $1.11(\mathrm{~s})$ & 29.6 \\
\hline 20 & $1.17(\mathrm{~s})$ & 25.8 & $1.17(\mathrm{~s})$ & 25.9 \\
\hline 21 & $2.23(\mathrm{~s})$ & 21.6 & $2.23(\mathrm{~s})$ & 21.6 \\
\hline
\end{tabular}




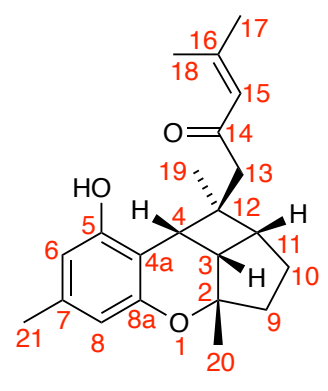

8: fastinoid $B$

Table S5 - ${ }^{1} \mathrm{H}$ NMR and ${ }^{13} \mathrm{C}$ NMR Comparison for Fastinoid B (8)

\begin{tabular}{|c|c|c|c|c|}
\hline \multirow[t]{2}{*}{$\begin{array}{c}\text { NMR } \\
\text { Assignment }\end{array}$} & \multicolumn{2}{|c|}{$\begin{array}{c}\text { Synthetic Sample }\left(\mathrm{CDCl}_{3}\right) \\
\text { George et al. }(2020)\end{array}$} & \multicolumn{2}{|c|}{$\begin{array}{c}\text { Natural Sample }\left(\mathrm{CDCl}_{3}\right) \\
\text { Hou et al. }(2019)^{5} \\
\end{array}$} \\
\hline & $\begin{array}{c}{ }^{1} \mathrm{H} \text { NMR } \\
(600 \mathrm{MHz})\end{array}$ & $\begin{array}{l}{ }^{13} \mathrm{C} \text { NMR } \\
(150 \mathrm{MHz})\end{array}$ & $\begin{array}{l}{ }^{1} \mathrm{H} \text { NMR } \\
(500 \mathrm{MHz})\end{array}$ & $\begin{array}{l}{ }^{13} \mathrm{C} \text { NMR } \\
(125 \mathrm{MHz})\end{array}$ \\
\hline 2 & -- & 83.0 & -- & 83.0 \\
\hline 3 & $2.68(\mathrm{t}, J=8.8 \mathrm{~Hz})$ & 40.6 & $2.68(\mathrm{t}, J=8.8 \mathrm{~Hz})$ & 40.6 \\
\hline 4 & $3.38(\mathrm{~d}, J=9.6 \mathrm{~Hz})$ & 34.2 & $3.38(\mathrm{~d}, J=10.0 \mathrm{~Hz})$ & 34.3 \\
\hline $4 \mathrm{a}$ & -- & 108.0 & -- & 108.0 \\
\hline 5 & -- & 156.2 & - & 156.2 \\
\hline $5-\mathrm{OH}$ & $9.25(\mathrm{~s})$ & -- & -- & -- \\
\hline 6 & $6.39(\mathrm{~s})$ & 110.4 & 6.39 (br s) & 110.4 \\
\hline 7 & -- & 138.2 & -- & 138.2 \\
\hline 8 & $6.27(\mathrm{~s})$ & 110.8 & 6.27 (br s) & 110.8 \\
\hline $8 \mathrm{a}$ & -- & 154.6 & -- & 154.6 \\
\hline 9 & $\begin{array}{l}2.08-2.02(\mathrm{~m}) \\
1.56-1.51(\mathrm{~m})\end{array}$ & 38.5 & $\begin{array}{l}2.04(\mathrm{~m}) \\
1.54(\mathrm{~m})\end{array}$ & 38.6 \\
\hline 10 & $\begin{array}{l}1.80-1.73(\mathrm{~m}) \\
1.66-1.59(\mathrm{~m}) \\
\end{array}$ & 24.8 & $\begin{array}{l}1.76(\mathrm{~m}) \\
1.63(\mathrm{~m}) \\
\end{array}$ & 24.8 \\
\hline 11 & $\begin{array}{c}2.31 \\
(\mathrm{td}, J=8.4,2.9 \mathrm{~Hz})\end{array}$ & 46.9 & $\begin{array}{c}2.31 \\
(\mathrm{td}, J=8.4,2.4 \mathrm{~Hz})\end{array}$ & 47.0 \\
\hline 12 & -- & 41.7 & -- & 41.8 \\
\hline 13 & $\begin{array}{l}2.97(\mathrm{~d}, J=17.7 \mathrm{~Hz}) \\
2.79(\mathrm{~d}, J=17.7 \mathrm{~Hz})\end{array}$ & 58.7 & $\begin{array}{l}2.97(\mathrm{~d}, J=17.6 \mathrm{~Hz}) \\
2.79(\mathrm{~d}, J=17.6 \mathrm{~Hz})\end{array}$ & 58.7 \\
\hline 14 & -- & 203.1 & -- & 203.1 \\
\hline 15 & $6.11(\mathrm{~s})$ & 124.2 & 6.11 (br s) & 124.2 \\
\hline 16 & -- & 159.6 & -- & 159.6 \\
\hline 17 & $1.92(\mathrm{~s})$ & 28.2 & 1.93 (br s) & 28.2 \\
\hline 18 & $2.20(\mathrm{~s})$ & 21.5 & 2.21 (br s) & 21.5 \\
\hline 19 & $0.84(\mathrm{~s})$ & 15.5 & $0.84(\mathrm{~s})$ & 15.5 \\
\hline 20 & $1.31(\mathrm{~s})$ & 26.3 & $1.32(\mathrm{~s})$ & 26.3 \\
\hline 21 & $2.23(\mathrm{~s})$ & 21.3 & $2.23(\mathrm{~s})$ & 21.3 \\
\hline
\end{tabular}




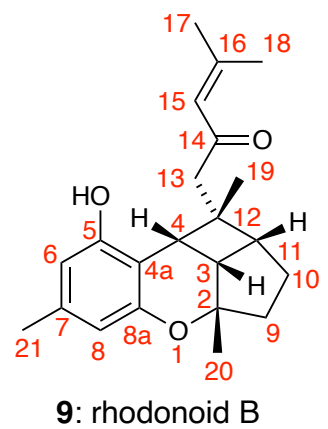

Table S6 - ${ }^{1} \mathrm{H}$ NMR and ${ }^{13} \mathrm{C}$ NMR Comparison for Rhodonoid B (9)

\begin{tabular}{|c|c|c|c|c|}
\hline \multirow[t]{2}{*}{$\begin{array}{c}\text { NMR } \\
\text { Assignment }\end{array}$} & \multicolumn{2}{|c|}{$\begin{array}{c}\text { Synthetic Sample }\left(\mathrm{CDCl}_{3}\right) \\
\text { George et al. (2020) }\end{array}$} & \multicolumn{2}{|c|}{$\begin{array}{l}\text { Natural Sample }\left(\mathrm{CDCl}_{3}\right) \\
\text { Hou et al. }(2015)^{6}\end{array}$} \\
\hline & $\begin{array}{c}{ }^{1} \mathrm{H} \text { NMR } \\
(600 \mathrm{MHz})\end{array}$ & $\begin{array}{l}{ }^{13} \mathrm{C} \text { NMR } \\
(150 \mathrm{MHz})\end{array}$ & $\begin{array}{l}{ }^{1} \text { H NMR } \\
(600 \mathrm{MHz})\end{array}$ & $\begin{array}{l}{ }^{13} \mathrm{C} \mathrm{NMR} \\
(150 \mathrm{MHz})\end{array}$ \\
\hline 2 & -- & 83.9 & -- & 83.9 \\
\hline 3 & $\begin{array}{c}2.60 \\
(\mathrm{dd}, J=9.0,8.0 \mathrm{~Hz})\end{array}$ & 39.1 & $\begin{array}{c}2.59 \\
(\mathrm{dd}, J=9.0,8.0 \mathrm{~Hz})\end{array}$ & 39.1 \\
\hline 4 & $\begin{array}{c}3.18 \\
(\mathrm{~d}, J=9.3 \mathrm{~Hz})\end{array}$ & 36.5 & $\begin{array}{c}3.17 \\
(\mathrm{~d}, J=9.0 \mathrm{~Hz})\end{array}$ & 36.6 \\
\hline $4 a$ & -- & 109.6 & -- & 109.6 \\
\hline 5 & -- & 154.2 & -- & 154.2 \\
\hline $5-\mathrm{OH}$ & 5.35 (br s) & -- & 5.42 (br s) & -- \\
\hline 6 & $6.25(\mathrm{~s})$ & 109.6 & 6.25 (br s) & 109.6 \\
\hline 7 & -- & 137.8 & -- & 137.8 \\
\hline 8 & $6.33(\mathrm{~s})$ & 111.9 & 6.33 (br s) & 111.9 \\
\hline $8 \mathrm{a}$ & -- & 154.5 & -- & 154.6 \\
\hline 9 & $\begin{array}{l}1.60-1.56(\mathrm{~m}) \\
1.98-1.90(\mathrm{~m})\end{array}$ & 39.5 & $\begin{array}{l}1.94(\mathrm{~m}) \\
1.58(\mathrm{~m})\end{array}$ & 39.5 \\
\hline 10 & $\begin{array}{c}1.66-1.61(\mathrm{~m}) \\
1.75-1.68(\mathrm{~m}, 1 \mathrm{H})\end{array}$ & 26.9 & $\begin{array}{l}1.72(\mathrm{~m}) \\
1.63(\mathrm{~m})\end{array}$ & 26.9 \\
\hline 11 & $\begin{array}{c}2.62 \\
(\mathrm{td}, J=8.0,2.6 \mathrm{~Hz})\end{array}$ & 47.0 & $2.62(\mathrm{~m})$ & 47.0 \\
\hline 12 & -- & 41.7 & -- & 41.7 \\
\hline 13 & $\begin{array}{l}2.42(\mathrm{~d}, J=18.3 \mathrm{~Hz}) \\
2.47(\mathrm{~d}, J=18.3 \mathrm{~Hz})\end{array}$ & 45.6 & $\begin{array}{l}2.41(\mathrm{~d}, J=18.6 \mathrm{~Hz}) \\
2.49(\mathrm{~d}, J=18.6 \mathrm{~Hz})\end{array}$ & 45.6 \\
\hline 14 & -- & 201.6 & -- & 201.6 \\
\hline 15 & $5.89-5.88(\mathrm{~m})$ & 125.0 & 5.89 (br s) & 125.0 \\
\hline 16 & -- & 154.4 & -- & 154.4 \\
\hline 17 & $1.78(\mathrm{~s})$ & 27.7 & 1.78 (br s) & 27.7 \\
\hline 18 & $2.04(\mathrm{~s})$ & 20.7 & 2.04 (br s) & 20.7 \\
\hline 19 & $1.54(\mathrm{~s})$ & 30.5 & $1.53(\mathrm{~s})$ & 30.5 \\
\hline 20 & $1.32(\mathrm{~s})$ & 26.4 & $1.32(\mathrm{~s})$ & 26.4 \\
\hline 21 & $2.22(\mathrm{~s})$ & 21.4 & $2.22(\mathrm{~s})$ & 21.4 \\
\hline
\end{tabular}




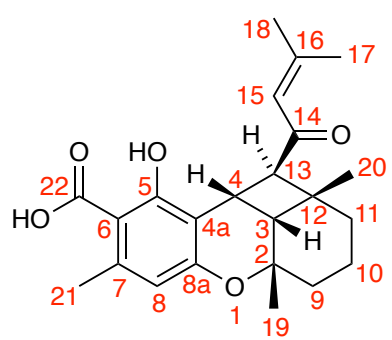

5: rubiginosin $\mathrm{B}$

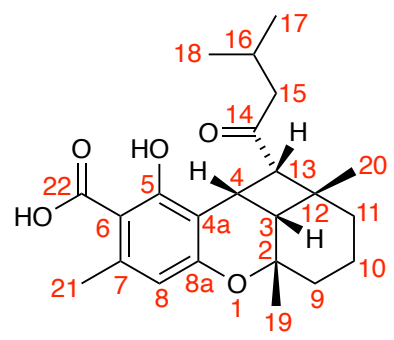

3: anthopogochromane originally proposed structure

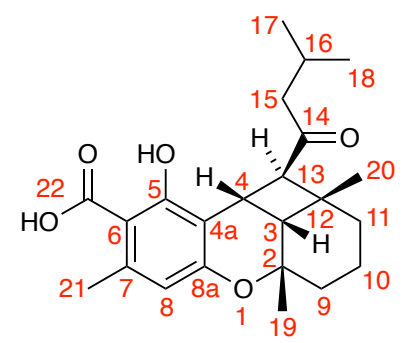

28: anthopogochromane revised structure

Table S7 - ${ }^{1} \mathrm{H}$ NMR and ${ }^{13} \mathrm{C}$ NMR comparison of Rubiginosin B (5) and Anthopogochromane

\begin{tabular}{|c|c|c|c|c|}
\hline \multirow[t]{2}{*}{$\begin{array}{c}\text { NMR } \\
\text { Assignment }\end{array}$} & \multicolumn{2}{|c|}{$\begin{array}{l}\text { Rubiginosin } \mathrm{B}\left(\mathrm{CDCl}_{3}\right) \\
\quad \text { Yang et al. }(2018)^{4}\end{array}$} & \multicolumn{2}{|c|}{$\begin{array}{l}\text { Anthopogochromane }\left(\mathrm{CDCl}_{3}\right) \\
\text { Kitanaka et al. }(\mathbf{2 0 1 0})^{7}\end{array}$} \\
\hline & $\begin{array}{l}{ }^{1} \mathrm{H} \text { NMR } \\
(600 \mathrm{MHz}) \\
\end{array}$ & $\begin{array}{c}{ }^{13} \mathrm{C} \text { NMR } \\
(150 \mathrm{MHz})\end{array}$ & $\begin{array}{c}{ }^{1} \mathrm{H} \text { NMR } \\
(500 \mathrm{MHz}) \\
\end{array}$ & $\begin{array}{c}{ }^{13} \mathrm{C} \text { NMR } \\
(125 \mathrm{MHz}) \\
\end{array}$ \\
\hline 2 & -- & 77.4 & -- & 77.2 \\
\hline 3 & $1.89(\mathrm{~d}, J=9.8 \mathrm{~Hz})$ & 46.5 & $\begin{array}{c}1.88 \\
(\mathrm{~d}, J=9.1 \mathrm{~Hz})\end{array}$ & 46.3 \\
\hline 4 & $\begin{array}{c}4.27 \\
(\mathrm{dd}, J=9.8,8.9 \mathrm{~Hz})\end{array}$ & 24.0 & $\begin{array}{c}4.24 \\
(\mathrm{dd}, J=9.1,8.5 \mathrm{~Hz})\end{array}$ & 23.8 \\
\hline $4 \mathrm{a}$ & -- & 114.0 & -- & 113.3 \\
\hline 5 & -- & 161.1 & -- & 160.3 \\
\hline 6 & -- & 105.2 & -- & 104.7 \\
\hline 7 & -- & 142.5 & -- & 142.3 \\
\hline 8 & $6.35 \mathrm{~s}$ & 114.8 & $6.33(\mathrm{~s})$ & 114.6 \\
\hline $8 \mathrm{a}$ & -- & 160.7 & -- & 160.9 \\
\hline 9 & $\begin{array}{l}1.35(\mathrm{~m}) \\
2.08(\mathrm{~m}) \\
\end{array}$ & 36.3 & $2.15(\mathrm{~m})$ & 36.2 \\
\hline 10 & $\begin{array}{l}1.64(\mathrm{~m}) \\
1.98(\mathrm{~m})\end{array}$ & 17.2 & $1.38(\mathrm{~m})$ & 17.2 \\
\hline 11 & $\begin{array}{l}1.25(\mathrm{~m}) \\
1.84(\mathrm{~m})\end{array}$ & 34.1 & $1.61(\mathrm{~m})$ & 34.0 \\
\hline 12 & -- & 39.0 & -- & 38.8 \\
\hline 13 & $3.01(\mathrm{~d}, J=8.9 \mathrm{~Hz})$ & 57.5 & $3.00(\mathrm{~d}, J=8.5 \mathrm{~Hz})$ & 56.4 \\
\hline 14 & -- & 200.2 & -- & 210.2 \\
\hline 15 & $5.97(\mathrm{~s})$ & 123.5 & $2.25(\mathrm{~m})$ & 51.9 \\
\hline 16 & -- & 155.9 & $2.13(\mathrm{~m})$ & 24.1 \\
\hline 17 & $2.14(\mathrm{~s})$ & 21.1 & $0.84(\mathrm{~d}, J=6.2 \mathrm{~Hz})$ & 22.8 \\
\hline 18 & $1.85(\mathrm{~s})$ & 28.0 & $0.84(\mathrm{~d}, J=6.2 \mathrm{~Hz})$ & 22.8 \\
\hline 19 & $1.06(\mathrm{~s})$ & 29.0 & $1.05(\mathrm{~s})$ & 29.0 \\
\hline 20 & $1.12(\mathrm{~s})$ & 25.5 & $1.13(\mathrm{~s})$ & 25.4 \\
\hline 21 & $2.54(\mathrm{~s})$ & 24.3 & $2.52(\mathrm{~s})$ & 24.4 \\
\hline $22(\mathrm{COOH})$ & -- & 175.1 & $11.75(\mathrm{~s})$ & 174.8 \\
\hline
\end{tabular}

${ }^{7}$ Iwata, N.; Kitanaka, S. J. Nat. Prod. 2010, 73, 1203. 